TRANSACTIONS OF THE

AMERICAN MATHEMATICAL SOCIETY

Volume 362, Number 3, March 2010, Pages 1505-1546

S 0002-9947(09)04884-3

Article electronically published on October 20, 2009

\title{
THE REGULAR ALGEBRA OF A POSET
}

\author{
PERE ARA
}

\begin{abstract}
Let $K$ be a fixed field. We attach to each finite poset $\mathbb{P}$ a von Neumann regular $K$-algebra $Q_{K}(\mathbb{P})$ in a functorial way. We show that the monoid of isomorphism classes of finitely generated projective $Q_{K}(\mathbb{P})$-modules is the abelian monoid generated by $\mathbb{P}$ with the only relations given by $p=p+q$ whenever $q<p$ in $\mathbb{P}$. This extends the class of monoids for which there is a positive solution to the realization problem for von Neumann regular rings.
\end{abstract}

\section{INTRODUCTION}

An old theorem of Jacobson [27] states that, given a field $K$, the algebra $A=$ $K\langle x, y: y x=1\rangle$ has the following uniqueness property: Given any other $K$ algebra $R$ with elements $a, b$ such that $b a=1$ and $a b \neq 1$, the unique $K$-algebra homomorphism $A \rightarrow R$ sending $x$ to $a$ and $y$ to $b$ is one-to-one. A very natural representation of $A$ is the one given by the algebraic analogue of the Toeplitz algebra. This is defined as the subalgebra of $E=\operatorname{End}_{K}(K[z])$, generated by the unilateral shift $b$, given by multiplication by $z$, and the endomorphism $a \in E$ defined by $\left(z^{i}\right) a=z^{i-1}$ for $i \geq 1$ and (1) $a=0$. (Note that here endomorphisms act on the right of their arguments.) Clearly, $b a=1$, but $1-a b$ is the projection onto the one-dimensional subspace $1 \cdot K$ of $K[z]$, with kernel $z K[z]$.

Let $\psi: A \rightarrow E$ be the unique $K$-algebra homomorphism sending $x$ to $a$ and $y$ to $b$. For any polynomial $f \in K[x]$ such that $f(0) \neq 0$, the image $\psi(f)$ is invertible in $E$, because the power series defining $f^{-1}$ is convergent in $E$. It follows that there is a unique homomorphism $\bar{\psi}: A \Sigma^{-1} \rightarrow E$ extending $\psi$. Here the algebra $A \Sigma^{-1}$ is the universal localization of $A$ with respect to $\Sigma$, that is, the algebra obtained from $A$ by formally inverting all the polynomials in $\Sigma$; see 19 and 35. It turns out that the algebra $A \Sigma^{-1}$ is a von Neumann regular ring, and that the map $\bar{\psi}$ is also injective, thus providing a concrete faithful representation of it. The algebra $A \Sigma^{-1}$ can be identified with the algebra $Q_{K}\left(E_{1}\right)$ of [7, associated with the quiver $E_{1}$ described below (see Figure 1).

An algebra similar to $A \Sigma^{-1}$ was used in 28] to give the first example of a von Neumann regular ring with stable rank 2.

The purpose of this paper is to construct a new class of $K$-algebras, yielding a wide generalization of the above (algebraic) Toeplitz algebras. For each finite poset

Received by the editors February 15, 2008.

2000 Mathematics Subject Classification. Primary 16D70; Secondary 16E50, 06F05, 46L80.

Key words and phrases. von Neumann regular ring, poset, primitive monoid, Toeplitz algebra, Leavitt path algebra.

This research was partially supported by the DGI and European Regional Development Fund, jointly, through Project MTM2005-00934, and by the Comissionat per Universitats i Recerca de la Generalitat de Catalunya.

(C)2009 American Mathematical Society 1505

Reverts to public 


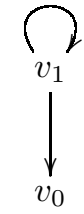

Figure 1 . The quiver $E_{1}$.

$\mathbb{P}$, we will construct a $K$-algebra $Q_{K}(\mathbb{P})$, in such a way that the one corresponding to the poset $\mathbb{P}=\{q, p\}$, where the only nontrivial relation is given by $q<p$, is precisely the algebra $Q_{L}\left(E_{1}\right)$, with $L=K\left(t_{1}, t_{2}, \ldots\right)$ a purely transcendental extension of $K$, and $E_{1}$ is the quiver described before. (For technical reasons, it is convenient to have such an infinite number of variables at our disposal.) In general, there is a natural faithful representation of the algebra $Q_{K}(\mathbb{P})$ on a vector space $V(\mathbb{P})$, which is given locally by Toeplitz operators (Theorem 2.14).

In order to put the construction in a wider perspective we need some preliminary definitions. For any ring $R$, the monoid $\mathcal{V}(R)$ of isomorphism classes of finitely generated projective $R$-modules is always a conical monoid, that is, whenever $x+$ $y=0$, we have $x=y=0$. Recall that an order-unit in a monoid $M$ is an element $u$ in $M$ such that for every $x \in M$ there is a $y \in M$ and $n \geq 1$ such that $x+y=n u$. Observe that, if $R$ is a unital ring, then $[R]$ is a canonical order-unit in $\mathcal{V}(R)$. By results of Bergman [16. Theorems 6.2 and 6.4] and Bergman and Dicks [17, page 315], any conical monoid with an order-unit appears as $\mathcal{V}(R)$ for some unital hereditary ring $R$.

For a von Neumann regular ring $R$, the monoid $\mathcal{V}(R)$ is in addition a refinement monoid. Recall that an abelian monoid $M$ is a refinement monoid in case any equality $x_{1}+x_{2}=y_{1}+y_{2}$ admits a refinement, that is, there are $x_{i j}, 1 \leq i, j \leq 2$ such that $x_{i}=x_{i 1}+x_{i 2}$ and $y_{j}=x_{1 j}+x_{2 j}$ for all $i, j$; see e.g. [13. It is an outstanding open problem to decide whether all countable, conical refinement monoids can be represented as monoids $\mathcal{V}(R)$ for a von Neumann regular ring $R$; see [25, [7]. It was shown by Wehrung in [36] that there are conical refinement monoids of size $\aleph_{2}$ which cannot be represented. We refer the reader to [6] for a recent survey on this problem.

Note that if a monoid $M$ is realizable by a von Neumann regular ring $R$, i.e. $\mathcal{V}(R) \cong M$, and $M$ has an order-unit $u$, then there is an idempotent $e \in M_{n}(R)$, for some $n \geq 1$, which corresponds to $u$ through the isomorphism, and then $e M_{n}(R) e$ is a unital von Neumann regular ring realizing $M$.

A class of monoids whose members are expected to be realizable is the class of finitely generated conical refinement monoids. These monoids enjoy good monoidtheoretic properties, the most important being that they are primely generated [18, Corollary 6.8]. Recall that every monoid $M$ is endowed with a natural pre-order, i.e., the so-called algebraic pre-order, given by $x \leq y$ if and only if there is $z \in M$ such that $y=x+z$. A prime element in an (abelian) monoid $M$ is an element $p$ such that $p \not \leq 0$ and, whenever $p \leq a+b$, then either $p \leq a$ or $p \leq b$. The monoid $M$ is said to be primely generated in case every element of $M$ is a sum of primes. Note that a finitely generated monoid always has an order-unit, namely the sum of a finite generating set. 
We say that $M$ is antisymmetric in case the algebraic pre-order $\leq$ is actually a partial order. Observe that every antisymmetric monoid is conical.

A primitive monoid is a primely generated antisymmetric refinement monoid. A primitive monoid is completely determined by its set of primes $\mathbb{P}(M)$ together with a transitive and antisymmetric relation $\triangleleft$ on it, given by

$$
q \triangleleft p \Longleftrightarrow p+q=p .
$$

Indeed given such a pair $(\mathbb{P}, \triangleleft)$, the abelian monoid $M(\mathbb{P}, \triangleleft)$ defined by taking as a set of generators $\mathbb{P}$ and with relations given by $p=p+q$ whenever $q \triangleleft p$, is a primitive monoid, and the correspondences $M \mapsto(\mathbb{P}(M), \triangleleft)$ and $(\mathbb{P}, \triangleleft) \mapsto M(\mathbb{P}, \triangleleft)$ provide a bijection between isomorphism types of primitive monoids and isomorphism types of pairs $(\mathbb{P}, \triangleleft)$, where $\mathbb{P}$ is a set and $\triangleleft$ a transitive antisymmetric relation on $\mathbb{P}$; see 32, Proposition 3.5.2].

Let $M$ be a primitive monoid and $p \in \mathbb{P}(M)$. Then $p$ is said to be free in case $p \nless p$. Otherwise $p$ is regular; see [14, Section 2]. In case all the primes of $M$ are free, the relation $\triangleleft$ is completely determined by the poset $(\mathbb{P}(M), \leq)$, where $\leq$ is the restriction to $\mathbb{P}(M)$ of the algebraic order of $M$. Namely, we have $q \triangleleft p$ if and only if $q<p$. In this way we obtain mutually inverse (up to isomorphism) correspondences $M \mapsto(\mathbb{P}(M), \leq)$ and $(\mathbb{P}, \leq) \mapsto M(\mathbb{P})$ between primitive monoids having all primes free and posets.

We can now describe the properties of the class of $K$-algebras $Q_{K}(\mathbb{P})$ that we associate with finite posets $\mathbb{P}$. The main result of this paper is:

Theorem. Let $M$ be a finitely generated primitive monoid such that all primes of $M$ are free and let $K$ be a field. Let $\mathbb{P}$ be the finite set of primes of $M$, endowed with the restriction of the algebraic order on $M$. Let $Q_{K}(\mathbb{P})$ be the $K$-algebra described explicitly in Definition 2.1. Then $Q_{K}(\mathbb{P})$ is a von Neumann regular ring and the natural monoid homomorphism

$$
\psi: M \rightarrow \mathcal{V}\left(Q_{K}(\mathbb{P})\right)
$$

is an isomorphism.

This gives a positive solution to the realization problem for the class of finitely generated primitive monoids with all prime elements free. The connection of our work with the paper [7] is as follows. In [7], a von Neumann regular algebra $Q_{K}(E)$ has been attached to every quiver and every field $K$, in such a way that there is an isomorphism $\mathcal{V}\left(Q_{K}(E)\right) \cong M(E)$. Here $M(E)$ is a certain conical refinement monoid associated with the quiver $E$, with generators and relations given explicitly from the combinatorial structure of $E$. It has been shown in 14 that a finitely generated primitive monoid $M$ is isomorphic to a graph monoid $M(E)$ for some quiver $E$ if and only if every free prime of $M$ has at most one free lower cover. (Here a lower cover of a prime $p$ is a prime $q$ such that $q<p$ and $[q, p]=\{q, p\}$.) In particular the primitive monoid $M$ given by $M=\langle p, a, b: p=p+a=p+b\rangle$ cannot be realized by an algebra $Q_{K}(E)$ corresponding to a quiver $E$. The corresponding poset $\mathbb{P}$ is depicted in Figure 2, Observe that the (free) prime $p$ has two (free) lower covers, namely $a$ and $b$.

Consequently our construction significantly enlarges the class of monoids known to be realizable. Moreover, the algebras $Q_{K}(\mathbb{P})$ have a functorial property with respect to certain morphisms between posets (Proposition 2.6). This is analogous to the functoriality property of the regular algebra of a quiver (see [7, page 234]). It 


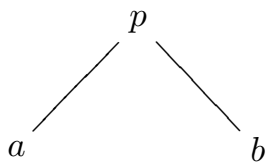

Figure 2. The poset $\mathbb{P}(M)$ for the monoid $M=\langle p, a, b| p=$ $p+a=p+b\rangle$.

is reasonable to expect that a suitable combination of the methods developed in [7] and the methods developed in the present paper will lead to a general construction of a von Neumann regular ring realizing every finitely generated conical refinement monoid. In this direction, it is worth mentioning that the construction of $Q_{K}(\mathbb{P})$ in Definition 2.1 gives an algebra $Q_{L}(E)$ associated with a suitable quiver $E$, where $L=K\left(t_{1}, t_{2}, \ldots,\right)$, in case every element in $\mathbb{P}$ has at most one lower cover. In particular, for the poset with two elements $q, p$ with $q<p$, we get the Toeplitz algebra $Q_{L}\left(E_{1}\right)$ mentioned at the beginning of the introduction. Certainly the next step in the realization problem consists of realizing all the finitely generated primitive monoids. A monoid $M$ in this class, which is not covered by the results in [7] or the results in the present paper, must have both regular and free primes, and some free prime of $M$ must have at least two free lower covers. For instance, the monoid

$$
M=\langle q, p, a, b \mid q=2 q=q+p, p=p+a=p+b\rangle
$$

satisfies these properties.

Although the main construction in the present paper shares some resemblances with the one in 7 - both objects have the form $\mathcal{A} \Upsilon^{-1}$, where $\mathcal{A}$ is the path algebra of a quiver in [7, and $\mathcal{A}$ is an algebra described by generators and relations coming from the structure of a poset in the present paper (see Definition 2.1), and $\Upsilon$ is a certain set of morphisms between finitely generated projective $\mathcal{A}$-modules - a completely new set of techniques has been developed to deal with our construction here. These techniques include the fundamental study (in Section 3) of the conditions for the preservation of pullbacks under the functor $\mathcal{V}(-)$, which indeed has dictated the form of the relations used in Definition 2.1 to define the algebra $\mathcal{A}$.

We now summarize the contents of the paper. In Section 11 we review some basic definitions and results on posets, monoids and rings. Section 2 contains the definition of the $K$-algebra $Q_{K}(\mathbb{P})$ associated to a finite poset $\mathbb{P}$ and analyzes the functorial behaviour and algebraic properties of this construction. Moreover, we give a Toeplitz-like representation of this algebra.

Sections 3 and 4 contain the technical results needed to gain control on the relationship between von Neumann regular rings $R$ and their monoids $\mathcal{V}(R)$ under natural categorical operations such as pullbacks and pushouts. These results are of independent interest and most likely will play a role in future developments of the theory.

Section [5]develops a generalization of the construction in [7] for a particular class of quivers, which will be used in the proof of our main result. This generalized construction can be studied in a more general setting, see [8], but we present here a direct approach to the results which are needed in the present paper. 
Finally, Section 6 contains the proof of our main result, which is based upon a reconstruction technique of a finite poset from the family of its maximal chains. The technical tools developed in the previous sections enable us to mimic the mentioned reconstruction at the ring level and at the monoid level.

\section{Preliminary definitions}

All rings in this paper will be associative and all monoids will be abelian. A (not necessarily unital) ring $R$ is von Neumann regular if for every $a \in R$ there is $b \in R$ such that $a=a b a$. Our basic reference for the theory of von Neumann regular rings is 24 .

For a (not necessarily unital) ring $R$, let $M_{\infty}(R)$ be the directed union of $M_{n}(R)$ $(n \in \mathbb{N})$, where the transition maps $M_{n}(R) \rightarrow M_{n+1}(R)$ are given by $x \mapsto\left(\begin{array}{cc}x & 0 \\ 0 & 0\end{array}\right)$. Two idempotents $e, f \in M_{\infty}(R)$ are equivalent in case there are $x \in e M_{\infty}(R) f$ and $y \in f M_{\infty}(R) e$ such that $x y=e$ and $y x=f$. We define $\mathcal{V}(R)$ to be the set of equivalence classes $\mathcal{V}(e)$ of idempotents $e$ in $M_{\infty}(R)$ with the operation

$$
\mathcal{V}(e)+\mathcal{V}(f):=\mathcal{V}\left(\left(\begin{array}{ll}
e & 0 \\
0 & f
\end{array}\right)\right)
$$

for idempotents $e, f \in M_{\infty}(R)$. The classes $\mathcal{V}(e)$ are often denoted also by $[e]$. For a unital $R$, the monoid $\mathcal{V}(R)$ is the monoid of isomorphism classes of finitely generated projective left $R$-modules, where the operation is induced by direct sum. If $I$ is an ideal of a unital ring $R$, then $\mathcal{V}(I)$ can be identified with the monoid of isomorphism classes of finitely generated projective left $R$-modules $P$ such that $P=I P$.

If $R$ is an exchange ring (in particular, if $R$ is von Neumann regular), then $\mathcal{V}(R)$ is a conical refinement monoid; see [11, Corollary 1.3]. (This is true even in the nonunital case by [5, Proposition 1.5(b)].)

Let $M$ be a monoid. An order-ideal of $M$ is a nonempty subset $I$ of $M$ such that $x+y \in I$ iff $x \in I$ and $y \in I$, for all $x, y \in M$. In this case, the equivalence relation $\equiv_{I}$ defined on $M$ by the rule

$$
x \equiv_{I} y \Longleftrightarrow(\exists u, v \in I)(x+u=y+v), \quad \text { for all } x, y \in M
$$

is a monoid congruence of $M$. We put $M / I=M / \equiv_{I}$ and we shall say that $M / I$ is an ideal quotient of $M$. We denote by

$$
M \mid a=\left\{x \in M:\left(\exists n \in \mathbb{Z}^{+}\right)(x \leq n a)\right\}
$$

the order-ideal generated by an element $a \in M$. Similarly $M \mid S$ will denote the order-ideal of $M$ generated by a subset $S$ of $M$.

When $M$ is a conical refinement monoid, the set $\mathcal{L}(M)$ of order-ideals of $M$ forms a complete distributive lattice, with suprema and infima given by the sum and the intersection of order-ideals, respectively.

Let us denote by $\mathcal{L}(R)$ the lattice of (two-sided) ideals of $R$, and by $\mathcal{L}(M)$ the lattice of order-ideals of $M$.

Proposition 1.1 (cf. [11, Proposition 1.4]). If $R$ is von Neumann regular, then there is a lattice isomorphism $\mathcal{L}(R) \rightarrow \mathcal{L}(\mathcal{V}(R)), I \mapsto \mathcal{V}(I)$ from $\mathcal{L}(R)$ onto $\mathcal{L}(\mathcal{V}(R))$. Moreover $\mathcal{V}(R / I) \cong \mathcal{V}(R) / \mathcal{V}(I)$ for any ideal $I$ of $R$.

Say that a subset $A$ of a poset $\mathbb{P}$ is a lower subset in case $q \leq p$ and $p \in A$ imply $q \in A$. Again the set $\mathcal{L}(\mathbb{P})$ of all lower subsets of $\mathbb{P}$ forms a complete distributive lattice, which is a sublattice of the Boolean lattice $\mathbf{2}^{\mathbb{P}}$. 
If $M$ is a primitive monoid, then the set of primes of $M, \mathbb{P}(M)$, is a poset with the partial order $\leq$ induced from the algebraic order of $M$, and we easily get:

Proposition 1.2. For a primitive monoid $M$, there is a lattice isomorphism

$$
\mathcal{L}(M) \cong \mathcal{L}(\mathbb{P}(M)), \quad S \mapsto \mathbb{P}(S)=\mathbb{P}(M) \cap S .
$$

For an element $p$ of a poset $\mathbb{P}$, write

$$
\mathrm{L}(p)=\mathrm{L}(\mathbb{P}, p)=\{q \in \mathbb{P}: q<p \text { and }[q, p]=\{q, p\}\} .
$$

An element of $\mathrm{L}(p)$ is called a lower cover of $p$.

For any prime element $p$ in a refinement monoid $M$, the map

$$
\phi_{p}: M \rightarrow \mathbb{Z}^{\infty}, \quad x \mapsto \sup \left(n \in \mathbb{Z}^{+} \mid n p \leq x\right)
$$

is a monoid homomorphism from $M$ to $\mathbb{Z}^{\infty}:=\mathbb{Z}^{+} \cup\{\infty\}$; see [18, Theorem 5.4]. Furthermore, if $M$ is primitive, then the map

$$
\phi: M \rightarrow\left(\mathbb{Z}^{\infty}\right)^{\mathbb{P}(M)}, \quad x \mapsto\left(\phi_{p}(x) \mid p \in \mathbb{P}(M)\right)
$$

is a monoid embedding as well as an $\triangleleft$-embedding; see [18, Theorem 5.11] or [37, Corollary 6.14].

A monoid $M$ is said to be separative in case, whenever $a, b \in M$ and $a+a=$ $a+b=b+b$, then we have $a=b$. Similarly $M$ is strongly separative in case $a+a=a+b$ implies $a=b$ for $a, b \in M$. A ring $R$ is said to be (strongly) separative in case $\mathcal{V}(R)$ is (strongly) separative; see [11] for background and various equivalent conditions. Every primely generated refinement monoid is separative [18, Theorem 4.5]. In particular every primitive monoid is separative. Moreover, a primitive monoid $M$ is strongly separative if and only if all the primes in $M$ are free; see [18, Theorem 4.5, Corollary 5.9]. Thus, the class of monoids that we will realize in this paper (as monoids of projectives over regular rings) coincides exactly with the strongly separative primitive monoids.

\section{The algebras $Q_{K}(\mathbb{P})$}

Recall from the introduction that (finitely generated) primitive monoids $M$ with all primes free are determined by the (finite) posets $\mathbb{P}(M)$ of their prime elements. The construction below has functorial properties with respect to some maps of posets, so it is better thought of as a functor from finite posets to $K$-algebras. However we will use both notations $Q_{K}(M)$ and $Q_{K}(\mathbb{P}(M))$ interchangeably.

Definition 2.1. Let $M$ be a finitely generated primitive monoid with all primes free, and let $\mathbb{P}$ be its finite poset of primes. Fix a field $K$. For $p \in \mathbb{P}$ denote $n_{p}:=|\mathrm{L}(\mathbb{P}, p)|$.

Let $L=K\left(t_{1}, t_{2}, \ldots,\right)$ be an infinite purely transcendental extension of $K$. Let $\mathcal{A}_{0}$ be the semisimple $L$-algebra generated by a family of orthogonal idempotents $\{e(p): p \in \mathbb{P}\}$ with sum 1 , and, for each $p \in \mathbb{P}$ with $n_{p}>0$, a family of orthogonal idempotents $\{e(p, q): q \in \mathrm{L}(p)\}$ such that $e(p) e(p, q)=e(p, q)=e(p, q) e(p)$.

For convenience, put $e^{\prime}(p):=e(p)-\sum_{q \in \mathrm{L}(p)} e(p, q)$, with $e^{\prime}(p)=e(p)$ in case $n_{p}=0$. Then we have

$$
\mathcal{A}_{0}:=\prod_{p \in \mathbb{P}} e^{\prime}(p) L \times \prod_{p \in \mathbb{P}} \prod_{q \in \mathrm{L}(p)} e(p, q) L,
$$


and we also have an orthogonal decomposition

$$
e(p)=e^{\prime}(p)+\sum_{q \in \mathrm{L}(p)} e(p, q) \quad(p \in \mathbb{P}) .
$$

Let $\mathcal{A}_{1}$ be the $L$-algebra generated by $\mathcal{A}_{0}$ and a family $\left\{\alpha_{p, q}: p \in \mathbb{P}, q \in \mathrm{L}(p)\right\}$, subject to the following relations:

$$
\begin{gathered}
\alpha_{p, q} e(p)=\alpha_{p, q}=(e(p)-e(p, q)) \alpha_{p, q} \quad(p \in \mathbb{P}, q \in \mathrm{L}(p)), \\
\alpha_{p, q^{\prime}} e(p, q)=e(p, q) \alpha_{p, q^{\prime}} \quad \text { for } q \neq q^{\prime} \\
\alpha_{p, q^{\prime}} \alpha_{p, q}=\alpha_{p, q} \alpha_{p, q^{\prime}} \quad\left(q, q^{\prime} \in \mathrm{L}(p)\right)
\end{gathered}
$$

For each polynomial $f\left(x_{q}\right) \in L\left[x_{q}: q \in \mathrm{L}(p)\right]$ in commuting variables $\left\{x_{q}\right.$ : $q \in \mathrm{L}(p)\}$ and each $q^{\prime} \in \mathrm{L}(p)$, write $v_{q^{\prime}}(f)$ for the valuation of $f\left(x_{q}\right)$, seen as a polynomial in the one-variable polynomial ring $\left(L\left[x_{q}: q \neq q^{\prime}\right]\right)\left[x_{q^{\prime}}\right]$, at the ideal generated by $x_{q^{\prime}}$. In other words, $v_{q^{\prime}}(f)$ is the highest integer $n$ such that $x_{q^{\prime}}^{n}$ divides $f$. Write

$$
v(f)=\max \left\{v_{q^{\prime}}(f): q^{\prime} \in \mathrm{L}(p)\right\} .
$$

Let $\Sigma(p)$ be the set of all elements of $e(p) \mathcal{A}_{1} e(p)$ given by

$$
\Sigma(p)=\left\{f\left(\alpha_{p, q}\right): v(f)=0\right\} .
$$

Set

$$
\Sigma:=\bigcup_{p \in \mathbb{P}} \Sigma(p) .
$$

Let $\mathcal{T}(\mathbb{P})$ be the quiver having as vertices the elements of $\mathbb{P}$ and having one arrow from $p$ to $q$ if and only if $q \in \mathrm{L}(p)$. We assume our posets are endowed with bijective $\operatorname{maps}\left\{1, \ldots, n_{p}\right\} \rightarrow s_{\mathcal{T}(\mathbb{P})}^{-1}(p)$ for every $p \in \mathbb{P}$ such that $n_{p}>0$. For convenience, we will refer to the enhanced structure as a labelled poset.

For $p \in \mathbb{P}$ with $n_{p}>0$, we denote by $\left(p, q_{i}\right)$ the image of $i \in\left\{1, \ldots, n_{p}\right\}$ under the given map $\left\{1, \ldots, n_{p}\right\} \rightarrow s_{\mathcal{T}(\mathbb{P})}^{-1}(p)$; we also denote by $\sigma^{p}: L \rightarrow L$ the $K$-algebra endomorphism determined by the rule $\sigma^{p}\left(t_{i}\right)=t_{i+n_{p}-1}$, and by

$$
\sigma_{j}:\left\{1, \ldots, n_{p}\right\} \longrightarrow\left\{1, \ldots, n_{p}-1\right\}
$$

the surjective nondecreasing map sending $j$ and $j+1$ to $j$, for $j<n_{p}$, and with $\sigma_{n_{p}}:=\sigma_{n_{p}-1}$.

We consider the $K$-algebra $\mathcal{A}$ obtained by adjoining to $\mathcal{A}_{1}$ a new family of generators $\left\{\beta_{p, q}: p \in \mathbb{P}, q \in \mathrm{L}(p)\right\}$, subject to the relations:

$$
\begin{gathered}
\lambda \beta_{p, q}=\beta_{p, q} \sigma^{p}(\lambda) \quad(\lambda \in L, q \in \mathrm{L}(p)), \\
\alpha_{p, q_{\ell}} \beta_{p, q_{j}}=\beta_{p, q_{j}} t_{\sigma_{j}(\ell)}, \quad\left(j, \ell \in\left\{1, \ldots, n_{p}\right\}, j \neq \ell\right)
\end{gathered}
$$

and

$$
e(p, q) \beta_{p, q}=\beta_{p, q}=\beta_{p, q} e(q) \quad(q \in \mathrm{L}(p)) .
$$

Next consider the set of homomorphisms of finitely generated projective left $\mathcal{A}$ modules $\Sigma_{1}=\left\{\mu_{p, q}: p \in \mathbb{P}, q \in \mathrm{L}(p)\right\}$, where for $q \in \mathrm{L}(p)$, the map $\mu_{p, q}$ is defined as follows:

$$
\mu_{p, q}: \mathcal{A} e(p) \longrightarrow \mathcal{A} e(p) \oplus \mathcal{A} e(q), \quad \mu_{p, q}(r)=\left(r \alpha_{p, q}, r \beta_{p, q}\right) .
$$


Finally define the $K$-algebra $Q_{K}(\mathbb{P})=Q_{K}(M)$ associated with $\mathbb{P}$ (or equivalently, with $M$ ) as

$$
Q_{K}(\mathbb{P})=Q_{K}(M)=\mathcal{A}\left(\Sigma \cup \Sigma_{1}\right)^{-1},
$$

where the elements in $\Sigma(p)$ are seen as left $\mathcal{A}$-module morphisms $\mathcal{A} e(p) \rightarrow \mathcal{A} e(p)$. The algebra $Q_{K}(\mathbb{P})$ is a unital $K$-algebra, with unit $1=\sum_{p \in \mathbb{P}} e(p)$.

Let us write explicitly what is the meaning of making the maps in $\Sigma_{1}$ invertible in terms of generators and relations. It amounts to adding to $\mathcal{A} \Sigma^{-1}$ a family of generators $\bar{\beta}_{p, q}, \bar{\alpha}_{p, q}$ for $q \in \mathrm{L}(p), p \in \mathbb{P}$ with the following relations:

$$
e(p) \bar{\alpha}_{p, q}=\bar{\alpha}_{p, q}=\bar{\alpha}_{p, q}(e(p)-e(p, q)), \bar{\alpha}_{p, q} \alpha_{p, q}=e(p), \alpha_{p, q} \bar{\alpha}_{p, q}=e(p)-e(p, q),
$$

$$
e(q) \bar{\beta}_{p, q}=\bar{\beta}_{p, q}=\bar{\beta}_{p, q} e(p, q), \quad \bar{\beta}_{p, q} \beta_{p, q}=e(q), \quad \beta_{p, q} \bar{\beta}_{p, q}=e(p, q) .
$$

It is a simple matter to check that the relations above imply the following ones:

$$
\begin{aligned}
& e(p, q) \bar{\alpha}_{p, q^{\prime}}=\bar{\alpha}_{p, q^{\prime}} e(p, q) \quad \text { for } q \neq q^{\prime}, \quad \bar{\alpha}_{p, q^{\prime}} \bar{\alpha}_{p, q}=\bar{\alpha}_{p, q} \bar{\alpha}_{p, q^{\prime}} \quad\left(q, q^{\prime} \in \mathrm{L}(p)\right) \text {, } \\
& \bar{\beta}_{p, q} \lambda=\sigma^{p}(\lambda) \bar{\beta}_{p, q} \quad(\lambda \in L, q \in \mathrm{L}(p)), \\
& \bar{\beta}_{p, q_{j}} \alpha_{p, q_{\ell}}=t_{\sigma_{j}(\ell)} \bar{\beta}_{p, q_{j}}, \quad\left(j, \ell \in\left\{1, \ldots, n_{p}\right\}, j \neq \ell\right), \\
& t_{\sigma_{j}(\ell)}^{-1} \bar{\beta}_{p, q_{j}}=\bar{\beta}_{p, q_{j}} \bar{\alpha}_{p, q_{\ell}}, \quad\left(j, \ell \in\left\{1, \ldots, n_{p}\right\}, j \neq \ell\right) .
\end{aligned}
$$

Remark 2.2. Observe that the above relations (2.13), (2.14), (2.15), (2.16), (2.18) give that the $K$-algebra $\mathcal{A} \Sigma_{1}^{-1}$ admits a natural involution $x \mapsto \bar{x}$ which is the identity on $\prod_{p \in \mathbb{P}} e^{\prime}(p) K \times \prod_{p \in \mathbb{P}} \prod_{q \in \mathrm{L}(p)} e(p, q) K$, and sends $t_{k}$ to $t_{k}^{-1}, \alpha_{p, q}$ to $\bar{\alpha}_{p, q}$, and $\beta_{p, q}$ to $\bar{\beta}_{p, q}$. The algebra $\mathcal{A} \Sigma_{1}^{-1}$ is analogous to the Leavitt path algebra of [2] (see also [13]).

Since $Q_{K}(M) e(p) \cong Q_{K}(M) e(p) \oplus Q_{K}(M) e(q)$ for every $q \in \mathrm{L}(p)$, there is a unique monoid homomorphism $\psi: M \rightarrow \mathcal{V}\left(Q_{K}(M)\right)$ such that $\psi(p)=[e(p)]$. Our main result can now be stated as follows:

Theorem 2.3. Let $M$ be a finitely generated primitive monoid such that all primes of $M$ are free and let $K$ be a field. Then $Q_{K}(M)=Q_{K}(\mathbb{P}(M))$ is a von Neumann regular ring and the natural monoid homomorphism

$$
\psi: M \rightarrow \mathcal{V}\left(Q_{K}(M)\right)
$$

is an isomorphism.

This will be proven in Section 6 .

Remark 2.4. (1) We have considered the structure of a labelled poset in order to define relation (2.9), so that strictly speaking we have defined an algebra $Q_{K}(\mathbb{P})$ for each labelled poset $\mathbb{P}$. In order to consider the assignment $\mathbb{P} \mapsto$ $Q_{K}(\mathbb{P})$ as a functor, this ingredient is needed. However the properties of $Q_{K}(\mathbb{P})$ do not depend on the chosen maps.

(2) In case $\mathbb{P}$ is a forest, i.e. $\mathbb{P} \downarrow p:=\{q \in \mathbb{P} \mid q \leq p\}$ is a chain for any $p \in \mathbb{P}$, we get that $Q_{K}(\mathbb{P})$ coincides with $Q_{L}(E)$, the regular algebra of $E$ over the field $L$, see Section 5 and [7, where $E$ is the quiver obtained by adding to $\mathcal{T}(\mathbb{P})$ a loop at each vertex $p \in \mathbb{P}$ such that $n_{p}>0$. For instance the quiver $E$ corresponding to the forest $\mathbb{P}=\{q, p\}$, where $q<p$, is the quiver $E_{1}$ of 
Figure 1. The Leavitt path algebra of $E$ over the field $L$, see [2], is precisely the algebra $\mathcal{A} \Sigma_{1}^{-1}$ in the notation of Definition 2.1.

Example 2.5. We consider our motivating example $M=\langle p, a, b \mid p=p+a=p+b\rangle$; see Figure 2, Observe that $\mathbb{P}:=\mathbb{P}(M)$ is the poset $\{p, a, b\}$ with $a<p$ and $b<p$, so that $n_{p}=2$ and $n_{a}=n_{b}=0$, so that $\mathbb{P}$ is not a forest, and the monoid $M$ is not a graph monoid ([14, Theorem 5.1]). We choose the map $\{1,2\} \rightarrow \mathrm{L}(p)=\{a, b\}$ given by $1 \mapsto a$ and $2 \mapsto b$. The regular algebra $Q_{K}(\mathbb{P})$ associated with the poset $\mathbb{P}$ can be described in terms of the regular algebras $Q_{1}:=Q_{K}\left(S_{1}\right)$ and $Q_{2}:=Q_{K}\left(S_{2}\right)$ associated with the forests $S_{1}$ and $S_{2}$, where $S_{1}$ and $S_{2}$ are the chains $\{a, p\}$ and $\{b, p\}$, respectively. Since this is the main point of the whole construction, we are going to provide some hints on the general strategy for proving Theorem 2.3 in this particularly simple case.

Note that, by Remark 2.4 (2), the algebras $Q_{1}$ and $Q_{2}$ are isomorphic to the regular $L$-algebra $Q_{L}\left(E_{1}\right)$ attached to the quiver $E_{1}$ of Figure 1 . Note that $Q_{1}$ has unit element $1_{Q_{1}}=e(p)+e(a)$, and has generators $\alpha_{1}, \bar{\alpha}_{1}, \beta_{1}, \bar{\beta}_{1}$, with

$$
\bar{\alpha}_{1} \alpha_{1}=e(p)=\alpha_{1} \bar{\alpha}_{1}+\beta_{1} \bar{\beta}_{1}
$$

and $\bar{\beta}_{1} \beta_{1}=e(a)$. Moreover, all elements of the form $e(p) f\left(\alpha_{1}\right)$, where $f \in L[z]$ is a polynomial with nonzero constant term, are in $\Sigma(p)$ and thus the elements $e(p) f\left(\alpha_{1}\right)$ are invertible in $e(p) Q_{1} e(p)$. Let $I_{1}$ be the ideal of $Q_{1}$ generated by $e(a)$. It follows from the above that $Q_{1} / I_{1} \cong L(z)$, the rational function field in one variable $z$, where $z$ corresponds to the class of $\alpha_{1}$ in $Q_{1} / I_{1}$. Since $L(z) \cong L$, we may consider a surjective homomorphism $\pi_{1}: Q_{1} \rightarrow L$ such that $\pi_{1}\left(\alpha_{1}\right)=t_{1}$, and $\pi_{1}\left(t_{i}\right)=t_{i+1}$ for all $i$. Note that the kernel of $\pi_{1}$ is $I_{1}$. Similarly, we have a surjective homomorphism $\pi_{2}: Q_{2} \rightarrow L$, with kernel $I_{2}:=Q_{2} e(b) Q_{2}$ sending $\alpha_{2}$ to $t_{2}, t_{1}$ to $t_{1}$ and $t_{i}$ to $t_{i+1}$ for all $i>1$, where $\alpha_{2}, \bar{\alpha}_{2}, \beta_{2}, \bar{\beta}_{2}$ are canonical generators for $Q_{2}$. It is a simple exercise to show that there is a pullback diagram:

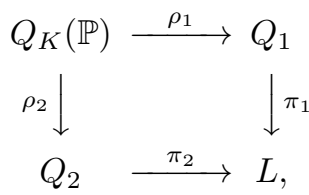

for suitably defined $K$-algebra homomorphisms $\rho_{i}: Q_{K}(\mathbb{P}) \rightarrow Q_{i}$. (The key here is to define $\rho_{1}\left(\alpha_{p, b}\right)=e(p) t_{1}$, and $\rho_{1}\left(e(p) t_{i}\right)=e(p) t_{i+1}, \rho_{1}\left(e(a) t_{i}\right)=e(a) t_{i}$ for all $i$, and similarly with $\rho_{2}$, so that the defining relations of $Q_{K}(\mathbb{P})$ are preserved by $\rho_{i}$, and $\pi_{1} \circ \rho_{1}=\pi_{2} \circ \rho_{2}$.)

The results in Section 3 guarantee that the functor $\mathcal{V}$ sends the pullback diagram (2.19) to a pullback diagram of monoids (see Example 3.3), and then the results in [7] applied to the quiver algebras $Q_{i}$ enable us to conclude that $\mathcal{V}\left(Q_{K}(\mathbb{P})\right) \cong M$, as desired.

The universal property of the construction is as follows. Call a morphism of posets $f: \mathbb{P} \rightarrow \mathbb{P}^{\prime}$ a complete homomorphism in case it is injective and has the property that for any $p \in \mathbb{P}$ such that $\mathrm{L}(\mathbb{P}, p) \neq \emptyset$ we have that $f$ restricts to a bijection from $\mathrm{L}(\mathbb{P}, p)$ onto $\mathrm{L}\left(\mathbb{P}^{\prime}, f(p)\right)$. Observe that this is the same as a complete graph homomorphism $\mathcal{T}(\mathbb{P}) \rightarrow \mathcal{T}\left(\mathbb{P}^{\prime}\right)$ between the quivers introduced in Definition 2.1, in the sense of [13, Section 3]. If $\mathbb{P}$ and $\mathbb{P}^{\prime}$ are labelled posets, we define a complete homomorphism as a complete graph homomorphism $f: \mathcal{T}(\mathbb{P}) \rightarrow \mathcal{T}\left(\mathbb{P}^{\prime}\right)$ respecting the labels. 
The category of nonunital $K$-algebras has as objects all not necessarily unital $K$-algebras, and as morphisms all the homomorphisms of $K$-algebras. In the next proposition, note that the algebras $Q_{K}(\mathbb{P})$ are unital, but the homomorphisms appearing there are not unital except in the trivial cases.

Proposition 2.6. The map $\mathbb{P} \mapsto Q_{K}(\mathbb{P})$ extends to a functor from the category of finite labelled posets and complete homomorphisms to the category of nonunital $K$-algebras.

Proof. Straightforward. Observe that, for a complete homomorphism $f: \mathbb{P} \rightarrow \mathbb{P}^{\prime}$, the image of $1_{Q_{K}(\mathbb{P})}$ is the idempotent $\sum_{p \in \mathbb{P}} e(f(p))$.

Remark 2.7. Using the functoriality established in Proposition 2.6. one can generalize Theorem 2.3 to some infinite posets by taking direct limits; see for instance the proofs of [13, Theorem 3.5] and [7, Theorem 4.4]. Note that the class of (labelled) posets that can be obtained as a direct limit of a directed system of finite labelled posets, with complete homomorphisms as transition maps, includes the class of lower finite posets, where a poset $\mathbb{P}$ is lower finite in case the subset $\mathbb{P} \downarrow p=\{q \in \mathbb{P} \mid q \leq p\}$ is finite, for any $p \in P$. A characterization of graph monoids among primitive monoids $M$ such that $\mathbb{P}(M)$ is lower finite has been obtained in [14, Theorem 5.1].

We start analyzing the basic algebraic properties of $Q_{K}(\mathbb{P})$. We begin with a general observation, stating that universal localization commutes with adjoining generators and relations to a given algebra. For a field $K$, the coproduct of two unital $K$-algebras $\mathcal{A}$ and $\mathcal{B}$ will be denoted by $\mathcal{A} *_{K} \mathcal{B}$; see [15] for the definition and properties of such coproducts.

Proposition 2.8. Let $\mathcal{A}$ be a unital $K$-algebra over a field $K$, and let $\Sigma$ be a set of morphisms between finitely generated projective $\mathcal{A}$-modules. Let $\mathcal{B}$ be another unital $K$-algebra, and let $I$ be an ideal of the coproduct $\mathcal{A} *_{K} \mathcal{B}$. Set $\mathcal{C}=\left(\mathcal{A} *_{K} \mathcal{B}\right) / I$. Let $\bar{I}$ be the ideal of $\left(\mathcal{A} \Sigma^{-1}\right) *_{K} \mathcal{B}$ generated by the image of I under the canonical map $\mathcal{A} *_{K} \mathcal{B} \rightarrow\left(\mathcal{A} \Sigma^{-1}\right) *_{K} \mathcal{B}$, and let $\bar{\Sigma}$ be the image of $\Sigma$ under the natural map $\mathcal{A} \rightarrow \mathcal{C}$ (obtained by composing the natural map $\mathcal{A} \rightarrow \mathcal{A} *_{K} \mathcal{B}$ with the canonical projection $\left.\mathcal{A} *_{K} \mathcal{B} \rightarrow \mathcal{C}\right)$. It follows that there is a natural isomorphism $\mathcal{C} \bar{\Sigma}^{-1} \cong\left(\mathcal{A} \Sigma^{-1} *_{K} \mathcal{B}\right) / \bar{I}$.

Proof. First we show that $\mathcal{A} \Sigma^{-1} *_{K} \mathcal{B} \cong\left(\mathcal{A} *_{K} \mathcal{B}\right) \widetilde{\Sigma}^{-1}$ (over $\left.\mathcal{A} *_{K} \mathcal{B}\right)$, where $\widetilde{\Sigma}$ is the image of $\Sigma$ under the map $\mathcal{A} \rightarrow \mathcal{A} *_{K} \mathcal{B}$. This follows from the easily proved fact that both algebras satisfy an obvious universal condition with respect to $K$-algebra homomorphisms $\psi_{1}: \mathcal{A} \rightarrow S$ and $\psi_{2}: \mathcal{B} \rightarrow S$, with $\psi_{1}$ being $\Sigma$-inverting.

Let $\widetilde{I}$ be the ideal of $\left(\mathcal{A} *_{K} \mathcal{B}\right) \widetilde{\Sigma}^{-1}$ generated by the image of $I$. By 21, Proposition 4.3], we have $\left(\left(\mathcal{A} *_{K} \mathcal{B}\right) \widetilde{\Sigma}^{-1}\right) / \widetilde{I} \cong \mathcal{C} \bar{\Sigma}^{-1}$ and thus combining with the above isomorphism we get

$$
\mathcal{C} \bar{\Sigma}^{-1} \cong\left(\left(\mathcal{A} *_{K} \mathcal{B}\right) \widetilde{\Sigma}^{-1}\right) / \widetilde{I} \cong\left(\mathcal{A} \Sigma^{-1} *_{K} \mathcal{B}\right) / \bar{I},
$$

as desired.

Lemma 2.9. For $p \in \mathbb{P}, q \in \mathrm{L}(p)$ and $f \in \Sigma(p)$ we have

$$
e(p, q) f^{-1}=\left(f_{0}^{\prime}\right)^{-1} \bar{w} e(p, q)=e(p, q)\left(f_{0}^{\prime}\right)^{-1} \bar{w}
$$

and

$$
\bar{\alpha}_{p, q} f^{-1}=f^{-1} \bar{\alpha}_{p, q}+f^{-1}\left(f_{0}^{\prime}\right)^{-1} g \bar{w} e(p, q)
$$


where $w$ is a monomial in $\left\{\alpha_{p, q^{\prime}} \mid q^{\prime} \neq q\right\}$, and $f_{0}^{\prime} \in L\left[\alpha_{p, q^{\prime}}: q^{\prime} \neq q\right] \cap \Sigma(p)$, and $g \in L\left[\alpha_{p, q^{\prime}}\right]$.

Proof. Write

$$
f=f_{0}+\alpha_{p, q} f_{1}+\cdots+\alpha_{p, q}^{m} f_{m},
$$

where $f_{s} \in L\left[\alpha_{p q^{\prime}}: q^{\prime} \neq q\right]$. Then $e(p, q) f=f_{0} e(p, q)$ by relations (2.3) and (2.4). Now write

$$
f_{0}=w f_{0}^{\prime}
$$

with $w$ a monomial in $\left\{\alpha_{p, q^{\prime}}: q^{\prime} \neq q\right\}$ and $f_{0}^{\prime} \in \Sigma(p) \cap L\left[\alpha_{p, q^{\prime}}: q^{\prime} \neq q\right]$. Then we get that $f_{0}$ is left invertible in $e(p) Q_{K}(\mathbb{P}) e(p)$, because $\left(f_{0}^{\prime}\right)^{-1} \bar{w} f_{0}=e(p)$. Thus

$$
e(p, q) f^{-1}=\left(f_{0}^{\prime}\right)^{-1} \bar{w} e(p, q),
$$

as desired. Also $\left(f_{0}^{\prime}\right)^{-1} \bar{w} e(p, q)=e(p, q)\left(f_{0}^{\prime}\right)^{-1} \bar{w}$ because of (2.4) and (2.15).

Now, observe that, since $\bar{\alpha}_{p, q} f_{i}=f_{i} \bar{\alpha}_{p, q}$ for all $i$, we have

$$
\bar{\alpha}_{p, q} f-f \bar{\alpha}_{p, q}=\left(f_{1}+\alpha_{p, q} f_{2}+\cdots+\alpha_{p, q}^{m-1} f_{m}\right) e(p, q)
$$

so that, multiplying this relation on the left and on the right by $f^{-1}$ and using (2.20), we get equation (2.21) with $g=-f_{1}-\alpha_{p, q} f_{2}-\cdots-\alpha_{p, q}^{m-1} f_{m}$.

Observe that Proposition 2.8 says that $Q_{K}(\mathbb{P})$ can be obtained as follows. First consider the commutative $L$-algebra

$$
\mathcal{A}_{2}=\prod_{p \in \mathbb{P}} e(p) L\left[\alpha_{p, q}: q \in \mathrm{L}(p)\right] \Sigma(p)^{-1} .
$$

Then we adjoin to $\mathcal{A}_{2}$ the family of orthogonal idempotents $\{e(p, q): q \in \mathrm{L}(p), p \in$ $\mathbb{P}\}$, with $e(p, q) e(p)=e(p, q)=e(p) e(p, q)$, and elements $\left\{\bar{\alpha}_{p, q}: p \in \mathbb{P}, q \in \mathrm{L}(p)\right\}$, and impose relations (2.3), (2.4) and (2.13), to obtain a new algebra $\mathcal{A}_{3}$. Note that

$$
\mathcal{A}_{3}=\prod_{p \in \mathbb{P}} e(p) \mathcal{A}_{3} e(p) .
$$

Finally the algebra $Q_{K}(\mathbb{P})$ is obtained by adjoining to $\mathcal{A}_{3}$ the generators $\beta_{p, q}, \bar{\beta}_{p, q}$ for $q \in \mathrm{L}(p)$, subject to the relations (2.8), (2.9), (2.10) and (2.14).

Lemma 2.10. With the above notation we have

(1) $e(p) \mathcal{A}_{3} \beta_{p, q} \subseteq \sum_{f \in \Sigma(p)} \sum_{i=0}^{\infty} f^{-1} \alpha_{p, q}^{i} \beta_{p, q} L$.

(2) $\bar{\beta}_{p, q} \mathcal{A}_{3} e(p) \subseteq \sum_{i=0}^{\infty} L \bar{\beta}_{p, q} \bar{\alpha}_{p, q}^{i}$.

(3) $\bar{\beta}_{p, q} \mathcal{A}_{3} \beta_{p, q} \subseteq \operatorname{Le}(q)$.

(4) $\bar{\beta}_{p, q} \mathcal{A}_{3} \beta_{p, q^{\prime}}=0$ for $q \neq q^{\prime}$.

Proof. (1) Write $T_{(p, q)}:=\sum_{f \in \Sigma(p)} \sum_{i=0}^{\infty} f^{-1} \alpha_{p, q}^{i} \beta_{p, q} L$. It suffices to check that $\alpha_{p, q^{\prime}} T_{(p, q)} \subseteq T_{(p, q)}$ and $e\left(p, q^{\prime}\right) T_{(p, q)} \subseteq T_{(p, q)}$ and $\bar{\alpha}_{p, q^{\prime}} T_{(p, q)} \subseteq T_{(p, q)}$. The first relation follows from (2.9). The containment

$$
e\left(p, q^{\prime}\right)\left(\sum_{i=0}^{\infty} \alpha_{p, q}^{i} \beta_{p, q} L\right) \subseteq \sum_{i=0}^{\infty} \alpha_{p, q}^{i} \beta_{p, q} L
$$

follows from (2.3), (2.4) and (2.10). It follows from this containment and from (2.20) that, to show that $e\left(p, q^{\prime}\right) T_{(p, q)} \subseteq T_{(p, q)}$, it suffices to check that

$$
\bar{w} \beta_{p, q} L \subseteq \beta_{p, q} L
$$


where $\bar{w}$ is a monomial in $\bar{\alpha}_{p, q^{\prime \prime}}\left(q^{\prime \prime} \in \mathrm{L}(p)\right)$. Observe that relation (2.9) implies that $\bar{\alpha}_{p, q_{\ell}} \beta_{p, q_{j}}=\beta_{p, q_{j}} t_{\sigma_{j}(\ell)}^{-1}$ for $j \neq \ell$, and that $\bar{\alpha}_{p, q} \beta_{p, q}=0$. We have therefore shown that $e\left(p, q^{\prime}\right) T_{(p, q)} \subseteq T_{(p, q)}$. Now it follows from (2.21) and the above that $\bar{\alpha}_{p, q^{\prime}} T_{(p, q)} \subseteq T_{(p, q)}$, completing the proof of (1).

(2) This follows by "conjugating" the above relations and the following identity, for $f \in \Sigma(p)$, which comes from (2.20) and (2.16), (2.17), (2.18):

$$
\begin{aligned}
\bar{\beta}_{p, q_{j}} f^{-1}=\bar{\beta}_{p, q_{j}} e\left(p, q_{j}\right) f^{-1} & =\bar{\beta}_{p, q_{j}}\left(f_{0}^{\prime}\right)^{-1} \bar{w} e\left(p, q_{j}\right) \\
& =\left(\sigma^{p}\left(f_{0}^{\prime}\right)\left(t_{\sigma_{j}(\ell)}\right)\right)^{-1} w\left(t_{\sigma_{j}(\ell)}^{-1}\right) \bar{\beta}_{p, q_{j}},
\end{aligned}
$$

where $w$ is a monomial in $\left\{\alpha_{p, q_{\ell}}: \ell \neq j\right\}, f_{0}^{\prime} \in L\left[\alpha_{p, q_{\ell}}: \ell \neq j\right] \cap \Sigma(p)$, and $\sigma^{p}\left(f_{0}^{\prime}\right)\left(t_{\sigma_{j}(\ell)}\right) \in L$ is the polynomial obtained by applying $\sigma^{p}$ to all the coefficients of $f_{0}^{\prime}$ and replacing $\alpha_{p, q_{\ell}}$ with $t_{\sigma_{j}(\ell)}$, for $\ell \neq j$.

(3) and (4) follow from (2) and the relations (2.14), (2.9).

Let $\mathfrak{m}=z_{1}^{a_{1}} \cdots z_{k}^{a_{k}}$ be a commutative monomial in $z_{1}^{ \pm}, \ldots, z_{k}^{ \pm}$, so that $a_{i} \in \mathbb{Z}$. Assume that $p \in \mathbb{P}$ and that $\mathrm{L}(p)=\left\{q_{1}, \ldots, q_{k}\right\}$. We denote by $\mathfrak{m}\left(\alpha_{p, q_{1}}, \ldots, \alpha_{p, q_{k}}\right)$ the element of $Q_{K}(\mathbb{P})$ given by formally substituting $z_{i}^{a_{i}}$ by $\alpha_{p, q_{i}}^{a_{i}}$ if $a_{i}>0$, by $\left(\bar{\alpha}_{p, q_{i}}\right)^{-a_{i}}$ if $a_{i}<0$ and by $e(p)$ if $a_{i}=0$. We say that $\mathfrak{m}\left(\alpha_{p, q_{1}}, \ldots, \alpha_{p, q_{k}}\right)$ is a monomial in $\alpha_{p, q_{1}}, \ldots, \alpha_{p, q_{k}}, \bar{\alpha}_{p, q_{1}}, \ldots, \bar{\alpha}_{p, q_{k}}$. Observe that it does not depend on the way we order the elements $q_{1}, \ldots, q_{k}$.

Let $\mathcal{T}:=\mathcal{T}(\mathbb{P})$ be the quiver associated to the poset $\mathbb{P}$; see Definition 2.1. A path in $\mathcal{T}(\mathbb{P})$ is a sequence $\left(p_{1}, \ldots, p_{u}\right)$ of elements in $\mathbb{P}$ such that $p_{i+1} \in \mathrm{L}\left(p_{i}\right)$ for all $i$.

Lemma 2.11. Every element in $Q_{K}(\mathbb{P})$ can be written as a finite sum of elements of the form:

$$
\begin{aligned}
\left(f_{1}\right)^{-1} \alpha_{p_{1}, p_{2}}^{m_{1}} \beta_{p_{1}, p_{2}} & \cdots\left(f_{u-1}\right)^{-1} \alpha_{p_{u-1}, p_{u}}^{m_{u}} \beta_{p_{u-1}, p_{u}} \\
\cdot & \mathfrak{m}\left(\alpha_{p_{u}, q_{1}}, \ldots, \alpha_{p_{u}, q_{k}}\right)\left(f_{u}\right)^{-1} \\
& \bar{\beta}_{p_{u+1}, p_{u}} \bar{\alpha}_{p_{u+1}, p_{u}}^{m_{u}} \bar{\beta}_{p_{u+2}, p_{u+1}} \cdots \bar{\beta}_{p_{u+v+1}, p_{u+v}} \bar{\alpha}_{p_{u+v+1}, p_{u+v}}^{m_{u+v}}
\end{aligned}
$$

with $f_{i} \in \Sigma\left(p_{i}\right)$ for $i=1, \ldots, u, p_{i+1} \in \mathrm{L}\left(p_{i}\right)$ for all $i=1, \ldots, u-1$ and $p_{u+i} \in$ $\mathrm{L}\left(p_{u+i+1}\right)$ for all $i=1, \ldots, v$. Moreover $\mathfrak{m}\left(\alpha_{p_{u}, q_{1}}, \ldots, \alpha_{p_{u}, q_{k}}\right)$ is a monomial in $\alpha_{p_{u}, q_{1}}, \ldots, \alpha_{p_{u}, q_{k}}, \bar{\alpha}_{p_{u}, q_{1}}, \ldots, \bar{\alpha}_{p_{u}, q_{k}}$, where $\mathrm{L}\left(p_{u}\right)=\left\{q_{1}, \ldots, q_{k}\right\}$. We have

$$
Q_{K}(\mathbb{P})=\bigoplus_{\left(\gamma_{1}, \gamma_{2}\right) \in \mathcal{T}^{2}: r\left(\gamma_{1}\right)=r\left(\gamma_{2}\right)} Q_{K}(\mathbb{P})_{\left(\gamma_{1}, \gamma_{2}\right)},
$$

where $Q_{K}(\mathbb{P})_{\left(\gamma_{1}, \gamma_{2}\right)}$ is the L-vector space generated by all terms $\left({ }^{*}\right)$ with $\gamma_{1}=$ $\left(p_{1}, \ldots, p_{u}\right)$ and $\gamma_{2}=\left(p_{u+v+1}, \ldots, p_{u}\right)$.

Remark 2.12. Observe that if we have finitely many elements in $Q_{K}(\mathbb{P})_{\left(\gamma_{1}, \gamma_{2}\right)}$, we can express them as a finite sum of terms of the form $(*)$ where the sequence $\left(f_{1}, \ldots, f_{u-1}\right)$ is the same in all the terms. This can be done by taking common denominators, starting with the corresponding polynomials in $\Sigma\left(p_{1}\right)$, and then using relations (2.9) to rewrite all the terms with a common $f_{1}$. Now take common denominators for the polynomials from $\Sigma\left(p_{2}\right)$ appearing in the new terms, and rewrite again. After $u-1$ steps all the terms have the same sequence $\left(f_{1}, \ldots, f_{u-1}\right)$. We will refer to this process as "taking common denominators".

Proof of Lemma 2.11. The fact that $Q_{K}(\mathbb{P})=\sum_{\left(\gamma_{1}, \gamma_{2}\right) \in \mathcal{T}^{2}: r\left(\gamma_{1}\right)=r\left(\gamma_{2}\right)} Q_{K}(\mathbb{P})_{\left(\gamma_{1}, \gamma_{2}\right)}$ follows from Lemma 2.10, (Observe that the idempotents $e(p, q)$ can be replaced with $\beta_{p, q} \bar{\beta}_{p, q}$.) 
We have to prove that this is a direct sum. First we claim that the set $Q_{K}(\mathbb{P})_{(p, p)}$ of $L$-linear combinations of terms of the form $\mathfrak{m}\left(\alpha_{p, q_{1}}, \ldots, \alpha_{p, q_{k}}\right) f^{-1}$, where $f \in$ $\Sigma(p)$, and $\mathfrak{m}$ is a monomial in $\alpha_{p, q_{1}}, \ldots, \bar{\alpha}_{p, q_{k}}$, has trivial intersection with the ideal $J$ of $Q_{K}(\mathbb{P})$ generated by $e\left(q_{1}\right), \ldots, e\left(q_{k}\right)$. Indeed the canonical projection $Q_{K}(\mathbb{P}) \rightarrow Q_{K}(\mathbb{P}) / J$ induces an isomorphism $(e(p)+J)\left(Q_{K}(\mathbb{P}) / J\right)(e(p)+J) \cong$ $e(p) L\left(z_{1}, \ldots, z_{k}\right)$. The field $L\left(z_{1}, \ldots, z_{k}\right)$ is the directed union of the sets $L_{f}$ of elements of the form $\left(\sum_{i=1}^{s} \lambda_{i} \mathfrak{m}_{i}\right) f^{-1}$, where $\lambda_{i} \in L, \mathfrak{m}_{i}$ are (commutative) monomials in $z_{1}^{ \pm}, \ldots, z_{k}^{ \pm}$and $f \in L\left[z_{1}, \ldots, z_{k}\right]$ with $v(f)=0$. The $L$-linear map $e(p) L\left(z_{1}, \ldots, z_{k}\right) \rightarrow e(p) Q_{K}(\mathbb{P}) e(p)$ given by

$$
e(p) \mathfrak{m} f^{-1} \mapsto \mathfrak{m}\left(\alpha_{p, q_{1}}, \ldots, \alpha_{p, q_{k}}\right) f\left(\alpha_{p, q_{1}}, \ldots, \alpha_{p, q_{k}}\right)^{-1}
$$

is well defined due to the relations (2.5) and $\bar{\alpha}_{p, q_{i}} \alpha_{p, q_{i}}=e(p)$ for all $i$. This map provides a set-theoretical section of the projection $e(p) Q_{K}(\mathbb{P}) e(p) \rightarrow L\left(z_{1}, \ldots, z_{k}\right)$. This shows our claim.

Given a nonzero element $x$ in $Q_{K}(\mathbb{P})_{\left(\gamma, \gamma^{\prime}\right)}$, with $\gamma, \gamma^{\prime} \in \mathcal{T}(A)$ and $r(\gamma)=r\left(\gamma^{\prime}\right)$, written as a finite sum of elements of the form $\left(^{*}\right)$, we can take common denominators (Remark 2.12) and assume that every summand of the form $(*)$ has exactly the same sequence $\left(f_{1}, \ldots, f_{u-1}\right)$. Let $M_{1}$ be the highest power of $\alpha_{p_{1}, p_{2}}$ appearing in $x$, that is, such that $\bar{\alpha}_{p_{1}, p_{2}}^{M_{1}} f_{1} x \neq 0$. Then

$$
0 \neq \bar{\beta}_{p_{1}, p_{2}} \bar{\alpha}_{p_{1}, p_{2}}^{M_{1}} f_{1} x \in Q_{K}(\mathbb{P})_{\left(\gamma_{2}, \gamma^{\prime}\right)},
$$

where $\gamma_{2}=\left(p_{2}, \ldots, p_{u}\right)$. Proceeding in this way we see that there is

$$
z_{1}=\bar{\beta}_{p_{u-1}, p_{u}} \bar{\alpha}_{p_{1}, p_{2}}^{M_{u-1}} f_{u-1} \cdots \bar{\beta}_{p_{1}, p_{2}} \bar{\alpha}_{p_{1}, p_{2}}^{M_{1}} f_{1}
$$

and

$$
z_{2}=\alpha_{p_{u+v+1}, p_{u+v}}^{M_{u+v}} \beta_{p_{u+v+1}, p_{u+v}} \cdots \alpha_{p_{u+1}, p_{u}}^{M_{u}} \beta_{p_{u+1}, p_{u}}
$$

such that $z_{1} x z_{2} \in Q_{K}(\mathbb{P})_{\left(p_{u}, p_{u}\right)} \backslash\{0\}$.

Consider the following partial order on the set of pairs $\Gamma:=\left\{\left(\gamma, \gamma^{\prime}\right) \in \mathcal{T}^{2}: r(\gamma)=\right.$ $\left.r\left(\gamma^{\prime}\right)\right\}$ : say that $\left(\gamma_{1}, \gamma_{1}^{\prime}\right) \succeq\left(\gamma_{2}, \gamma_{2}^{\prime}\right)$ iff $\gamma_{2}=\gamma_{1} \gamma_{3}$ and $\gamma_{2}^{\prime}=\gamma_{1}^{\prime} \gamma_{3}^{\prime}$ for some paths $\gamma_{3}, \gamma_{3}^{\prime}$ in $\mathcal{T}$. In order to show that the sum in (2.22) is a direct sum, suppose that $\sum_{i=1}^{s} x_{\left(\gamma_{i}, \gamma_{i}^{\prime}\right)}=0$, with $\left(\gamma_{i}, \gamma_{i}^{\prime}\right)$ distinct elements of $\Gamma$ and $x_{\left(\gamma_{i}, \gamma_{i}^{\prime}\right)} \in Q_{K}(\mathbb{P})_{\left(\gamma_{i}, \gamma_{i}^{\prime}\right)} \backslash\{0\}$ for all $i$. We can assume that $\left(\gamma_{1}, \gamma_{1}^{\prime}\right)$ is maximal with respect to $\succeq$ (amongst the pairs $\left.\left(\gamma_{i}, \gamma_{i}^{\prime}\right)\right)$.

Set $p=r\left(\gamma_{1}\right)=r\left(\gamma_{1}^{\prime}\right)$. Let $z_{1}$ and $z_{2}$ be the elements of $Q_{K}(\mathbb{P})$ corresponding to $x_{\left(\gamma_{1}, \gamma_{1}^{\prime}\right)}$, constructed above, so that $z_{1} x_{\left(\gamma_{1}, \gamma_{1}^{\prime}\right)} z_{2} \in Q_{K}(\mathbb{P})_{(p, p)} \backslash\{0\}$. Observe that, by maximality of $\left(\gamma_{1}, \gamma_{1}^{\prime}\right)$, we have that $z_{1} Q_{K}(\mathbb{P})_{\left(\gamma_{i}, \gamma_{i}^{\prime}\right)} z_{2}=0$ unless $\left(\gamma_{i}, \gamma_{i}^{\prime}\right) \preceq\left(\gamma_{1}, \gamma_{1}^{\prime}\right)$. We conclude that

$$
\sum_{i=2}^{s} z_{1} Q_{K}(\mathbb{P})_{\left(\gamma_{i}, \gamma_{i}^{\prime}\right)} z_{2} \subseteq J
$$

where $J$ is the ideal of $Q_{K}(\mathbb{P})$ generated by $e\left(q_{1}\right), \ldots, e\left(q_{k}\right)$ with $\mathrm{L}(p)=\left\{q_{1}, \ldots, q_{k}\right\}$. We get

$$
0 \neq z_{1} x_{\left(\gamma_{1}, \gamma_{1}^{\prime}\right)} z_{2}=-\sum_{i=2}^{s} z_{1} x_{\left(\gamma_{i}, \gamma_{i}^{\prime}\right)} z_{2} \in Q_{K}(\mathbb{P})_{(p, p)} \cap J=0,
$$

which is a contradiction.

The method of proof of the above proposition gives the following nice technical lemma, which will be used to prove injectivity of some maps defined from $Q_{K}(\mathbb{P})$ to other $K$-algebras. For $x \in Q_{K}(\mathbb{P})$, write $x=\sum x_{\left(\gamma_{1}, \gamma_{2}\right)} \in \bigoplus_{r\left(\gamma_{1}\right)=r\left(\gamma_{2}\right)} Q_{K}(\mathbb{P})_{\left(\gamma_{1}, \gamma_{2}\right)}$; 
see Lemma2.11, Then the support of $x$ is the set of pairs $\left(\gamma_{1}, \gamma_{2}\right)$ such that $x_{\left(\gamma_{1}, \gamma_{2}\right)} \neq$ 0 .

Lemma 2.13. Assume that $x$ is a nonzero element in $Q_{K}(M(\mathbb{P}))$. Then there exist $p \in \mathbb{P}$ and $z_{1}, z_{2} \in Q_{K}(\mathbb{P})$ such that $z_{1} x z_{2}$ has the trivial pair of paths $(p, p)$ in the support, and all the other elements in the support of $z_{1} x z_{2}$ are of the form $\left(\gamma_{1}, \gamma_{2}\right)$, where $\gamma_{1}$ and $\gamma_{2}$ are paths in $\mathcal{T}$ starting at $p$ and ending in a common vertex.

Proof. Consider the partial order $\preceq$ of the proof of Proposition 2.11 on the support of $x$. Let $\left(\gamma_{1}, \gamma_{2}\right)$ be a maximal element of the support of $x$ with respect to this partial order, and set $p=r\left(\gamma_{1}\right)=r\left(\gamma_{2}\right)$. Let $z_{1}$ and $z_{2}$ be the elements of $Q_{K}(\mathbb{P})$ corresponding to $x_{\left(\gamma_{1}, \gamma_{2}\right)}$, constructed as in the proof of Lemma 2.11, Then $z_{1} x z_{2}$ has the desired properties.

In order to show the coherence of our construction (in particular to show that $e(p) \neq 0$ for every $p \in \mathbb{P}$ ), we will introduce a canonical faithful representation of the algebras $Q_{K}(\mathbb{P})$. Observe that for the simple case where $\mathbb{P}=\left\{p_{0}, p_{1}\right\}$ with $p_{0}<p_{1}$ our representation of $Q_{K}(\mathbb{P})$ is basically the canonical representation as Toeplitz operators on $L[z]$.

For a lower subset $A$ of $\mathbb{P}$, we will consider the idempotent

$$
e(A)=\sum_{q \in A} e(q) \in Q_{K}(\mathbb{P}) .
$$

Theorem 2.14. The algebra $Q_{K}(\mathbb{P})$ acts faithfully as $K$-linear maps on an $L$ vector space

$$
V(\mathbb{P})=\bigoplus_{p \in \mathbb{P}} V_{\mathbb{P}}(p),
$$

where $e(p)$ is the identity on $V(p)$ and 0 on $V(q)$ for $q \neq p$. If $A$ is a lower subset of $\mathbb{P}$, then we have a canonical isomorphism $\psi_{A}: Q_{K}(A) \longrightarrow e(A) Q_{K}(\mathbb{P}) e(A)$, and $V(A)=\bigoplus_{p \in A} V_{\mathbb{P}}(p)$. Moreover $(v) \psi_{A}(x)=(v) x$ for all $x \in Q_{K}(A)$ and all $v \in V(A)$.

Proof. We will define a right action of $Q_{K}(M)$ as $K$-linear endomorphisms on an $L$-vector space.

We proceed to build the $L$-vector spaces by induction. Set $\mathbb{P}^{0}:=\operatorname{Min}(\mathbb{P})$, the set of minimal elements of $\mathbb{P}$. If $\mathbb{P}^{0}, \ldots, \mathbb{P}^{i}$ have been defined, set $\mathbb{P}^{i+1}=$ $\operatorname{Min}\left(\mathbb{P} \backslash \bigcup_{j=0}^{i} \mathbb{P}^{j}\right)$. Obviously $\mathbb{P}=\bigcup_{i=0}^{r} \mathbb{P}^{i}$ for some $r$, and $\mathrm{L}(p) \subseteq \bigcup_{j=0}^{i} \mathbb{P}^{j}$ for $p \in \mathbb{P}^{i+1}$. Note that the sets $\bigcup_{j=0}^{i} \mathbb{P}^{j}$ are lower subsets of $\mathbb{P}$.

For $p \in \mathbb{P}^{0}$, set $V(p)=L$. The action of $e(p) Q_{K}\left(\mathbb{P}^{0}\right) e(p)=L$ on $V(p)=L$ is given by multiplication.

Now assume that the $L$-vector spaces $V(p)$ have been constructed for all $p \in$ $\mathbb{P}^{j}, 0 \leq j \leq i$, and that a representation $\tau_{i}: Q_{K}\left(\bigcup_{j=0}^{i} \mathbb{P}^{j}\right) \rightarrow \bigoplus_{p \in \cup_{j=0}^{i} \mathbb{P}^{j}} V(p)$ satisfying the required conditions has been defined. Let $p \in \mathbb{P}^{i+1}$ and write $\mathrm{L}(p)=$ $\left\{q_{1}, \ldots, q_{k}\right\}$. Observe that $V\left(q_{j}\right)$ has been defined. Now put

$$
V(p)=\bigoplus_{j=0}^{k} V\left(q_{j}\right) \otimes_{L} L_{j},
$$


where $L_{j}:=L\left(z_{1}, \ldots, z_{j-1}, z_{j+1}, \ldots, z_{k}\right)\left[z_{j}\right]$. Now the action of $\bar{\alpha}_{p, q_{\ell}}$ on $V\left(q_{j}\right) \otimes_{L} L_{j}$ is given by multiplication by $z_{\ell}$; that is,

$$
\left(v_{j} \otimes \lambda_{j}\right) \tau_{i+1}\left(\bar{\alpha}_{p, q_{\ell}}\right)=v_{j} \otimes \lambda_{j} z_{\ell}
$$

for $v_{j} \in V\left(q_{j}\right)$ and $\lambda_{j} \in L_{j}$. The action of $\alpha_{p, q_{\ell}}$ on $V\left(q_{j}\right) \otimes_{L} L_{j}$, with $j \neq \ell$, is given by

$$
\left(v_{j} \otimes \lambda_{j}\right) \tau_{i+1}\left(\alpha_{p, q_{\ell}}\right)=v_{j} \otimes \lambda_{j} z_{\ell}^{-1} .
$$

The action of $\alpha_{p, q_{\ell}}$ on $V\left(q_{\ell}\right) \otimes L_{\ell}$ is given as follows:

$$
\left(v_{\ell} \otimes\left(f_{0}+f_{1} z_{\ell}+\cdots+f_{v}\left(z_{\ell}\right)^{v}\right)\right) \tau_{i+1}\left(\alpha_{p, q_{\ell}}\right)=v_{\ell} \otimes\left(f_{1}+\cdots+f_{v}\left(z_{\ell}\right)^{v-1}\right),
$$

where $f_{b} \in L\left(z_{1}, \ldots, z_{\ell-1}, z_{\ell+1}, \ldots, z_{k}\right)$. Write $K_{j}=L\left(z_{1}, \ldots, z_{j-1}, z_{j+1}, \ldots, z_{k}\right)$. Note that $e\left(p, q_{j}\right)=e(p)-\alpha_{p, q_{j}} \bar{\alpha}_{p, q_{j}}$ projects $V(p)$ onto $V\left(q_{j}\right) \otimes_{L} K_{j}$. Thus define $\tau_{i+1}\left(\beta_{p, q_{j}}\right)$ as 0 on $\left(\bigoplus_{q \in \cup_{j=0}^{i+1} \mathbb{P}^{j}} V(q)\right)\left(1-e\left(p, q_{j}\right)\right)$, and as the isomorphism from $V\left(q_{j}\right) \otimes_{L} K_{j}$ onto $V\left(q_{j}\right)$ given by the composition

$$
V\left(q_{j}\right) \otimes_{L} L\left(z_{1}, \ldots, z_{j-1}, z_{j+1}, \ldots, z_{k}\right) \stackrel{1 \otimes \sigma^{j}}{\longrightarrow} V\left(q_{j}\right) \otimes_{L} L \stackrel{\cong}{\longrightarrow} V\left(q_{j}\right),
$$

where $\sigma^{j}$ is the isomorphism given by $\left(z_{\ell}\right) \sigma^{j}=t_{\sigma_{j}(\ell)}$ and $\left(t_{u}\right) \sigma^{j}=t_{u+k-1}$. The action of $\bar{\beta}_{p, q_{j}}$ is now determined by the rules (2.14).

It is straightforward to show that $\tau_{i+1}$ preserves the defining relations for the algebra $\mathcal{A} \Sigma_{1}^{-1}$ (see Definition 2.1). We have to check that $\tau_{i+1}(f)$ is an invertible endomorphism of $V(p)$ for each $f \in \Sigma(p)$. Let us fix $j$ with $1 \leq j \leq k$, and write

$$
f=f_{0}\left(\alpha_{p, q}\right)+\alpha_{p, q_{j}} f_{1}\left(\alpha_{p, q}\right)+\cdots+\left(\alpha_{p, q_{j}}\right)^{u} f_{u}\left(\alpha_{p, q}\right),
$$

where $f_{b} \in L\left[z_{1}, \ldots, z_{j-1}, z_{j+1}, \ldots, z_{k}\right]$. Let $g \mapsto \bar{g}$ denote the involution on $K_{j}$ which is the identity on $L$, and sends $z_{\ell}$ to $z_{\ell}^{-1}$ for $\ell \neq j$. Let $\phi_{j} \in \operatorname{End}_{K_{j}}\left(L_{j}\right)$ denote the map defined by

$$
\left(f_{0}+f_{1} z_{j}+\cdots+f_{v} z_{j}^{v}\right) \phi_{j}=f_{1}+\cdots+f_{v} z_{j}^{v-1},
$$

for $f_{b} \in K_{j}$. Then $\tau_{i+1}(f)$ acts on $V\left(q_{j}\right) \otimes_{L} L_{j}$ by

$$
\left(v_{j} \otimes \lambda_{j}\right) \tau_{i+1}(f)=v_{j} \otimes\left[\lambda_{j} \bar{f}_{0}+\left(\lambda_{j} \bar{f}_{1}\right) \phi_{j}+\cdots+\left(\lambda_{j} \bar{f}_{u}\right) \phi_{j}^{u}\right] .
$$

Since $\bar{f}_{0}+\bar{f}_{1} z_{j}+\cdots+\bar{f}_{u} z_{j}^{u} \in K_{j}\left[z_{j}\right]$ has constant term $\bar{f}_{0} \neq 0$ (because $f \in \Sigma(p)$ ), it is invertible in $K_{j}\left[\left[z_{j}\right]\right]$. Let

$$
G=\sum_{a=0}^{\infty} g_{a} z_{j}^{a} \in K_{j}\left[\left[z_{j}\right]\right]
$$

be the inverse of $\bar{f}_{0}+\bar{f}_{1} z_{j}+\cdots+\bar{f}_{u} z_{j}^{u}$. Then it is readily seen that $\sum_{a=0}^{\infty} g_{a} \phi_{j}^{a}$ defines an endomorphism on $V\left(q_{j}\right) \otimes_{L} L_{j}$ which is the inverse of the restriction of $\tau_{i+1}(f)$ to $V\left(q_{j}\right) \otimes_{L} L_{j}$. This shows that $\tau_{i+1}(f)$ is an invertible endomorphism of $V(p)$, as thus we obtain a representation $\tau_{i+1}: Q_{K}\left(\bigcup_{j=0}^{i+1} \mathbb{P}^{j}\right) \rightarrow \operatorname{End}_{K}\left(\bigoplus_{p \in \bigcup_{j=0}^{i+1} \mathbb{P}^{j}} V(p)\right)$. This completes the induction step.

In this way, we obtain a representation $\tau=\tau_{\mathbb{P}}: Q_{K}(\mathbb{P}) \rightarrow \operatorname{End}_{K}(V(\mathbb{P}))$. Observe that, if $A$ is a lower subset of $\mathbb{P}$, then $V(A)=\bigoplus_{p \in A} V_{\mathbb{P}}(p)$ and $\tau_{A}(x)=$ $\tau_{\mathbb{P}}\left(\psi_{A}(x)\right)_{\mid V(A)}$, where $\psi_{A}: Q_{K}(A) \rightarrow e(A) Q_{K}(\mathbb{P}) e(A)$ is the canonical map.

Now we are going to show that the representation $\tau$ is faithful. Assume that $x$ is a nonzero element of $Q_{K}(\mathbb{P})$. By Lemma 2.13, there are $z_{1}, z_{2}$ in $Q_{K}(\mathbb{P})$ such that $z_{1} x z_{2}$ has a nonzero coefficient in $Q_{K}(\mathbb{P})_{(p, p)}$ for some $p \in \mathbb{P}$, and all other nonzero coefficients of $z_{1} x z_{2}$ belong to $\sum Q_{K}(\mathbb{P})_{\left(\gamma_{1}, \gamma_{2}\right)}$, where in the above sum, $\left(\gamma_{1}, \gamma_{2}\right)$ 
ranges over all pairs of nontrivial paths starting in $p$ and ending in a common vertex. There are $f_{1}, f_{2} \in \Sigma(p)$ such that $f_{1}\left(z_{1} x z_{2}\right) f_{2}$ can be written as $y_{1}+y_{2}$ with $y_{1}=\sum_{i=1}^{s} \lambda_{i} \mathfrak{m}_{i} \in Q_{K}(\mathbb{P})_{(p, p)} \backslash\{0\}$ and $y_{2}=\sum_{u=1}^{n_{p}} \sum_{w=0}^{M_{u}} \alpha_{p, q_{u}}^{w} \beta_{p, q_{u}} y_{u w}$ for some $y_{u w} \in Q_{K}(\mathbb{P})$.

It is enough to show that $\tau\left(f_{1} z_{1} x z_{2} f_{2}\right) \neq 0$. Take a natural number $N$ such that $N$ is greater than $M_{1}$ and such that $N-n_{i}(1) \geq 0$ for all $i$, where $n_{i}(1)$ is the exponent of $z_{1}$ in the monomial $\mathfrak{m}_{i}(z)$. For a nonzero $v \in V\left(q_{1}\right)$, we consider the element $v \otimes z_{1}^{N}$ in $V\left(q_{1}\right) \otimes_{L} L_{1}$, and we compute

$\left(v \otimes z_{1}^{N}\right) \tau\left(f_{1} z_{1} x z_{2} f_{2}\right)=\left(v \otimes z_{1}^{N}\right) \tau\left(y_{1}\right)+\left(v \otimes z_{1}^{N}\right) \tau\left(y_{2}\right)=v \otimes\left(\sum_{i=1}^{s} \lambda_{i} z_{1}^{N} \overline{\mathfrak{m}}_{i}(z)\right) \neq 0$.

This shows that $\tau$ is faithful.

If $A$ is a lower subset of $\mathbb{P}$, then the map $\psi_{A}: Q_{K}(A) \rightarrow e(A) Q_{K}(\mathbb{P}) e(A)$ is surjective by Lemma 2.11 Since $\tau_{A}(x)=\tau_{\mathbb{P}}\left(\psi_{A}(x)\right)_{\mid V(A)}$ for $x \in Q_{K}(A)$, and $\tau_{A}$ is faithful, we conclude that $\psi_{A}$ is injective, and thus $\psi_{A}$ is an isomorphism.

The distributive lattice $\mathcal{L}(\mathbb{P})$ of lower subsets of $\mathbb{P}$, which agrees with the lattice $\mathcal{L}(M)$ of order-ideals of $M=M(\mathbb{P})$, can now be seen as a sublattice of $\mathcal{L}\left(Q_{K}(\mathbb{P})\right)$.

Proposition 2.15. Let $M$ be a finitely generated primitive monoid such that all primes of $M$ are free. Let $A \in \mathcal{L}(\mathbb{P}(M))$ be a lower subset of $\mathbb{P}(M)$ and consider the idempotent $e(A)=\sum_{q \in A} e(q) \in Q_{K}(\mathbb{P})$. Then the following properties hold:

(1) Let $I(A)$ be the ideal of $Q_{K}(\mathbb{P})$ generated by $e(A)$. Then

$$
I(A)=\bigoplus_{\left(\gamma_{1}, \gamma_{2}\right) \in \mathcal{T}^{2}: r\left(\gamma_{1}\right)=r\left(\gamma_{2}\right) \in A} Q_{K}(\mathbb{P})_{\left(\gamma_{1}, \gamma_{2}\right)} .
$$

(2) The assignment

$$
A \mapsto I(A)
$$

defines an injective lattice homomorphism from $\mathcal{L}(\mathbb{P})$ into $\mathcal{L}\left(Q_{K}(\mathbb{P})\right)$.

Proof. (1) This is clear from Lemma 2.11.

(2) It is clear from (1) that the map $\mathcal{L}(\mathbb{P}) \rightarrow \mathcal{L}\left(Q_{K}(\mathbb{P})\right)$ is injective. Observe that

$$
I(A)=\bigoplus_{a \in A} Q_{a}
$$

where $Q_{a}=\bigoplus_{\left(\gamma_{1}, \gamma_{2}\right) \in \mathcal{T}^{2}: r\left(\gamma_{1}\right)=r\left(\gamma_{2}\right)=a} Q_{K}(\mathbb{P})_{\left(\gamma_{1}, \gamma_{2}\right)}$. It follows that $I(A)+I(B)=$ $I(A \cup B)$ and $I(A) \cap I(B)=I(A \cap B)$. Thus the above map is a lattice homomorphism.

Remark 2.16. It follows from Theorem 2.3 and Propositions 1.1 and 1.2 that the map $\mathcal{L}(\mathbb{P}) \rightarrow \mathcal{L}\left(Q_{K}(\mathbb{P})\right)$ of Corollary 2.15)(2) is indeed a lattice isomorphism.

Remark 2.17. If $A$ is a lower subset of $\mathbb{P}$, then $Q_{K}(M(\mathbb{P})) / I(A)$ is not in general isomorphic with $Q_{K}(M(\mathbb{P} \backslash A))$. However the structure of $Q_{K}(M(\mathbb{P})) / I(A)$ is clear from the presentation given in Definition 2.1. Indeed the generators and relations for $Q_{K}(M(\mathbb{P})) / I(A)$ are the same as in 2.1 for all the primes $p \in \mathbb{P} \backslash A$ such that $\mathrm{L}(p) \cap A=\emptyset$, and if $p \in \mathbb{P} \backslash A$ and $\mathrm{L}(p) \cap A \neq \emptyset$, then one has to add additional relations $e(p, q)=\beta_{p, q}=0$ for $q \in \mathrm{L}(p) \cap A$. Of course we can omit in the presentation all idempotents $e(p)$ with $p \in A$, because these idempotents are 0 in $Q_{K}(M(\mathbb{P})) / I(A)$. 


\section{Pullbacks}

In this section we develop part of the machinery used in the proof of our main result, concerning pullbacks of von Neumann regular rings. It is important to have in mind that, although a pullback of regular rings is always regular, the corresponding diagram at the level of monoids of f.g. projectives might not be a pullback in the category of monoids. We give necessary and sufficient conditions in order for this property to hold in $K$-theoretic terms.

Proposition 3.1. Let $Q_{1}$ and $Q_{2}$ be two von Neumann regular (resp. exchange) rings and let $\pi_{i}: Q_{i} \rightarrow R$ be surjective homomorphisms. Consider the pullback

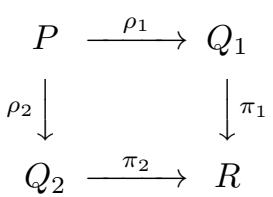

in the category of rings. Then $R$ and $P$ are von Neumann regular (resp. exchange) rings. If $Q_{1}$ and $Q_{2}$ are (strongly) separative, then $R$ and $P$ are (strongly) separative too.

Proof. Since $Q_{1}$ is a von Neumann regular (resp. exchange) ring and $\pi_{1}$ is surjective, $R$ is von Neumann regular (resp. exchange) ([24, Lemma 1.3], [30, Proposition 1.4]). Let $I_{i} \triangleleft Q_{i}$ be the kernel of $\pi_{i}, i=1,2$. We have a commutative diagram with exact rows

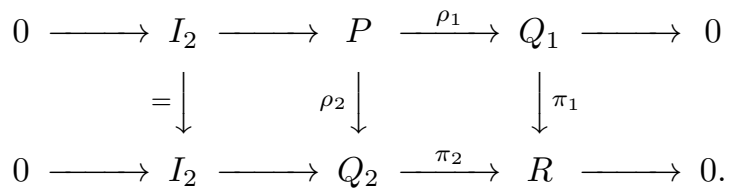

Assume that $Q_{1}$ and $Q_{2}$ are von Neumann regular. Then $I_{2}$ and $Q_{1}$ are von Neumann regular, and it follows from [24, Lemma 1.3] that $P$ is also von Neumann regular. If $Q_{1}$ and $Q_{2}$ are exchange rings, then $I_{2}$ and $Q_{1}$ are also exchange rings (see [5. Theorem 2.2]), and idempotents of $Q_{1}$ can be lifted to idempotents of $P$, because, $Q_{2}$ being an exchange ring, idempotents in $R$ can be lifted to idempotents in $Q_{2}$. It follows from [5, Theorem 2.2] that $P$ is an exchange ring.

If $Q_{1}$ and $Q_{2}$ are (strongly) separative, then $R$ is (strongly) separative because it is a factor ring of a (strongly) separative exchange ring. Also [11, Theorem 4.5] ([11, Theorem 5.2]) shows that $P$ is (strongly) separative.

Let $Q_{1}, \ldots, Q_{k}$ be rings, and let $\pi_{i}: Q_{i} \rightarrow R$ be surjective homomorphisms. Let $\rho_{i}: P \rightarrow Q_{i}$ be the limit (pullback) of the morphisms $\pi_{i}: Q_{i} \rightarrow R$. We have $k$ index maps $\partial_{i}: K_{1}(R) \rightarrow K_{0}\left(I_{i}\right)$ for $i=1, \ldots, k$, where $I_{i}$ is the kernel of $\pi_{i}$. If $e=\left(e_{1}, \ldots, e_{k}\right)$ is an idempotent in $P$, then we get corresponding maps $\partial_{i}: K_{1}\left(\pi_{i}\left(e_{i}\right) R \pi_{i}\left(e_{i}\right)\right) \rightarrow K_{0}\left(e_{i} I_{i} e_{i}\right)$. These maps fit into an exact sequence

$$
K_{1}\left(e_{i} Q_{i} e_{i}\right) \stackrel{\left(\pi_{i}\right)_{*}}{\longrightarrow} K_{1}\left(\pi_{i}\left(e_{i}\right) R \pi_{i}\left(e_{i}\right)\right) \stackrel{\partial_{i}}{\longrightarrow} K_{0}\left(e_{i} I_{i} e_{i}\right) \longrightarrow K_{0}\left(e_{i} Q_{i} e_{i}\right) .
$$

We are now ready to state the main result of this section. Note that the $K$ theoretic part of the result below might be considered as a nonstable version of [29, Theorem 3.3].

Theorem 3.2. Let $Q_{1}, \ldots, Q_{k}$ be (strongly) separative von Neumann regular (resp. exchange) rings, and let $\pi_{i}: Q_{i} \rightarrow R$ be surjective homomorphisms. Let $\rho_{i}: P \rightarrow Q_{i}$ 
be the limit (pullback) of the morphisms $\pi_{i}: Q_{i} \rightarrow R$. Then $P$ is a (strongly) separative von Neumann regular (resp. exchange) ring, and the maps $\mathcal{V}\left(\rho_{i}\right): \mathcal{V}(P) \rightarrow$ $\mathcal{V}\left(Q_{i}\right)$ are the limit of the family of maps $\mathcal{V}\left(\pi_{i}\right): \mathcal{V}\left(Q_{i}\right) \rightarrow \mathcal{V}(R)$ in the category of monoids if and only if for each idempotent $e=\left(e_{1}, \ldots, e_{k}\right)$ in $P$, we have that for every $i=1, \ldots, k$,

$$
K_{1}\left(\pi_{i}\left(e_{i}\right) R \pi_{i}\left(e_{i}\right)\right)=\left(\pi_{i}\right)_{*}\left(K_{1}\left(e_{i} Q_{i} e_{i}\right)\right)+\left(\bigcap_{j \neq i}\left(\pi_{j}\right)_{*}\left(K_{1}\left(e_{j} Q_{j} e_{j}\right)\right)\right) .
$$

It is enough to check the above condition for some generator $e=\left(e_{1}, \ldots, e_{k}\right)$ of each finitely generated trace ideal of $P$.

Proof. The first part follows easily by induction from Proposition 3.1

Assume now that $Q_{1}, \ldots, Q_{k}$ are just exchange rings. (The separativity will be used later in the proof.) Let $\tau_{i}: M \rightarrow \mathcal{V}\left(Q_{i}\right)$ be the limit of the family of maps $\mathcal{V}\left(\pi_{i}\right): \mathcal{V}\left(Q_{i}\right) \rightarrow \mathcal{V}(R)$ in the category of monoids. Recall that

$$
M=\left\{\left(x_{1}, \ldots, x_{n}\right) \in \prod \mathcal{V}\left(Q_{i}\right): \mathcal{V}\left(\pi_{i}\right)\left(x_{i}\right)=\mathcal{V}\left(\pi_{j}\right)\left(x_{j}\right), \text { for all } i, j\right\}
$$

Obviously we have a unique monoid homomorphism $\rho: \mathcal{V}(P) \rightarrow M$ such that $\tau_{i} \circ \rho=$ $\mathcal{V}\left(\rho_{i}\right)$ for all $i$. We show that the map $\rho$ is always surjective. (We don't need the extra condition in the statement to prove this.) Let $P^{\prime}$ be the limit of the family of maps $\pi_{i}: Q_{i} \rightarrow R$, for $1 \leq i \leq k-1$, and let $M^{\prime}$ be the limit of the family $\mathcal{V}\left(\pi_{i}\right): \mathcal{V}\left(Q_{i}\right) \rightarrow \mathcal{V}(R), 1 \leq i \leq k-1$. Let $\rho^{\prime}: \mathcal{V}\left(P^{\prime}\right) \rightarrow M^{\prime}$ be the canonical map. Assume that $\left(\left[e_{1}\right],\left[e_{2}\right], \ldots,\left[e_{k}\right]\right)$ is an element in $M$, with $e_{i} \in \operatorname{Idem}\left(M_{n}\left(Q_{i}\right)\right)$ for some $n \geq 1$. By induction, there is an $f \in M_{m}\left(P^{\prime}\right)$ for some $m \geq n$ such that

$$
\rho^{\prime}([f])=\left(\left[e_{1}\right], \ldots,\left[e_{k-1}\right]\right) \in M^{\prime} .
$$

Replacing each $e_{i}$ with $e_{i} \oplus 0_{m-n}$, we can assume that all $e_{i} \in M_{m}\left(Q_{i}\right)$ for all $i$. Observe that

$$
\pi_{k}\left(e_{k}\right) \sim \pi_{1}\left(f_{1}\right)=\cdots=\pi_{k-1}\left(f_{k-1}\right),
$$

where $f=\left(f_{1}, \ldots, f_{k-1}\right)$. By [5, Lemma 3.1(a)], there exists an idempotent $q$ in $M_{m}\left(Q_{k}\right)$ such that $q \sim e_{k}-e_{k}^{\prime}$ for some idempotent $e_{k}^{\prime} \in e_{k} M_{m}\left(I_{k}\right) e_{k}$, and $\pi_{k}(q)=\pi_{1}\left(f_{1}\right)$. Consider the idempotent

$$
e=\left(f_{1} \oplus 0, \ldots, f_{k-1} \oplus 0, q \oplus e_{k}^{\prime}\right) \in \prod M_{2 m}\left(Q_{i}\right) .
$$

Then $\pi_{1}\left(f_{1} \oplus 0\right)=\cdots=\pi_{k-1}\left(f_{k-1} \oplus 0\right)=\pi_{k}\left(q \oplus e_{k}^{\prime}\right)$ so that $e \in M_{2 m}(P)$ and clearly $\rho([e])=\left(\left[e_{1}\right], \ldots,\left[e_{k}\right]\right)$, showing that $\rho$ is surjective.

Assume first that the maps $\mathcal{V}\left(\rho_{i}\right): \mathcal{V}(P) \rightarrow \mathcal{V}\left(Q_{i}\right)$ are the limit of the family of maps $\mathcal{V}\left(\pi_{i}\right): \mathcal{V}\left(Q_{i}\right) \rightarrow \mathcal{V}(R)$ in the category of monoids, and let us show (3.1). This implication does not use separativity.

Assume that $[u] \in K_{1}(R)$, with $u \in G L_{n}(R)$ for some $n \geq 1$. Then $\partial_{1}([u])=$ $[1-y x]-[1-x y]$, where $x, y \in M_{n}\left(Q_{1}\right)$ and $x=x y x, y=y x y$, and $\pi_{1}(x)=u$. (Such a lifting of $u$ exists by [5, Lemma 2.1].) Put $e_{1}=y x$ and $e_{1}^{\prime}=x y$. Then we have $\pi_{1}\left(e_{1}\right)=\pi_{1}\left(e_{1}^{\prime}\right)=1_{n}$. Consider the idempotents $\left(e_{1}, 1_{n}, \ldots, 1_{n}\right)$ and $\left(e_{1}^{\prime}, 1_{n}, \ldots, 1_{n}\right)$ in $M_{n}(P)$, where $1_{n}$ is the identity matrix of size $n \times n$. Clearly

$$
\rho\left(\left[\left(e_{1}, 1_{n}, \ldots, 1_{n}\right)\right]\right)=\rho\left(\left[\left(e_{1}^{\prime}, 1_{n}, \ldots, 1_{n}\right)\right]\right) \in M .
$$

Since $\rho$ is an isomorphism, we get $\left(e_{1}, 1_{n}, \ldots, 1_{n}\right) \sim\left(e_{1}^{\prime}, 1_{n}, \ldots, 1_{n}\right)$ in $M_{n}(P)$. We get elements

$$
\left(y_{1}, z_{2}, \ldots, z_{k}\right) \in\left(e_{1}, 1_{n}, \ldots, 1_{n}\right) P\left(e_{1}^{\prime}, 1_{n}, \ldots, 1_{n}\right)
$$


and

such that

$$
\left(x_{1}, t_{2}, \ldots, t_{k}\right) \in\left(e_{1}^{\prime}, 1_{n}, \ldots, 1_{n}\right) P\left(e_{1}, 1_{n}, \ldots, 1_{n}\right)
$$

and

$$
\left(y_{1}, z_{2}, \ldots, z_{k}\right)\left(x_{1}, t_{2}, \ldots, t_{k}\right)=\left(e_{1}, 1_{n}, \ldots, 1_{n}\right)
$$

$$
\left(x_{1}, t_{2}, \ldots, t_{k}\right)\left(y_{1}, z_{2}, \ldots, z_{k}\right)=\left(e_{1}^{\prime}, 1_{n}, \ldots, 1_{n}\right) .
$$

So we get that $t_{i} \in G L_{n}\left(Q_{i}\right)$ and $\pi_{1}\left(x_{1}\right)=\pi_{i}\left(t_{i}\right)$ for all $i \geq 2$, so that

$$
\left[\pi_{1}\left(x_{1}\right)\right] \in \bigcap_{i \neq 1}\left(\pi_{i}\right)_{*}\left(K_{1}\left(Q_{i}\right)\right) .
$$

Furthermore

$$
\partial_{1}\left(\left[\pi_{1}\left(x_{1}\right)\right]\right)=\left[1-y_{1} x_{1}\right]-\left[1-x_{1} y_{1}\right]=\left[1-e_{1}\right]-\left[1-e_{1}^{\prime}\right]=\partial_{1}([u]) .
$$

By exactness of the $K$-theory sequence, we get that $[u]-\left[\pi_{1}\left(x_{1}\right)\right] \in\left(\pi_{1}\right)_{*}\left(K_{1}\left(Q_{1}\right)\right)$, so that $[u] \in\left(\pi_{1}\right)_{*}\left(K_{1}\left(Q_{1}\right)\right)+\bigcap_{i \neq 1}\left(\pi_{i}\right)_{*}\left(K_{1}\left(Q_{i}\right)\right)$. This shows that

$$
K_{1}(R)=\left(\pi_{1}\right)_{*}\left(K_{1}\left(Q_{1}\right)\right)+\bigcap_{i \neq 1}\left(\pi_{i}\right)_{*}\left(K_{1}\left(Q_{i}\right)\right) .
$$

If $e=\left(e_{1}, \ldots, e_{k}\right)$ is an idempotent in $P$, then the maps $\rho_{i \mid}: e P e \rightarrow e_{i} Q_{i} e_{i}$ give the limit of the family of maps $\pi_{i \mid}: e_{i} Q_{i} e_{i} \rightarrow \pi_{i}\left(e_{i}\right) R \pi_{i}\left(e_{i}\right)$, and the maps $\mathcal{V}\left(\rho_{i \mid}\right): \mathcal{V}(e P e) \rightarrow \mathcal{V}\left(e_{i} Q_{i} e_{i}\right)$ give the limit of the family $\mathcal{V}\left(\pi_{i \mid}\right): \mathcal{V}\left(e_{i} Q_{i} e_{i}\right) \rightarrow$ $\mathcal{V}\left(\pi_{i}\left(e_{i}\right) R \pi_{i}\left(e_{i}\right)\right)$ in the category of monoids. (Observe that, if $e$ is an idempotent in a general ring $T$, then we can identify $\mathcal{V}(e T e)$ with the order-ideal $\mathcal{V}(T e T)$ of $\mathcal{V}(T)$ consisting of classes $[g] \in \mathcal{V}(R)$ such that $g \in M_{\infty}(T e T)$; see [9, proof of Lemma 7.3].) By the above argument, we get (3.1).

Now assume that (3.1) holds for every idempotent $e=\left(e_{1}, \ldots, e_{k}\right)$ in $P$ and every index $i$. Assume that $Q_{1}, \ldots, Q_{k}$ are separative exchange rings. Since $\rho: \mathcal{V}(P) \rightarrow$ $M$ is always surjective, we only have to show that it is injective. Let $\left(e_{1}, \ldots, e_{k}\right)$ and $\left(e_{1}^{\prime}, \ldots, e_{k}^{\prime}\right)$ be idempotents in $M_{n}(P)$ for some $n \geq 1$ such that $e_{i} \sim e_{i}^{\prime}$ in $M_{n}\left(Q_{i}\right)$ for all $i$. By using standard arguments, and passing to matrices of larger size, we can assume that $e_{k}=u_{k} e_{k}^{\prime} u_{k}^{-1}$, where $u_{k} \in E_{m}\left(Q_{k}\right)$, the group of elementary matrices of size $m \times m$ for some $m \geq n$; see [34, 1.2.1, 2.1.3]. Now since all elementary matrices lift, there is $u_{i} \in E_{m}\left(Q_{i}\right)$ such that $\pi_{i}\left(u_{i}\right)=\pi_{k}\left(u_{k}\right)$, so that $u:=\left(u_{1}, \ldots, u_{k-1}, u_{k}\right) \in G L_{m}(P)$ and $u\left(e_{1}^{\prime}, \ldots, e_{k}^{\prime}\right) u^{-1}=\left(e_{1}^{\prime \prime}, \ldots, e_{k-1}^{\prime \prime}, e_{k}\right)$, so we can assume that $e_{k}=e_{k}^{\prime}$.

Thus assume we have two idempotents $\left(e_{1}, \ldots, e_{k-1}, e_{k}\right)$ and $\left(e_{1}^{\prime}, \ldots, e_{k-1}^{\prime}, e_{k}\right)$ in $M_{n}(P)$ such that $e_{i} \sim e_{i}^{\prime}$ in $M_{n}\left(Q_{i}\right)$ for $i=1, \ldots, k-1$. We have $\pi_{i}\left(e_{i}\right)=\pi_{k}\left(e_{k}\right)=$ $\pi_{j}\left(e_{j}^{\prime}\right)$ for all $i, j$. Replacing each ring $T$ in the diagram with $M_{n}(T)$, we can assume that $n=1$. (The validity of (3.1) for idempotents in $M_{n}(P)$ is indeed justified by the last sentence in the statement: the trace ideal of $P$ generated by an idempotent $E$ in $M_{n}(P)$ is generated by a single idempotent of $P$; see the last paragraph in the proof.) By using induction, we can write $\left(e_{1}, \ldots, e_{k-1}\right)=x y$ and $\left(e_{1}^{\prime}, \ldots, e_{k-1}^{\prime}\right)=$ $y x$ with $x \in\left(e_{1}, \ldots, e_{k-1}\right) P^{\prime}\left(e_{1}^{\prime}, \ldots, e_{k-1}^{\prime}\right)$ and $y \in\left(e_{1}^{\prime}, \ldots, e_{k-1}^{\prime}\right) P^{\prime}\left(e_{1}, \ldots, e_{k-1}\right)$, where $P^{\prime}$ is the limit of the family of maps $\pi_{i}: Q_{i} \rightarrow R, 1 \leq i \leq k-1$. We have

$$
\pi_{1}\left(x_{1}\right) \pi_{1}\left(y_{1}\right)=\pi_{1}\left(e_{1}\right)=\pi_{1}\left(e_{1}^{\prime}\right)=\pi_{1}\left(y_{1}\right) \pi_{1}\left(x_{1}\right),
$$

so that $\pi_{1}\left(x_{1}\right)=\pi_{i}\left(x_{i}\right) \in G L_{1}\left(\pi_{1}\left(e_{1}\right) R \pi_{1}\left(e_{1}\right)\right)$, for $1 \leq i \leq k-1$, and $\pi_{1}\left(y_{1}\right)=$ $\pi_{1}\left(x_{1}\right)^{-1}$. Take $z_{i}, t_{i} \in e_{i} Q_{i} e_{i}, 1 \leq i \leq k-1$, such that $z_{i}=z_{i} t_{i} z_{i}$ and $t_{i}=$ $t_{i} z_{i} t_{i}$, with $\pi_{i}\left(z_{i}\right)=\pi_{i}\left(x_{i}\right)$, so that $\pi_{i}\left(t_{i}\right)=\pi_{i}\left(y_{i}\right)$. Set $z=\left(z_{1}, \ldots, z_{k-1}\right)$ and 
$t=\left(t_{1}, \ldots, t_{k-1}\right)$, and note that $z, t \in f P^{\prime} f$, where $f=\left(e_{1}, \ldots, e_{k-1}\right)$. Put $f^{\prime}=\left(e_{1}^{\prime}, \ldots, e_{k-1}^{\prime}\right) \in P^{\prime}$. Write $h:=z t$ and $h^{\prime}:=t z$. Then $h, h^{\prime} \in f P^{\prime} f$ and

$$
\left(f, e_{k}\right)=\left(h, e_{k}\right)+(f-h, 0)
$$

with $f-h \in \prod_{i=1}^{k-1} I_{i}$. On the other hand,

$$
\left(f^{\prime}, e_{k}\right)=\left(y h x, e_{k}\right)+\left(f^{\prime}-y h x, 0\right),
$$

and clearly $(f-h, 0) \sim\left(f^{\prime}-y h x, 0\right)$ in $P$, so it suffices to check that $\left(h, e_{k}\right) \sim$ $\left(y h x, e_{k}\right)$ in $P$. Let $\pi^{\prime}: P^{\prime} \rightarrow R$ be the canonical map. Since $\pi^{\prime}(y z)=\pi^{\prime}(t x)=$ $\pi_{k}\left(e_{k}\right)$, we get

$$
\left(y h x, e_{k}\right)=\left(y z, e_{k}\right)\left(h^{\prime}, e_{k}\right)\left(t x, e_{k}\right)
$$

so that $\left(y h x, e_{k}\right) \sim\left(h^{\prime}, e_{k}\right)$ in $P$.

Therefore we only have to show that $\left(h, e_{k}\right) \sim\left(h^{\prime}, e_{k}\right)$ in $P$. By applying our hypothesis (with the idempotent $\left(h, e_{k}\right)$ ), we can write $\left[\pi^{\prime}(z)\right]=\alpha+\beta$ in $K_{1}\left(\pi^{\prime}(h) R \pi^{\prime}(h)\right)$, where $\alpha \in\left(\pi_{k}\right)_{*}\left(K_{1}\left(e_{k} Q_{k} e_{k}\right)\right)$ and $\beta \in \bigcap_{i=1}^{k-1}\left(\pi_{i}\right)_{*}\left(K_{1}\left(h_{i} Q_{i} h_{i}\right)\right)$.

By [12, we can choose $v$ in $G L_{1}\left(e_{k} Q_{k} e_{k}\right)$ such that $\left(\pi_{k}\right)_{*}([v])=\alpha$, so that $\left[\pi^{\prime}(z) \pi_{k}(v)^{-1}\right]=\beta$. Since $\partial_{i}(\beta)=0$ for $1 \leq i \leq k-1$, it follows from [31, Theorem $2.4]$ that there exists a unit $u \in G L_{1}\left(h P^{\prime} h\right)$ such that $\pi^{\prime}(u)=\pi^{\prime}(z) \pi_{k}(v)^{-1}$, so that $\left(u^{-1} z, v\right) \in P$ and

$$
\left(u^{-1} z, v\right)\left(h^{\prime}, e_{k}\right)\left(t u, v^{-1}\right)=\left(u^{-1} z h^{\prime} t u, v e_{k} v^{-1}\right)=\left(u^{-1} h u, e_{k}\right)=\left(h, e_{k}\right)
$$

and

$$
\left(t u, v^{-1}\right)\left(h, e_{k}\right)\left(u^{-1} z, v\right)=\left(h^{\prime}, e_{k}\right)
$$

and we get that $\left(h^{\prime}, e_{k}\right) \sim\left(h, e_{k}\right)$ in $P$, as desired.

The last sentence in the statement of the theorem follows from the Morita invariance of $K_{1}$. Observe that, $P$ being an exchange ring, the finitely generated trace ideals of $P$ are the ideals generated by finitely many idempotents (cf. [9, page 377]) and so, by [12, Lemma 2.1], they are the ideals generated by a single idempotent.

Example 3.3. To illustrate Theorem 3.2 in the context of our construction, we check that the conditions in the theorem are satisfied for the pullback diagram (2.19) in Example 2.5 We may assume that $\left(e_{1}, e_{2}\right)=\left(1_{Q_{1}}, 1_{Q_{2}}\right)$. We have that $\left(\pi_{1}\right)_{*}\left(K_{1}\left(Q_{1}\right)\right)=\left(K\left(t_{2}, t_{3}, \ldots\right)\right)\left[t_{1}\right]_{\left(t_{1}\right)}$ and $\left(\pi_{2}\right)_{*}\left(K_{1}\left(Q_{2}\right)\right)=\left(K\left(t_{1}, t_{3}, \ldots\right)\right)\left[t_{2}\right]_{\left(t_{2}\right)}$, so that

$$
K_{1}(L)=L^{\times}=\left(\pi_{1}\right)_{*}\left(K_{1}\left(Q_{1}\right)\right)+\left(\pi_{2}\right)_{*}\left(K_{1}\left(Q_{2}\right)\right),
$$

as desired. Since $Q_{1}$ and $Q_{2}$ are strongly separative von Neumann regular rings (see [7, 4.3]), we conclude from Theorem 3.2 that $Q_{K}(\mathbb{P})$ is a strongly separative von Neumann regular ring and that $\mathcal{V}\left(Q_{K}(\mathbb{P})\right) \cong\langle p, a, b \mid p=p+a=p+b\rangle$ (cf. Proposition 4.6).

\section{Pushouts}

In this section we discuss some constructions of monoids and rings associated to a certain class of pushouts of monoids. The section also includes some needed computation on pullbacks (Proposition 4.6). We refer the reader to [23, Chapter 8] for the general theory of free products with amalgams of semigroups. 
Lemma 4.1. Let $M$ and $N$ be two conical monoids. Let $I$ be an order-ideal of $M$ which is isomorphic with an order-ideal of $N$, through an isomorphism $\varphi: I \rightarrow$ $\varphi(I) \triangleleft N$. Let $P$ be the monoid $M \times N / \sim$, where $\sim$ is the congruence on $M \times N$ generated by all pairs $((s, 0),(0, \varphi(s)))$ for $s \in I$. Then we have a pushout diagram

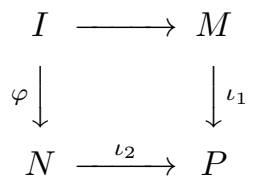

where $\iota_{1}: M \rightarrow P$ is the map $\iota_{1}(x)=[(x, 0)]$ and $\iota_{2}: N \rightarrow P$ is the map $\iota_{2}(y)=$ $[(0, y)]$. The maps $\iota_{1}$ and $\iota_{2}$ are injective and $I^{\prime}:=\iota_{1}(I)=\iota_{2}(\varphi(I))$ is an order-ideal of $P$ such that $P / I^{\prime} \cong M / I \times N / \varphi(I)$. Moreover, if there exist injective monoid homomorphisms $\theta_{1}: M \rightarrow Q$ and $\theta_{2}: N \rightarrow Q$ into a conical refinement monoid $Q$ such that $\theta_{1 \mid I}=\theta_{2} \circ \varphi$ and $\theta_{1}(M) \cap \theta_{2}(N)=\theta_{1}(I)=\theta_{2}(\varphi(I))$, and $\theta_{1}(M)$ and $\theta_{2}(N)$ are order-ideals of $Q$, then there is an embedding $\iota: P \rightarrow Q$ such that $\theta_{i}=\iota \circ \iota_{i}$ for $i=1,2$.

Proof. It is clear that the diagram is a pushout diagram. We will show that the map $\iota_{1}$ is injective. The proof for $\iota_{2}$ is similar. If $\left(s_{1}, 0\right) \sim\left(s_{2}, 0\right)$, then there are sequences $x_{i}, y_{i}$, with $y_{i} \in I$, such that $s_{1}=x_{1}+y_{1}, y_{1}=x_{2}+y_{2}$ and for all $i$, $x_{2 i-1}+x_{2 i}=x_{2 i+1}+y_{2 i+1}$, and $y_{2 i}+y_{2 i+1}=x_{2 i+2}+y_{2 i+2}$, such that

$$
\begin{gathered}
\left(s_{1}, 0\right)=\left(x_{1}+y_{1}, 0\right) \sim\left(x_{1}, \varphi\left(y_{1}\right)\right)=\left(x_{1}, \varphi\left(x_{2}+y_{2}\right)\right) \sim\left(x_{1}+x_{2}, \varphi\left(y_{2}\right)\right) \\
=\left(x_{3}+y_{3}, \varphi\left(y_{2}\right)\right) \sim \cdots \sim\left(s_{2}, 0\right) .
\end{gathered}
$$

Since $M$ is conical, there is an $n$ such that $y_{2 n}=0$ and $s_{2}=x_{2 n-1}+x_{2 n}$. We thus get

$$
s_{2}=x_{2 n-1}+x_{2 n}=x_{2 n-1}+y_{2 n-2}+y_{2 n-1}=\cdots=x_{1}+y_{1}=s_{1} .
$$

In general, note that $(s, 0) \sim(x, y)$, where $s \in M$ and $x \in M$ and $y \in N$, implies $y=\varphi\left(x^{\prime}\right)$ for some $x^{\prime} \in I$ such that $x+x^{\prime}=s$, so we get that $M$ and $I$ are order-ideals of $P$.

Since $P$ is the pushout of the diagram, we clearly have a map $\gamma^{\prime}: P \rightarrow M / I \times$ $N / \varphi(I)$, defined by $\gamma([(x, y)])=([x],[y])$. Clearly this map factors through a map $\gamma: P / I^{\prime} \rightarrow M / I \times N / \varphi(I)$. The inverse of this map is provided by the well-defined map $\tau: M / I \times N / \varphi(I) \rightarrow P / I^{\prime}$, given by $\tau([x],[y])=[(x, y)] \in P / I^{\prime}$.

Finally, assume that there exist injective monoid homomorphisms $\theta_{1}: M \rightarrow Q$ and $\theta_{2}: N \rightarrow Q$ into a conical refinement monoid $Q$ such that $\theta_{1 \mid I}=\theta_{2} \circ \varphi$ and $\theta_{1}(M) \cap \theta_{2}(N)=\theta_{1}(I)=\theta_{2}(\varphi(I))$, and $\theta_{1}(M)$ and $\theta_{2}(N)$ are order-ideals of $Q$. By the pushout property there is a monoid homomorphism $\iota: P \rightarrow Q$ such that $\theta_{i}=\iota \circ \iota_{i}$ for $i=1,2$. It remains to show that $\iota$ is injective. So assume that for $\left[\left(x_{1}, y_{1}\right)\right],\left[\left(x_{2}, y_{2}\right)\right] \in P$ we have $\theta_{1}\left(x_{1}\right)+\theta_{2}\left(y_{1}\right)=\theta_{1}\left(x_{2}\right)+\theta_{2}\left(y_{2}\right)$. Applying refinement in $Q$ we get $\theta_{1}\left(x_{1}\right)=z_{1}+z_{2}$ and $\theta_{2}\left(y_{1}\right)=t_{1}+t_{2}$ such that $z_{1}+t_{1}=\theta_{1}\left(x_{2}\right)$ and $z_{2}+t_{2}=\theta_{2}\left(y_{2}\right)$. Since $\theta_{1}(M)$ is an order-ideal in $Q$, we get $z_{1}, z_{2} \in \theta_{1}(M)$, and similarly $t_{1}, t_{2} \in \theta_{2}(N)$. So we can write $z_{i}=\theta_{1}\left(v_{i}\right)$ and $t_{i}=\theta_{2}\left(w_{i}\right)$, where $v_{i} \in M$ and $w_{i} \in N$. Since the $\theta_{i}$ are injective we get $x_{1}=v_{1}+v_{2}$ and $y_{1}=w_{1}+w_{2}$. Now $\theta_{1}\left(v_{2}\right) \in \theta_{1}(M) \cap \theta_{2}(N)=\theta_{1}(I)$, so we get $v_{2} \in I$. Similarly we get $w_{1} \in \varphi(I)$ and also $x_{2}=v_{1}+\varphi^{-1}\left(w_{1}\right)$ and $y_{2}=\varphi\left(v_{2}\right)+w_{2}$. We have

$$
\left[\left(x_{1}, y_{1}\right)\right]=\left[\left(v_{1}+v_{2}, w_{1}+w_{2}\right)\right]=\left[\left(v_{1}+\varphi^{-1}\left(w_{1}\right), \varphi\left(v_{2}\right)+w_{2}\right)\right]=\left[\left(x_{2}, y_{2}\right)\right] .
$$

This shows that $\iota$ is injective, as desired. 
We now study the notion of a crowned pushout, that we will need in our main construction.

Let $P$ be a conical monoid. Suppose that $P$ contains order-ideals $I$ and $I^{\prime}$, with $I \cap I^{\prime}=0$, such that there is an isomorphism $\varphi: I \rightarrow I^{\prime}$. We have a diagram

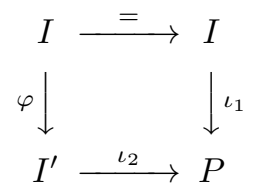

which is not commutative.

The crowned pushout $Q$ of $\left(P, I, I^{\prime}, \varphi\right)$ is by definition the coequalizer of the maps $\iota_{1}: I \rightarrow P$ and $\iota_{2} \circ \varphi: I \rightarrow P$, so that there is a map $f: P \rightarrow Q$ with $f\left(\iota_{1}(x)\right)=f\left(\iota_{2}(\varphi(x))\right)$ for all $x \in I$ and given any other map $g: P \rightarrow Q^{\prime}$ such that $g\left(\iota_{1}(x)\right)=g\left(\iota_{2}(\varphi(x))\right)$ for all $x \in I$, we have that $g$ factors uniquely through $f$.

In the situation of Lemma 4.1, the pushout can be obtained as the crowned pushout of $(M \times N, I \times 0,0 \times \varphi(I), \varphi)$.

Proposition 4.2. Let $P$ be a conical refinement monoid. Suppose that $P$ contains order-ideals $I$ and $I^{\prime}$, with $I \cap I^{\prime}=0$, such that there is an isomorphism $\varphi: I \rightarrow I^{\prime}$. Let $Q$ be the crowned pushout of $\left(P, I, I^{\prime}, \varphi\right)$. Then $Q$ is the monoid $P / \sim$, where $\sim$ is the congruence on $P$ generated by $x+i \sim x+\varphi(i)$ for $i \in I$ and $x \in P$. Moreover $Q$ is a conical refinement monoid, and $Q$ contains an order-ideal $Z$, isomorphic with $I$, such that the projection map $\pi: P \rightarrow Q$ induces an isomorphism $P /\left(I+I^{\prime}\right) \cong Q / Z$.

Proof. It is clear that the canonical projection $\pi: P \rightarrow P / \sim$ is the coequalizer of the maps $\iota_{1}$ and $\iota_{2} \circ \varphi$.

It is straightforward to show that $\sim$ is a refining relation on $P$; that is, if $\alpha \sim$ $x_{1}+x_{2}$, then we can write $\alpha=\alpha_{1}+\alpha_{2}$ with $\alpha_{i} \sim x_{i}$. Since $P$ is a refinement monoid, it follows that $Q$ is a refinement monoid too. Clearly $Q$ is conical.

Note that $I+I^{\prime} \cong I \times I^{\prime}$, because $P$ is a conical refinement monoid. By Lemma 4.1. the pushout $Z$ of $I \leftarrow I \rightarrow I^{\prime}$ is obtained as $I \times I^{\prime} / \sim^{\prime}$, where $\sim^{\prime}$ is the restriction of the congruence $\sim$ to $I \times I^{\prime} \cong I+I^{\prime}$. It follows easily that $Z$ is an order-ideal of $Q:=P / \sim$. By Lemma 4.1, the map $I \rightarrow Z$ is an isomorphism.

Since the projection map $P \rightarrow P /\left(I+I^{\prime}\right)$ clearly equalizes the maps $\iota_{1}: I \rightarrow P$ and $\iota_{2} \circ \varphi: I \rightarrow P$, we get a unique factorization $P \rightarrow Q \rightarrow P /\left(I+I^{\prime}\right)$. We get therefore a factorization of the identity map

$$
P /\left(I+I^{\prime}\right) \rightarrow Q / Z \rightarrow P /\left(I+I^{\prime}\right) .
$$

Since the map $P /\left(I+I^{\prime}\right) \rightarrow Q / Z$ is surjective we get that the map

$$
P /\left(I+I^{\prime}\right) \longrightarrow Q / Z
$$

is an isomorphism.

Lemma 4.3 ([14, Lemma 6.3]). Let I be an order-ideal of a primitive monoid $M$. Then $M / I$ is also a primitive monoid and the canonical map $\pi: M \rightarrow M / I$ induces a $\triangleleft$-isomorphism from $\mathbb{P}(M) \backslash \mathbb{P}(I)$ onto $\mathbb{P}(M / I)$.

The following result determines the structure of the crowned pushout for a primitive monoid.

Lemma 4.4. Let $P$ be a primitive monoid. Suppose that $P$ contains order-ideals $I$ and $I^{\prime}$, with $I \cap I^{\prime}=0$, such that there is an isomorphism $\varphi: I \rightarrow I^{\prime}$. Let $Q$ be 
the crowned pushout of $\left(P, I, I^{\prime}, \varphi\right)$. Then $Q$ is a primitive monoid with $\mathbb{P}(Q)=$ $\mathbb{P}(P) \backslash \mathbb{P}\left(I^{\prime}\right)$, with the order relation induced by the order relation in $\mathbb{P}(P)$ and the additional relations $p<q$ whenever $p \in \mathbb{P}(I), q \in \mathbb{P}(P) \backslash\left(\mathbb{P}(I) \sqcup \mathbb{P}\left(I^{\prime}\right)\right)$ and $\varphi(p)<q$ in $\mathbb{P}(P)$. Moreover, for $p \in \mathbb{P}(Q)$, we have that $p$ is free in $Q$ if and only if $p$ is free in $P$.

Proof. By Proposition 4.2, $Q$ contains an order-ideal $Z$, isomorphic with $I$, such that $Q / Z \cong P /\left(I+I^{\prime}\right)$. Moreover $Q$ is a conical refinement monoid. To show that $Q$ is antisymmetric, we define for $p \in \mathbb{P}(P) \backslash \mathbb{P}\left(I^{\prime}\right)$, some maps $\phi_{p}^{Q}: Q \rightarrow \mathbb{Z}^{\infty}=$ $\mathbb{Z}^{+} \cup\{\infty\}$, by

$$
\phi_{p}^{Q}([x])= \begin{cases}\phi_{p}^{P}(x) & \text { if } p \in \mathbb{P}(P) \backslash\left(\mathbb{P}(I) \sqcup \mathbb{P}\left(I^{\prime}\right)\right), \\ \phi_{p}^{P}(x)+\phi_{\varphi(p)}^{P}(x) & \text { if } p \in \mathbb{P}(I) .\end{cases}
$$

Here $\phi_{p}^{P}(x)=\sup \{n \in \mathbb{Z}: n p \leq x\}$ are the functions associated to the primitive monoid $P$; see (1.1). It is easy to show from the definition of the relation $\sim$ that the maps $\phi_{p}^{Q}$ are well defined, and that they define an injective monoid homomorphism $\phi^{Q}: Q \rightarrow \prod_{p \in \mathbb{P}(P) \backslash \mathbb{P}\left(I^{\prime}\right)} \mathbb{Z}^{\infty}$, from which it follows that $Q$ is antisymmetric. Thus we get that $Q$ is a primitive monoid.

By Lemma 4.3, we have

$$
\mathbb{P}(Q) \backslash \mathbb{P}(Z)=\mathbb{P}(Q / Z)=\mathbb{P}\left(P /\left(I+I^{\prime}\right)\right)=\mathbb{P}(P) \backslash\left(\mathbb{P}(I) \sqcup \mathbb{P}\left(I^{\prime}\right)\right),
$$

and so $\mathbb{P}(Q)=\mathbb{P}(Z) \sqcup\left(\mathbb{P}(P) \backslash\left(P(I) \sqcup \mathbb{P}\left(I^{\prime}\right)\right)\right)=\mathbb{P}(P) \backslash \mathbb{P}\left(I^{\prime}\right)$, and clearly the function $\phi^{Q}$ defined above is the canonical function associated to the primitive monoid $Q$. The rest of the proof follows from this.

Now we will describe a construction with von Neumann regular rings which produces the effect of the crowned pushout of Proposition4.2 at the level of monoids of projectives. Moreover we have a great deal of control on the ring so produced.

We will need the theory of Morita equivalence for rings with local units. We refer the reader to [4] for basic information on this theory (see also [1] and 22]). Recall that $R$ is a ring with local units if every finite subset of $R$ is contained in a ring of the form $e R e$, where $e=e^{2} \in R$. Every von Neumann regular ring is a ring with local units, by [4, Example 1]. When working with modules over rings with local units, one assumes that these modules are unitary. For instance, if $M$ is a right module over $R$, this means that $M=M R$. Equivalently for every finite number of elements $x_{1}, \ldots, x_{n}$ in $M$ there is an idempotent $e$ in $R$ such that $x_{i} e=x_{i}$ for all $i$.

Let $I$ and $I^{\prime}$ be two rings with local units. We say that $I$ and $I^{\prime}$ are Morita equivalent in case there are (unitary) bimodules ${ }_{I} N_{I^{\prime}}$ and ${ }_{I^{\prime}} M_{I}$ and bimodule isomorphisms $N \otimes_{I^{\prime}} M \rightarrow I$ and $M \otimes_{I} N \rightarrow I^{\prime}$ satisfying the following associativity laws: $(n m) n^{\prime}=n\left(m n^{\prime}\right)$ and $(m n) m^{\prime}=m\left(n m^{\prime}\right)$, for all $n, n^{\prime} \in N$ and $m, m^{\prime} \in M$.

Proposition 4.5. Let $R$ be a (not necessarily unital) von Neumann regular ring with ideals $I$ and $I^{\prime}$ such that $I \cap I^{\prime}=0$, and suppose that $I$ and $I^{\prime}$ are Morita equivalent. Then there is a von Neumann regular ring $U$ with an ideal $J$ such that the following holds:

(1) There exists an injective ring homomorphism $\alpha: R \rightarrow U$ such that $\alpha(I) \subseteq J$ and $\alpha\left(I^{\prime}\right) \subseteq J$. If $R$ is unital, then $U$ is unital and $\alpha$ is a unital map.

(2) The map $\mathcal{V}(\alpha): \mathcal{V}(R) \rightarrow \mathcal{V}(U)$ restricts to an isomorphism from $\mathcal{V}(I)$ onto $\mathcal{V}(J)$, and it also restricts to an isomorphism from $\mathcal{V}\left(I^{\prime}\right)$ onto $\mathcal{V}(J)$. 
(3) Let $\varphi: \mathcal{V}(I) \rightarrow \mathcal{V}\left(I^{\prime}\right) \subseteq \mathcal{V}(R)$ be the isomorphism defined by

$$
\varphi:=\left(\mathcal{V}(\alpha)_{\mid \mathcal{V}\left(I^{\prime}\right)}\right)^{-1} \circ\left(\mathcal{V}(\alpha)_{\mid \mathcal{V}(I)}\right) .
$$

Then $\mathcal{V}(\alpha): \mathcal{V}(R) \rightarrow \mathcal{V}(U)$ is the coequalizer of the following (noncommutative) diagram:

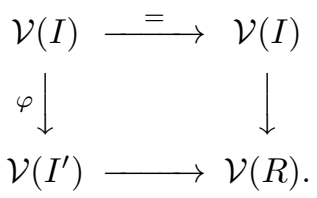

Proof. (1) Let ${ }_{I} N_{I^{\prime}}$ and ${ }_{I^{\prime}} M_{I}$ be (unitary) bimodules implementing a Morita equivalence between $I$ and $I^{\prime}$, so that there are bimodule isomorphisms $N \otimes_{I^{\prime}} M \rightarrow I$ and $M \otimes_{I} N \rightarrow I^{\prime}$ satisfying the associativity laws $(n m) n^{\prime}=n\left(m n^{\prime}\right)$ and $(m n) m^{\prime}=$ $m\left(n m^{\prime}\right)$, for all $n, n^{\prime} \in N$ and $m, m^{\prime} \in M$.

The following diagram is a pullback:

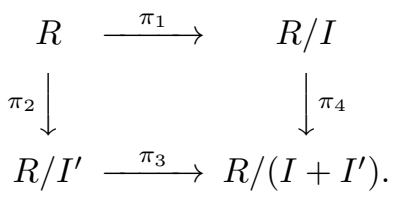

Consider the ring $U$ whose elements are matrices

$$
X=\left(\begin{array}{cc}
r_{1}+I^{\prime} & n \\
m & r_{2}+I
\end{array}\right)
$$

such that $\pi_{3}\left(r_{1}+I^{\prime}\right)=\pi_{4}\left(r_{2}+I\right)$, where $r_{1}+I^{\prime} \in R / I^{\prime}$ and $r_{2}+I \in R / I$, and $n \in N$ and $m \in M$. Since the diagram above is a pullback, we see that for a matrix $X$ as above, there is a unique $r \in R$ such that $r_{1}+I^{\prime}=\pi_{2}(r)$ and $r_{2}+I=\pi_{1}(r)$, which gives another way of describing the elements of $U$. Set $J=\left(\begin{array}{cc}I & N \\ M & I^{\prime}\end{array}\right)$, which is an ideal of $U$. The map $\alpha: R \rightarrow U$ is defined by $\alpha(r)=\operatorname{diag}\left(\pi_{2}(r), \pi_{1}(r)\right) \in U$. Clearly $U$ and $\alpha$ are unital if so is $R$.

It is easy to check that $J$ has local units. Since $J$ is Morita equivalent to $I$ (and to $I^{\prime}$ ) and has local units, it follows from [4, Proposition 3.1] that $J$ is von Neumann regular. Since $J$ and $U / J \cong R / I^{\prime} \times R / I$ are von Neumann regular, it follows from [24, Lemma 1.3] that $U$ is von Neumann regular.

(2) Observe that $\alpha(I)=\left(\begin{array}{ll}I & 0 \\ 0 & 0\end{array}\right)$ and similarly $\alpha\left(I^{\prime}\right)=\left(\begin{array}{cc}0 & 0 \\ 0 & I^{\prime}\end{array}\right)$. Since $J$ is Morita equivalent to both $I$ and $I^{\prime}$, we conclude that the maps $\mathcal{V}(\alpha)_{\mid \mathcal{V}(I)}$ and $\mathcal{V}(\alpha)_{\mid \mathcal{V}\left(I^{\prime}\right)}$ are both isomorphisms onto $\mathcal{V}(J)$; see [26, Corollary 5.6].

(3) The coequalizer of the maps in the diagram (4.1) is constructed in Proposition 4.2 as $\mathcal{V}(R) / \sim$, where $\sim$ is the congruence on $\mathcal{V}(R)$ generated by $x+i \sim x+\varphi(i)$ for every $x \in \mathcal{V}(R)$ and $i \in \mathcal{V}(I)$. Clearly there exists a unique monoid homomorphism $\rho: \mathcal{V}(R) / \sim \longrightarrow \mathcal{V}(U)$ such that $\rho([x])=\mathcal{V}(\alpha)(x)$ for every $x \in \mathcal{V}(R)$, where $[x]$ denotes the equivalence class of $x \in \mathcal{V}(R)$ in $\mathcal{V}(R) / \sim$. We have to show that the map $\rho$ is an isomorphism.

Since our construction is compatible with the passage to matrices of arbitrary size, we can restrict our considerations to idempotents in $R$ and $U$. The following observation will be crucial for our proof. 
Given a finite number of elements $x_{1}, \ldots, x_{n}, y_{1}, \ldots, y_{m} \in J$ and given any "diagonal" idempotent $e=\left(\begin{array}{cc}e_{1}+I^{\prime} & 0 \\ 0 & e_{2}+I\end{array}\right) \in U$ such that $e x_{i}=x_{i}$ for all $i$ and $y_{j} e=y_{j}$ for all $j$, there exist idempotents $g_{1} \in I$ and $g_{2} \in I^{\prime}$ such that $g:=\left(\begin{array}{cc}g_{1} & 0 \\ 0 & g_{2}\end{array}\right) \in J$ satisfies $g \leq e$ and $g x_{i}=x_{i}$ for all $i$ and $y_{j} g=y_{j}$ for all $j$.

This observation is easily proved by taking into account that the sets of idempotents in $I$ and $I^{\prime}$ are directed and the facts that $N=I N=N I^{\prime}$ and $M=I^{\prime} M=$ $M I$.

Let us show first that $\rho$ is surjective. Let $e$ be an idempotent in $U$. Since $U / J \cong R /\left(I+I^{\prime}\right)$, there is an idempotent $f \in R$ such that $\alpha(f)+J=e+J$. From [24, Proposition 2.19] we get the orthogonal decompositions $e=e_{1}+e_{2}$ and $\alpha(f)=f_{1}+f_{2}$ such that $e_{1} \sim f_{1}$ and $e_{2}, f_{2} \in J$. Since $\mathcal{V}(\alpha)_{\mid \mathcal{V}(I)}$ is an isomorphism from $\mathcal{V}(I)$ onto $\mathcal{V}(J)$, we see that it is enough to show that $\mathcal{V}\left(f_{1}\right) \in \mathcal{V}(\alpha)(\mathcal{V}(R))$. (Here $\mathcal{V}\left(f_{1}\right)$ denotes the class of $f_{1}$ in $\mathcal{V}(U)$.) By the above observation we can find idempotents $g_{1} \in I$ and $g_{2} \in I^{\prime}$ such that $g:=\left(\begin{array}{cc}g_{1} & 0 \\ 0 & g_{2}\end{array}\right) \in J$ satisfies $g \leq \alpha(f)$ and $f_{2} \leq g$. Now we have an orthogonal decomposition

$$
f_{1}=(\alpha(f)-g)+\left(g-f_{2}\right)
$$

with $\alpha(f)-g \in \alpha(R)$ and $g-f_{2} \in J$. Consequently, both $\mathcal{V}(\alpha(f)-g)$ and $\mathcal{V}\left(g-f_{2}\right)$ belong to the image of $\mathcal{V}(\alpha)$, and we get

$\mathcal{V}(e)=\mathcal{V}\left(e_{1}\right)+\mathcal{V}\left(e_{2}\right)=\mathcal{V}\left(f_{1}\right)+\mathcal{V}\left(e_{2}\right)=\mathcal{V}(\alpha(f)-g)+\mathcal{V}\left(g-f_{2}\right)+\mathcal{V}\left(e_{2}\right) \in \mathcal{V}(\alpha)(\mathcal{V}(R))$

as required.

Finally we prove the injectivity of $\rho$. Assume that $e$ and $f$ are idempotents in $R$ such that $\mathcal{V}(\alpha(e))=\mathcal{V}(\alpha(f))$ in $\mathcal{V}(U)$. There exist $x \in \alpha(e) U \alpha(f)$ and $y \in \alpha(f) U \alpha(e)$ such that $x y=\alpha(e)$ and $y x=\alpha(f)$. Write $x=\left(\begin{array}{cc}r_{1}+I^{\prime} & n_{1} \\ m_{1} & r_{1}+I\end{array}\right)$ and $y=\left(\begin{array}{cc}r_{2}+I^{\prime} & n_{2} \\ m_{2} & r_{2}+I\end{array}\right)$, where $r_{1}, r_{2} \in R$ and $n_{i} \in N$ and $m_{i} \in M$ for $i=$ 1,2. Applying the observation above to $x_{1}=\left(\begin{array}{cc}0 & n_{1} \\ m_{1} & 0\end{array}\right)$ and $y_{1}=\left(\begin{array}{cc}0 & n_{2} \\ m_{2} & 0\end{array}\right)$, and to the idempotent $\alpha(e)$, we get idempotents $g_{1} \in I$ and $g_{2} \in I^{\prime}$ such that $g:=\left(\begin{array}{cc}g_{1} & 0 \\ 0 & g_{2}\end{array}\right) \in J$ satisfies $g \leq e$ and moreover $g x_{1}=x_{1}$ and $y_{1} g=y_{1}$. But now $\alpha(e)=g+(\alpha(e)-g)$ and $\alpha(f)=y g x+y(\alpha(e)-g) x$, with $y g x \sim g$ in $J$ and

$$
\begin{aligned}
y(\alpha(e)-g) x= & \left(\begin{array}{cc}
\left(r_{2}+I^{\prime}\right)\left(e-g_{1}+I^{\prime}\right) & 0 \\
0 & \left(r_{2}+I\right)\left(e-g_{2}+I\right)
\end{array}\right) \\
& \cdot\left(\begin{array}{cc}
\left(e-g_{1}+I^{\prime}\right)\left(r_{1}+I^{\prime}\right) & 0 \\
0 & \left(e-g_{2}+I\right)\left(r_{1}+I\right)
\end{array}\right)
\end{aligned}
$$

is equivalent to $\alpha(e)-g$ in $\alpha(R)$. Note that also $y g x \in J$. Thus it remains to show that if $\operatorname{diag}\left(e_{1}, f_{1}\right) \sim \operatorname{diag}\left(e_{2}, f_{2}\right)$ in $J$, then $\left[\mathcal{V}\left(e_{1}\right)+\mathcal{V}\left(f_{1}\right)\right]=\left[\mathcal{V}\left(e_{2}\right)+\mathcal{V}\left(f_{2}\right)\right]$ in $\mathcal{V}(R) / \sim$. Using refinement (in $J$ ) we get the orthogonal decompositions $e_{1}=$ $h_{1}+h_{2}$ and $f_{1}=h_{1}^{\prime}+h_{2}^{\prime} \operatorname{such}$ that $\operatorname{diag}\left(h_{1}, h_{1}^{\prime}\right) \sim \operatorname{diag}\left(e_{2}, 0\right)$ and $\operatorname{diag}\left(h_{2}, h_{2}^{\prime}\right) \sim$ 
$\operatorname{diag}\left(0, f_{2}\right)$. So we have in $\mathcal{V}(R) / \sim$,

$$
\begin{aligned}
{\left[\mathcal{V}\left(e_{1}\right)+\mathcal{V}\left(f_{1}\right)\right] } & =\left[\mathcal{V}\left(h_{1}\right)+\mathcal{V}\left(h_{2}\right)+\mathcal{V}\left(h_{1}^{\prime}\right)+\mathcal{V}\left(h_{2}^{\prime}\right)\right] \\
& =\left[\mathcal{V}\left(h_{1}\right)+\varphi\left(\mathcal{V}\left(h_{2}\right)\right)+\mathcal{V}\left(h_{1}^{\prime}\right)+\mathcal{V}\left(h_{2}^{\prime}\right)\right] \\
& =\left[\mathcal{V}\left(h_{1}\right)+\varphi^{-1}\left(\mathcal{V}\left(h_{1}^{\prime}\right)\right)+\varphi\left(\mathcal{V}\left(h_{2}\right)\right)+\mathcal{V}\left(h_{2}^{\prime}\right)\right] \\
& =\left[\mathcal{V}\left(e_{2}\right)+\mathcal{V}\left(f_{2}\right)\right] .
\end{aligned}
$$

This completes the proof of the proposition.

As an easy example to illustrate Proposition 4.5. we consider the case $R=I \oplus I^{\prime}$ with $I \cong I^{\prime} \cong K$ as rings. Then the ring $U$ produced in the proof of 4.5 is just the ring $U=M_{2}(K)$ of $2 \times 2$ matrices over $K$, and the map $\alpha: R \rightarrow U$ is just the embedding along the diagonal.

We will also need a description of certain pullbacks of primitive monoids

Proposition 4.6. Let $M_{1}$ and $M_{2}$ be primitive monoids. Let $N_{i}$ be an order-ideal in $M_{i}$ such that $M_{1} / N_{1} \cong M_{2} / N_{2} \cong S$ and let $P$ be the pullback of $M_{1} \longrightarrow S \longleftarrow M_{2}$. Assume that $p \triangleleft q$ for all $p \in \mathbb{P}\left(N_{i}\right)$ and all $q \in \mathbb{P}\left(M_{i}\right) \backslash \mathbb{P}\left(N_{i}\right), i=1,2$. Then the pullback $P$ is also a primitive monoid and $P$ has an order-ideal $N \cong N_{1} \times N_{2}$ such that $P / N \cong S$. Moreover one has $\mathbb{P}(P)=\mathbb{P}(S) \sqcup \mathbb{P}\left(N_{1}\right) \sqcup \mathbb{P}\left(N_{2}\right)$, with the order relation $\triangleleft$ given by the relations $\triangleleft$ of each monoid $S, N_{1}$ and $N_{2}$, and the further relations $p \triangleleft q$ for all $p \in \mathbb{P}\left(N_{1}\right) \sqcup \mathbb{P}\left(N_{2}\right)$ and $q \in \mathbb{P}(S)$.

Proof. By Lemma 4.3, $S$ is a primitive monoid and $\mathbb{P}(S)$ is $\triangleleft$-isomorphic to $\mathbb{P}\left(M_{i}\right) \backslash \mathbb{P}\left(N_{i}\right)$ for $i=1,2$. Let $Q$ be the primitive monoid determined by the set of primes $\mathbb{P}(Q)=\mathbb{P}(S) \sqcup \mathbb{P}\left(N_{1}\right) \sqcup \mathbb{P}\left(N_{2}\right)$, with the relations inherited by each monoid and $p \triangleleft q$ for all $p \in \mathbb{P}\left(N_{1}\right) \sqcup \mathbb{P}\left(N_{2}\right)$ and $q \in \mathbb{P}(S)$. The result that $P \cong Q$ follows easily from the existence of the commutative diagram

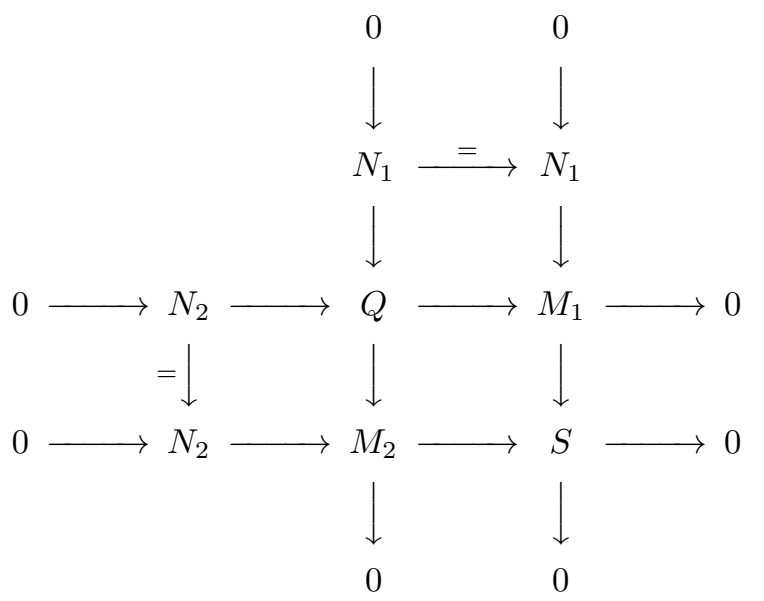

where here a short exact sequence such as $0 \rightarrow N \rightarrow M \rightarrow T \rightarrow 0$ means that $N$ is an order-ideal of $M$ such that $M / N \cong T$.

\section{The BUILDING BLOCKS}

In this section we recall some notation and results from [7] and [8] that we will need in the proof of our main result. For the sake of clarity, we will give a direct proof (modulo some basic results in [7] and 8]) of the result which we need in the present paper. 
In the following, $K$ will denote a field and $E=\left(E^{0}, E^{1}, r, s\right)$ a finite quiver (oriented graph). Here $s(e)$ is the source vertex of the arrow $e$, and $r(e)$ is the range vertex of $e$. A path in $E$ is either an ordered sequence of arrows $\alpha=e_{1} \cdots e_{n}$ with $r\left(e_{t}\right)=s\left(e_{t+1}\right)$ for $1 \leqslant t<n$, or a path of length 0 corresponding to a vertex $v \in E^{0}$. The paths $v$ are called trivial paths, and we have $r(v)=s(v)=v$. A nontrivial path $\alpha=e_{1} \cdots e_{n}$ has length $n$ and we define $s(\alpha)=s\left(e_{1}\right)$ and $r(\alpha)=r\left(e_{n}\right)$. We will denote the length of a path $\alpha$ by $|\alpha|$, the set of all paths of length $n$ by $E^{n}$, and the set of all paths by $E^{*}$.

We define a relation $\geq$ on $E^{0}$ by setting $v \geq w$ if there is a path $\mu \in E^{*}$ with $s(\mu)=v$ and $r(\mu)=w$. A subset $H$ of $E^{0}$ is called hereditary if $v \geq w$ and $v \in H$ imply $w \in H$. A set is saturated if every vertex which feeds into $H$ and only into $H$ is again in $H$, that is, if $s^{-1}(v) \neq \emptyset$ and $r\left(s^{-1}(v)\right) \subseteq H$ imply $v \in H$.

In this paper we will only need a special case of the general construction. For any positive integer $r \geq 0$, we consider the graph $E_{r}$ consisting of $r+1$ vertices $v_{0}, \ldots, v_{r}$ and for each $1 \leq i \leq r$, two arrows $a_{i}, b_{i}$ such that $s\left(a_{i}\right)=r\left(a_{i}\right)=s\left(b_{i}\right)=v_{i}$ and $r\left(b_{i}\right)=v_{i-1}$. A picture of $E_{3}$ is shown in Figure 3 . Observe that $\left(E_{r}^{0}, \leq\right)$ is a chain of length $r$. The monoid $M\left(E_{r}\right)$ of [13] agrees with the the monoid $M\left(E_{r}^{0}\right)$ associated with the poset $E_{r}^{0}$.

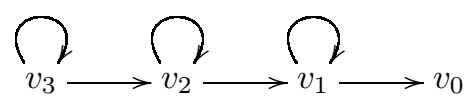

Figure 3 . The quiver $E_{3}$.

Observe that there is a unique maximal chain of hereditary saturated subsets of $E_{r}^{0}$ given by

$$
\mathbf{H}_{r}:\left\{v_{0}\right\} \subset\left\{v_{0}, v_{1}\right\} \subset \cdots \subset E_{r}^{0} .
$$

Let us recall the construction from [7] of the regular algebra $Q_{K}(E)$ of a quiver $E$. Unfortunately the arrows are taken in the present paper in the reverse sense as in [7. So we recall the basic features of the regular algebra $Q_{K}(E)$ in terms of the notation used here. The algebra $Q_{K}(E)$ fits into the following commutative diagram of injective algebra morphisms:

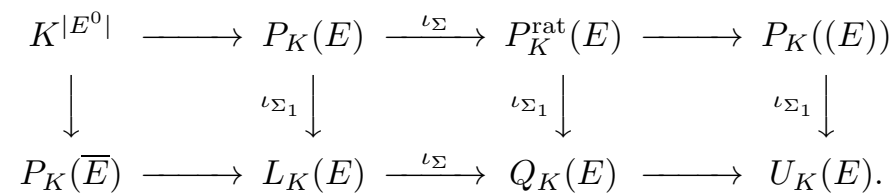

Here $P_{K}(E)$ is the path $K$-algebra of $E, \bar{E}$ denotes the inverse quiver of $E$, that is, the quiver obtained by changing the orientation of all the arrows in $E, P_{K}((E))$ is the algebra of formal power series on $E$, and $P_{K}^{\text {rat }}(E)$ is the algebra of rational series, which is by definition the division closure of $P_{K}(E)$ in $P((E)$ ) (which agrees with the rational closure [7, Observation 1.18]). The maps $\iota_{\Sigma}$ and $\iota_{\Sigma_{1}}$ indicate universal localizations with respect to the sets $\Sigma$ and $\Sigma_{1}$, respectively. Here $\Sigma$ is the set of all square matrices over $P_{K}(E)$ that are sent to invertible matrices by the augmentation map $\epsilon: P_{K}(E) \rightarrow K^{\left|E^{0}\right|}$. By [7, Theorem 1.20], the algebra $P_{K}^{\text {rat }}(E)$ coincides with the universal localization $P_{K}(E) \Sigma^{-1}$. For $v \in E^{0}$ with $s^{-1}(v) \neq \emptyset$, write $s^{-1}(v)=\left\{e_{1}^{v}, \ldots, e_{n_{v}}^{v}\right\}$. The set $\Sigma_{1}=\left\{\mu_{v} \mid v \in E^{0}, s^{-1}(v) \neq \emptyset\right\}$ is the set of 
morphisms between finitely generated projective left $P_{K}(E)$-modules defined by

$$
\begin{aligned}
\mu_{v}: P_{K}(E) v & \longrightarrow \bigoplus_{i=1}^{n_{v}} P_{K}(E) r\left(e_{i}^{v}\right) \\
r & \longmapsto\left(r e_{1}^{v}, \ldots, r e_{n_{v}}^{v}\right)
\end{aligned}
$$

for any $v \in E^{0}$ such that $s^{-1}(v) \neq \emptyset$. By a slight abuse of notation, we also use $\mu_{v}$ to denote the corresponding maps between finitely generated projective left $P_{K}^{\mathrm{rat}}(E)$-modules and $P_{K}((E))$-modules, respectively.

The following relations hold in $Q_{K}(E)$ :

(1) $v v^{\prime}=\delta_{v, v^{\prime}} v$ for all $v, v^{\prime} \in E^{0}$.

(2) $s(e) e=e r(e)=e$ for all $e \in E^{1}$.

(3) $r(e) \bar{e}=\bar{e} s(e)=\bar{e}$ for all $e \in E^{1}$.

(4) $\bar{e} e^{\prime}=\delta_{e, e^{\prime}} r(e)$ for all $e, e^{\prime} \in E^{1}$.

(5) $v=\sum_{\left\{e \in E^{1} \mid s(e)=v\right\}} e \bar{e}$ for every $v \in E^{0}$ that emits edges.

The Leavitt path algebra $L_{K}(E)=P_{K}(E) \Sigma_{1}^{-1}$ is the algebra generated by $\{v \mid$ $\left.v \in E^{0}\right\} \cup\left\{e, \bar{e} \mid e \in E^{1}\right\}$ subject to the relations (1)-(5) above. By 7, Theorem 4.2], the algebra $Q_{K}(E)$ is a von Neumann regular hereditary ring and $Q_{K}(E)=$ $P_{K}(E)\left(\Sigma \cup \Sigma_{1}\right)^{-1}$. Here the set $\Sigma$ can be clearly replaced with the set of all square matrices of the form $I_{n}+B$ with $B \in M_{n}\left(P_{K}(E)\right)$ satisfying $\epsilon(B)=0$, for all $n \geq 1$, since the map $\epsilon: P_{K}(E) \rightarrow K^{\left|E^{0}\right|}$ is a split surjection. The graph monoid $M(E)$ of $E$ is defined as the quotient monoid of $F=F_{E}$, the free abelian monoid with basis $E^{0}$, modulo the congruence generated by the relations

$$
v=\sum_{\left\{e \in E^{1} \mid s(e)=v\right\}} r(e)
$$

for every vertex $v \in E^{0}$ such that $s^{-1}(v) \neq \emptyset$. It was proved in [13, Theorem 3.5] that the natural map $M(E) \rightarrow \mathcal{V}\left(L_{K}(E)\right)$ is an isomorphism, and in 7, Theorem 4.2 that the map $\mathcal{V}\left(L_{K}(E)\right) \rightarrow \mathcal{V}\left(Q_{K}(E)\right)$ induced by the inclusion $L_{K}(E) \rightarrow$ $Q_{K}(E)$ is also an isomorphism.

The structure of the lattice of ideals of $Q_{K}(E)$ can be neatly computed from the graph. Let $H$ be a hereditary saturated subset of $E^{0}$. Define the graph $E / H$ by $(E / H)^{0}=E^{0} \backslash H$ and $(E / H)^{1}=\left\{e \in E^{1}: r(e) \notin H\right\}$, with the functions $r$ and $s$ inherited from $E$. We also define $E_{H}$ as the restriction of the graph $E$ to $H$, that is, $\left(E_{H}\right)^{0}=H$ and $\left(E_{H}\right)^{1}=\left\{e \in E^{1}: s(e) \in H\right\}$. For $Y \subseteq E^{0}$ set $p_{Y}=\sum_{v \in Y} v$.

Proposition 5.1. (a) The ideals of $Q_{K}(E)$ are in one-to-one correspondence with the order-ideals of $M(E)$ and consequently with the hereditary and saturated subsets of $E$.

(b) If $H$ is a hereditary saturated subset of $E$, then $Q_{K}(E) / I_{K}(H) \cong Q_{K}(E / H)$, where $I_{K}(H)$ is the ideal of $Q_{K}(E)$ generated by the idempotent $p_{H}=\sum_{v \in H} v$.

(c) Let $H$ be a hereditary subset of $E^{0}$. Then the following properties hold:

(1) $P_{K}\left(E_{H}\right)=p_{H} P_{K}(E)=p_{H} P_{K}(E) p_{H}$,

(2) $P_{K}\left(\left(E_{H}\right)\right)=p_{H} P_{K}((E))=p_{H} P_{K}((E)) p_{H}$,

(3) $P_{K}^{r a t}\left(E_{H}\right)=p_{H} P_{K}^{r a t}(E)=p_{H} P_{K}^{r a t}(E) p_{H}$,

(4) $Q_{K}\left(E_{H}\right) \cong p_{H} Q_{K}(E) p_{H}$.

Proof. (a) By [7, Theorem 4.2] we have a monoid isomorphism $\mathcal{V}\left(Q_{K}(E)\right) \cong M(E)$. Since $Q_{K}(E)$ is von Neumann regular, we have a lattice isomorphism $\mathcal{L}\left(Q_{K}(E)\right) \cong$ $\mathcal{L}(M(E))$; see Proposition [1.1, Now by [13, Proposition 5.2] there is a lattice 
isomorphism $\mathcal{L}(M(E)) \cong \mathcal{H}$, where $\mathcal{H}$ is the lattice of hereditary saturated subsets of $E^{0}$.

(b), (c) See 8 .

We are ready to define the basic building blocks to apply the diagram constructions.

Definition 5.2. Let $\mathbf{K}_{r}: K_{0} \subseteq K_{1} \subseteq \cdots \subseteq K_{r}$ be a chain of fields. Let $E_{r}$ be the quiver defined above and let $\mathbf{H}_{r}: H_{0}=\left\{v_{0}\right\} \subset H_{1}=\left\{v_{0}, v_{1}\right\} \subset \cdots \subset H_{r}=E_{r}^{0}$ be the unique maximal chain of hereditary saturated subsets of $E_{r}^{0}$. Build rings $R_{i}$, $i=0,1, \ldots, r$, inductively as follows:

(1) $R_{0}=Q_{K_{r}}\left(E_{H_{0}}\right) \cong K_{r}$.

(2) $R_{i}=Q_{K_{r-i}}\left(E_{H_{i}}\right)+Q_{K_{r-i}}\left(E_{H_{i}}\right) p_{H_{i-1}} R_{i-1} p_{H_{i-1}} Q_{K_{r-i}}\left(E_{H_{i}}\right)$ for $1 \leq i \leq r$.

Each $R_{i}$ is a unital $K_{r-i}$-algebra with unit $p_{H_{i}}$ and we have $Q_{K_{r-i}}\left(E_{H_{i}}\right) \subseteq R_{i} \subseteq$ $Q_{K_{r}}\left(E_{H_{i}}\right)=p_{H_{i}} Q_{K_{r}}\left(E_{r}\right) p_{H_{i}} \subseteq Q_{K_{r}}\left(E_{r}\right)$. Put $Q_{\mathbf{K}_{r}}\left(E_{r} ; \mathbf{H}_{r}\right)=R_{r}$.

Theorem 5.3. With the above notation, we have that $Q_{\mathbf{K}_{r}}\left(E_{r} ; \mathbf{H}_{r}\right)$ is a von Neumann regular ring and the natural map $M\left(E_{r}\right) \rightarrow \mathcal{V}\left(Q_{\mathbf{K}_{r}}\left(E_{r} ; \mathbf{H}_{r}\right)\right)$ is an isomorphism.

Proof. We proceed by induction on $r$. For $r=0$, we have $Q_{\mathbf{K}_{0}}\left(E_{0} ; \mathbf{H}_{0}\right) \cong K_{0}$, so the result trivially holds.

Assume that $r>0$ and that the result holds for $r-1$. Let $I_{r-1}$ be the ideal of $Q_{\mathbf{K}_{r}}\left(E_{r} ; \mathbf{H}_{r}\right)$ generated by $p_{H_{r-1}}=\sum_{i=0}^{r-1} v_{i}$. By Proposition 5.1(b), we have

$$
Q_{\mathbf{K}_{r}}\left(E_{r} ; \mathbf{H}_{r}\right) / I_{r-1} \cong Q_{K_{0}}\left(E_{r} / H_{r-1}\right) \cong K_{0}(z),
$$

the rational function field in one variable over the field $K_{0}$. Thus $Q_{\mathbf{K}_{r}}\left(E_{r} ; \mathbf{H}_{r}\right) / I_{r-1}$ is von Neumann regular and $\mathcal{V}\left(Q_{\mathbf{K}_{r}}\left(E_{r} ; \mathbf{H}_{r}\right) / I_{r-1}\right) \cong \mathbb{Z}^{+}$.

On the other hand we have $([8])$

$$
p_{H_{r-1}} Q_{\mathbf{K}_{r}}\left(E_{r} ; \mathbf{H}_{r}\right) p_{H_{r-1}} \cong Q_{\mathbf{K}^{r-1}}\left(E_{H_{r-1}} ; \mathbf{H}_{r-1}\right),
$$

where

$$
\mathbf{K}^{r-1}: K_{1} \subseteq K_{2} \subseteq \cdots \subseteq K_{r}
$$

and

$$
\mathbf{H}_{r-1}: H_{0} \subset H_{1} \subset \cdots \subset H_{r-1} .
$$

Thus, by the induction hypothesis, $p_{H_{r-1}} Q_{\mathbf{K}_{r}}\left(E_{r} ; \mathbf{H}_{r}\right) p_{H_{r-1}}$ is von Neumann regular and the natural map $M\left(E_{r-1}\right) \rightarrow \mathcal{V}\left(p_{H_{r-1}} Q_{\mathbf{K}_{r}}\left(E_{r} ; \mathbf{H}_{r}\right) p_{H_{r-1}}\right)$ is an isomorphism. Now we want to check that $I_{r-1}$ has local units. Note that

$$
I_{r-1}=\left(Q_{K_{0}}\left(E_{r}\right) p_{H_{r-1}}\right)\left(p_{H_{r-1}} R_{r-1} p_{H_{r-1}}\right)\left(p_{H_{r-1}} Q_{K_{0}}\left(E_{r}\right)\right) .
$$

Given $x \in I_{r-1}$, we can write

$x=\sum_{i=1}^{n} a_{i} z_{i} b_{i}$, with $a_{i} \in Q_{K_{0}}\left(E_{r}\right) p_{H_{r-1}}, z_{i} \in p_{H_{r-1}} R_{r-1} p_{H_{r-1}}, b_{i} \in p_{H_{r-1}} Q_{K_{0}}\left(E_{r}\right)$.

Since $Q_{K_{0}}\left(E_{r}\right) p_{H_{r-1}} Q_{K_{0}}\left(E_{r}\right)$ is von Neumann regular ([7, Theorem 4.2]), it has local units (see, e.g., [4, Example 1]), so there is an idempotent $e \in$ $Q_{K_{0}}\left(E_{r}\right) p_{H_{r-1}} Q_{K_{0}}\left(E_{r}\right) \subseteq I$ such that $e a_{i}=a_{i}$ and $b_{i}=b_{i} e$ for all $i$. It follows that $e x=x=x e$, as desired.

Since $I_{r-1}$ has local units and $I_{r-1}$ is Morita equivalent to the regular ring $p_{H_{r-1}} Q_{\mathbf{K}_{r}}\left(E_{r} ; \mathbf{H}_{r}\right) p_{H_{r-1}}$, it follows from [4, Proposition 3.1] that $I_{r-1}$ is von Neumann regular. By using this and (5.1), it follows from [24, Lemma 1.3] that 
$Q_{\mathbf{K}_{r}}\left(E_{r} ; \mathbf{H}_{r}\right)$ is von Neumann regular. Moreover since it is an extension of two strongly separative regular rings, we get that $Q_{\mathbf{K}_{r}}\left(E_{r} ; \mathbf{H}_{r}\right)$ is also strongly separative ([11, Theorem 5.2]).

Observe that by the Morita invariance of the functor $\mathcal{V}(-)$ (see 26, Corollary 5.6]) it follows that the natural map $M\left(E_{r-1}\right) \rightarrow \mathcal{V}\left(I_{r-1}\right)$ is an isomorphism.

We now want to compute the monoid $\mathcal{V}\left(Q_{\mathbf{K}_{r}}\left(E_{r} ; \mathbf{H}_{r}\right)\right)$. Write $Q:=Q_{\mathbf{K}_{r}}\left(E_{r}\right.$; $\left.\mathbf{H}_{r}\right)$. We have algebra embeddings $Q_{K_{0}}\left(E_{r}\right) \rightarrow Q \rightarrow Q_{K_{r}}\left(E_{r}\right)$ which induce monoid homomorphisms

$$
M\left(E_{r}\right) \cong \mathcal{V}\left(Q_{K_{0}}\left(E_{r}\right)\right) \rightarrow \mathcal{V}(Q) \rightarrow \mathcal{V}\left(Q_{K_{r}}\left(E_{r}\right)\right) \cong M\left(E_{r}\right) .
$$

Observe that the composition above is the identity, so it follows that the map

$$
\mathcal{V}\left(Q_{K_{0}}\left(E_{r}\right)\right) \rightarrow \mathcal{V}(Q)
$$

is injective. In order to see that it is surjective we have to prove that every idempotent in $Q$ is equivalent to a direct sum of basic idempotents in $E_{r}^{0}$. Take an idempotent $e$ in $Q$. If $e \in I_{r-1}$, then the result follows from the isomorphism $M\left(E_{r-1}\right) \cong \mathcal{V}\left(I_{r-1}\right)$. If $e \notin I_{r-1}$, then $\left[e+I_{r-1}\right]=[1]$ by (5.1), and thus we get $e \oplus f_{1} \sim 1 \oplus f_{2}$ for some idempotents $f_{1}, f_{2} \in I_{r-1}$. Now since $1 \oplus f \sim 1$ for every idempotent $f \in I_{r-1}$, we get

$$
e \oplus f_{1} \sim 1 \oplus f_{2} \sim 1 \sim 1 \oplus f_{1},
$$

so that $e \oplus f_{1} \sim 1 \oplus f_{1}$. Since $f_{1} \leq 1$ and $Q$ is strongly separative we get that $e \sim 1$, as desired.

We conclude that the natural map $M\left(E_{r}\right) \rightarrow \mathcal{V}(Q)$ is an isomorphism, as wanted.

\section{THE PROOF OF THE MAIN THEOREM}

This section is devoted to the proof of our main result, Theorem 2.3. Let $M$ be a finitely generated primitive monoid with all primes free, and let $\mathbb{P}=\mathbb{P}(M)$ be the poset of primes of $M$. The proof of Theorem 2.3 is naturally divided into two big steps, as follows:

Step 1. For a maximal element $p$ of $\mathbb{P}$, the poset $\mathbb{P} \downarrow p=\{q \in \mathbb{P}: q \leq p\}$ has $p$ as a greatest element, and we build in Proposition 6.1 a poset $\mathbb{F}(p)$, with greatest element $p$, such that, for each $t \in \mathbb{F}(p)$, the interval $[t, p]$ is a chain, together with an order-preserving surjective map $\Psi_{p}: \mathbb{F}(p) \rightarrow \mathbb{P} \downarrow p$. The poset $\mathbb{F}(p)$ is built up from the different maximal chains $S$ of $\mathbb{P} \downarrow p$, and Proposition 4.6 tells us that $M(\mathbb{P} \downarrow p)=M(\mathbb{P}) \mid p$ is built up from the corresponding monoids $M(S)$ by a finite sequence of pullback diagrams. Finally to each maximal chain $S$ we associate a basic building block $Q(S)$ of the sort considered in Section 5, in such a way that, using Theorem 3.2, we are able to prove that the same sequence of pullback diagrams applied now to the $K$-algebras $Q(S)$ leads us to the algebra $Q_{K}(\mathbb{F}(p))$ of the poset $\mathbb{F}(p)$ (Definition 2.1), so that Theorem 2.3 is proved for the posets $\mathbb{F}(p)$. This is achieved in Theorem 6.3. This step uses Sections 2, 3] and 5, as well as Proposition 4.6 .

Step 2. Let $\Psi_{p}: \mathbb{F}(p) \rightarrow \mathbb{P} \downarrow p$ be the map of posets described in Step 1. This map can be extended to a surjective monoid homomorphism $\Psi_{p}: M(\mathbb{F}(p)) \rightarrow M(\mathbb{P}) \mid p$. We show in Proposition 6.5 that $M(\mathbb{P}) \mid p$ is obtained from $M(\mathbb{F}(p))$ by a finite sequence of crowned pushouts of the form considered in Lemma 4.4. Moreover 
$M(\mathbb{P})$ is also obtained from $\prod_{p \in \operatorname{Max}(\mathbb{P})} M(\mathbb{P}) \mid p$ by a sequence of crowned pushouts as in Lemma 4.4. Now Proposition 4.5 gives a way to construct, from a given von Neumann regular ring $R$ such that $\mathcal{V}(R) \cong M$, and from a suitable crowned pushout $M^{\prime}$ of $M$, a von Neumann regular ring $U$ such that $\mathcal{V}(U) \cong M^{\prime}$. Since we already know that Theorem 2.3 holds for the posets $\mathbb{F}(p)$ (Step 1), the result in the general case follows by an inductive argument from Proposition 4.5. once we are able to identify (modulo Morita equivalence) the algebra $U$ for $R=Q_{K}(M)$, with the corresponding algebra $Q_{K}\left(M^{\prime}\right)$, where $M$ is one of the monoids appearing in the sequence of crowned pushouts leading from the different monoids $M(\mathbb{F}(p))$, for $p \in \operatorname{Max}(\mathbb{P})$, to $M(\mathbb{P})$, and $M^{\prime}$ is obtained from $M$ by a crowned pushout construction as shown in the proof of Proposition 6.5. This identification is done in Theorem 6.6. Step 2 uses Sections 2 and 4

Let $M$ be a finitely generated primitive monoid such that all the primes of $M$ are free. The height of a prime $p \in \mathbb{P}(M)$ is the length $r$ of a maximal chain of primes $p_{0}<p_{1}<\cdots<p_{r}=p$. The height of $M$ is the maximum of the heights of its prime elements. Similar definitions apply to (elements of) a finite poset.

We first state a purely order-theoretic result.

Proposition 6.1. Let $\mathbb{P}(p)$ be a finite poset with a greatest element $p$, and assume that the height of $p$ is $r$. Let $\mathcal{S}^{0}(p)$ be the set of all maximal chains in $\mathbb{P}(p)$ of the form $p_{0}<p_{1}<\cdots<p_{s}=p$, and let $\Psi_{0}: \bigsqcup_{S \in \mathcal{S}^{0}(p)} S \rightarrow \mathbb{P}(p)$ be the natural surjective identification map. Then there is a sequence of sets $\mathcal{S}^{1}(p), \mathcal{S}^{2}(p), \ldots, \mathcal{S}^{r}(p)$ such that:

(i) $\mathcal{S}^{i}(p)$ consists of partially ordered sets $T$ with maximum element $p$ such that for each $t \in T$ the set $[t, p]=\{x \in T: t \leq x\}$ is a chain. Moreover $T$ contains a chain $p_{i}<\cdots<p_{r}=p$ and every element in $T$ not in this chain is below $p_{i}$, that is, $T=\left\{x \in T: x<p_{i}\right\} \sqcup\left\{p_{i}, \ldots, p_{r}\right\}$.

(ii) For each $i$ there is a surjective identification order-preserving map

$$
\Psi_{i}: \bigsqcup_{S \in \mathcal{S}^{i}(p)} S \rightarrow \mathbb{P}(p) .
$$

(iii) $\mathcal{S}^{r}(p)$ is a singleton $\{\mathbb{F}(p)\}$, so there is a surjective identification order-preserving map

$$
\Psi_{r}: \mathbb{F}(p) \rightarrow \mathbb{P}(p) .
$$

Proof. In order to make the process clear, we will start by constructing $\mathcal{S}^{1}(p)$. Let us define an equivalence relation on the set $\mathcal{S}^{0}(p)$ by setting $S \sim S^{\prime}$ iff either $S=S^{\prime}$ or $S \neq S^{\prime}$ and $p_{i}=p_{i}^{\prime}$ for $i=1, \ldots, r$. In the latter case, we are assuming that both $S$ and $S^{\prime}$ have maximal length $r$. Each chain in $\mathcal{S}^{0}(p)$ of length $<r$ forms a singleton class with respect to $\sim$. Now $\mathcal{S}^{1}(p)$ contains all the chains $S$ in a singleton class and one new partially ordered set for each equivalence class with more than one element. We construct this element for a given class $\left\{S_{1}, \ldots, S_{\alpha}\right\}$. Let $p_{0,1}, \ldots, p_{0, \alpha}$ be the minimal elements in each one of the chains $S_{1}, \ldots, S_{\alpha}$, and let $p_{1}<\cdots<p_{r}$ be the common part of the chains. Then the set $T$ corresponding to this class has elements $p_{0,1}, \ldots, p_{0, \alpha}, p_{1}, \ldots, p_{r}$ and the order relation determined by $p_{0, i}<p_{1}<\cdots<p_{r}$ for all $i$. There is an obvious surjective identification order-preserving map

$$
\Psi_{1}: \bigsqcup_{T \in \mathcal{S}^{1}(p)} T \rightarrow \mathbb{P}(p) .
$$


Now the construction of $\mathcal{S}^{i}(p)$ from $\mathcal{S}^{i-1}(p)$ is similar to the first step. By the induction hypothesis, the set $\mathcal{S}^{i-1}(p)$ consists of partially ordered sets $T$ with maximum element $p$ such that for each $t \in T$ the set $[t, p]=\{x \in T: t \leq x\}$ is a chain. Moreover $T$ contains a chain $p_{i-1}<p_{i}<\cdots<p_{r}=p$ (with $p_{\ell-1} \in$ $\mathrm{L}\left(\mathbb{P}(p), p_{\ell}\right)$ for $\left.\ell=i, \ldots, r\right)$ and $T=\left\{x \in T: x<p_{i-1}\right\} \sqcup\left\{p_{i-1}, \ldots, p_{r}\right\}$. We also have surjective identification maps

$$
\Psi_{i-1}: \bigsqcup_{T \in \mathcal{S}^{i-1}(p)} T \rightarrow \mathbb{P}(p) .
$$

Now define an equivalence relation on $\mathcal{S}^{i-1}(p)$ by $T \sim T^{\prime}$ iff $p_{j}=p_{j}^{\prime}$ for $j=$ $i, i+1, \ldots, r$, where $T$ contains the chain $p_{i-1}<p_{i}<\cdots<p_{r}=p$ and $T^{\prime}$ contains the chain $p_{i-1}^{\prime}<p_{i}^{\prime}<\cdots<p_{r}^{\prime}=p$, with $p_{\ell-1} \in \mathrm{L}\left(\mathbb{P}(p), p_{\ell}\right)$ and $p_{\ell-1}^{\prime} \in \mathrm{L}\left(\mathbb{P}(p), p_{\ell}^{\prime}\right)$ for $\ell=i, \ldots, r$.

The set $\mathcal{S}^{i}(p)$ contains all the posets $T$ in a singleton class of $S^{i-1}(p)$ and one new partially ordered set for each equivalence class with more than one element. For a given class $\left\{T_{1}, \ldots, T_{k}\right\}$, take

$$
W=\left\{p_{i}, p_{i+1}, \ldots, p_{r}\right\} \sqcup \bigsqcup_{j=1}^{k}\left\{t \in T_{j}: t<p_{i}\right\},
$$

with the obvious order relation. The restriction of $\Psi_{i-1}$ to $\bigsqcup_{j=1}^{k} T_{j}$ factors through $W$, so we obtain a surjective order-preserving map $\Psi_{i}: \bigsqcup_{W \in \mathcal{S}^{i}(p)} W \rightarrow \mathbb{P}(p)$. For $i=r$, we get a unique poset $\mathbb{F}(p)$.

Note that the map $\Psi_{r}: \mathbb{F}(p) \rightarrow \mathbb{P}(p)$ is an isomorphism if and only if $\mathbb{P}(p)$ satisfies that $[t, p]$ is a chain for every $t \in \mathbb{P}(p)$. The first step of the proof of Theorem 2.3 consists in showing it in the particular case where the poset $\mathbb{P}(M)$ has a maximum element $p$ and $[t, p]$ is a chain for every $t \in \mathbb{P}(M)$ (Theorem 6.3).

For any positive integer $r \geq 1$, we consider the graph $E_{r}$ consisting of $r+1$ vertices $v_{0}, \ldots, v_{r}$ and for each $1 \leq i \leq r$, two arrows $a_{i}, b_{i}$ such that $s\left(a_{i}\right)=r\left(a_{i}\right)=$ $s\left(b_{i}\right)=v_{i}$ and $r\left(b_{i}\right)=v_{i-1}$. Let $M_{r}$ be the graph monoid of $E_{r}$. Then $\mathbb{P}\left(M_{r}\right)$ is a chain of length $r$, that is, it consists of $r+1$ primes $p_{0}, p_{1}, \ldots, p_{r}$ corresponding to $v_{0}, v_{1}, \ldots, v_{r}$, respectively, such that

$$
p_{0}<p_{1}<\cdots<p_{r} \text {. }
$$

Now we consider a construction as in Section [5 with respect to the following data. Let $K\left(t_{1}, t_{2}, \ldots\right)$ be an infinite purely transcendental extension of a field $K$. Select positive integers $1 \leq k_{1} \leq k_{2} \leq \cdots \leq k_{r}$ and set

$$
\mathbf{K}_{r}: K\left(t_{k_{r}}, t_{k_{r}+1}, \ldots\right) \subseteq K\left(t_{k_{r-1}}, t_{k_{r-1}+1}, \ldots\right) \subseteq \cdots \subseteq K\left(t_{1}, t_{2}, \ldots\right)=L .
$$

We also consider the unique maximal chain of hereditary saturated subsets of $E_{r}^{0}$,

$$
\mathbf{H}_{r}:\left\{v_{0}\right\} \subset\left\{v_{0}, v_{1}\right\} \subset \cdots \subset E_{r}^{0} .
$$

By Theorem 5.3, the ring $Q_{\mathbf{K}_{r}}\left(E_{r} ; \mathbf{H}_{r}\right)$ is von Neumann regular and $\mathcal{V}\left(Q_{\mathbf{K}_{r}}\left(E_{r} ; \mathbf{H}_{r}\right)\right)$ $=M_{r}$. First we want to compare this construction with the construction in Definition 2.1. For this we consider a slight variation, denoted by $Q\left(M_{r}, \sigma\left(k_{1}, \ldots, k_{r}\right)\right)$, of the definition in 2.1, where the $K$-endomorphisms $\sigma^{p_{i}}: L \rightarrow L$ used in (2.8) are replaced with the ones defined by the rule

$$
\sigma^{p_{i}}\left(t_{j}\right)=t_{j+k_{i}-k_{i-1}} .
$$


Observe that $Q\left(M_{r}, \sigma\left(k_{1}, \ldots, k_{r}\right)\right)$ has the same essential properties as $Q_{K}\left(M_{r}\right)$ (cf. Section 2).

Proposition 6.2. With the above notation we have an isomorphism of $K$-algebras

$$
\gamma: Q\left(M_{r}, \sigma\left(k_{1}, k_{2}, \ldots, k_{r}\right)\right) \longrightarrow Q_{\mathbf{K}_{r}}\left(E_{r} ; \mathbf{H}_{r}\right)
$$

such that $\gamma\left(e\left(p_{i}\right)\right)=v_{i}$ for $i \geq 0, \gamma\left(\alpha_{p_{i}, p_{i-1}}\right)=a_{i}, \gamma\left(\beta_{p_{i}, p_{i-1}}\right)=b_{i}$ and $\gamma\left(e\left(p_{i}\right) t_{j}\right)=$ $v_{i} t_{j+k_{i}-1}$ for $i \geq 1$.

Proof. Write $\alpha_{i}=\alpha_{p_{i}, p_{i-1}}$ and $\beta_{i}=\beta_{p_{i}, p_{i-1}}$. Observe that $Q\left(M_{r}, \sigma\left(k_{1}, k_{2}, \ldots, k_{r}\right)\right)$ $=\mathcal{A}\left(\Sigma \cup \Sigma_{1}\right)^{-1}$ (see Definition 2.1).

Define a $K$-algebra map $\widetilde{\gamma}: \mathcal{A} \rightarrow Q_{\mathbf{K}_{r}}\left(E_{r} ; \mathbf{H}_{r}\right)$ by the rules:

$$
\begin{aligned}
\widetilde{\gamma}\left(e\left(p_{i}\right)\right) & =v_{i}, \quad \widetilde{\gamma}\left(\alpha_{i}\right)=a_{i}, \quad \widetilde{\gamma}\left(\beta_{i}\right)=b_{i}, \\
\widetilde{\gamma}\left(e\left(p_{i}, p_{i-1}\right)\right) & =b_{i} \bar{b}_{i}, \quad \text { and } \quad \widetilde{\gamma}\left(e\left(p_{i}\right) t_{j}\right)=v_{i} t_{j+k_{i}-1} .
\end{aligned}
$$

Since relations (2.3), (2.8) and (2.10) are respected by $\widetilde{\gamma}$, the map $\widetilde{\gamma}$ is well defined. Clearly the map $\widetilde{\gamma}$ is $\left(\Sigma \cup \Sigma_{1}\right)$-inverting, so that we have a well-defined $K$-algebra morphism

$$
\gamma: Q\left(M_{r}, \sigma\left(k_{1}, \ldots, k_{r}\right)\right) \rightarrow Q_{\mathbf{K}_{r}}\left(E_{r} ; \mathbf{H}_{r}\right) .
$$

In order to show that $\gamma$ is surjective, it is enough to show, in view of Definition 5.2, that $Q_{K_{r-i}}\left(E_{H_{i}}\right) \subseteq \operatorname{Im}(\gamma)$. For this, we will define a map $\tau_{i}: Q_{K_{r-i}}\left(E_{H_{i}}\right) \rightarrow$ $Q\left(M_{r}, \sigma\left(k_{1}, k_{2}, \ldots, k_{r}\right)\right)$ such that $\gamma \tau_{i}=\operatorname{id}_{Q_{K_{r-i}}\left(E_{H_{i}}\right)}$. Recall that the algebra $Q_{K_{r-i}}\left(E_{H_{i}}\right)$ is the universal localization of the path algebra $P_{K_{r-i}}\left(E_{H_{i}}\right)$ with respect to $\Sigma\left(\epsilon_{i}\right) \cup \Xi_{i}$, where $\epsilon_{i}: P_{K_{r-i}}\left(E_{H_{i}}\right) \rightarrow K_{r-i}^{i+1}$ is the augmentation map, and $\Xi_{i}=\left\{\mu_{v_{j}}: j=1, \ldots, i\right\}$, with

$$
\begin{aligned}
\mu_{v_{j}}: P_{K_{r-i}}\left(E_{H_{i}}\right) v_{j} & \longrightarrow P_{K_{r-i}}\left(E_{H_{i}}\right) v_{j} \oplus P_{K_{r-i}}\left(E_{H_{i}}\right) v_{j-1}, \\
r & \longmapsto\left(r a_{j}, r b_{j}\right) .
\end{aligned}
$$

Define a map $\tilde{\tau}: P_{K_{r-i}}\left(E_{H_{i}}\right) \rightarrow\left(\sum_{j=0}^{i} e\left(p_{i}\right)\right) Q\left(M_{r}, \sigma\left(k_{1}, \ldots, k_{r}\right)\right)\left(\sum_{j=0}^{i} e\left(p_{i}\right)\right)$ by the rule

$$
\widetilde{\tau}\left(v_{j}\right)=e\left(p_{j}\right), \quad \widetilde{\tau}\left(a_{j}\right)=\alpha_{j}, \quad \widetilde{\tau}\left(b_{j}\right)=\beta_{j}, \quad \widetilde{\tau}\left(v_{j} t_{\ell}\right)=e\left(p_{j}\right) t_{\ell-k_{j}+1} .
$$

The map is clearly $\Xi_{i}$-inverting. We have to show that $\widetilde{\tau}$ is $\Sigma\left(\epsilon_{i}\right)$-inverting. Set $P=P_{K_{r-i}}\left(E_{H_{i}}\right)$, and take any matrix $A \in M_{n}(P)$ such that $\epsilon_{i}(A)=0$. Observe that we can write

$$
A=A_{i}+B_{i}+A_{i-1}+B_{i-1}+\cdots+A_{1}+B_{1},
$$

with $A_{j} \in v_{j} M_{n}(P) v_{j}$ and $B_{j} \in v_{j} M_{n}(P)\left(v_{j-1}+\cdots+v_{0}\right)$, and with $\epsilon_{i}\left(A_{j}\right)=$ $\epsilon_{i}\left(B_{\ell}\right)=0$ for all $j, \ell$. Since

$$
\left(I_{n}-A\right)^{-1}=\left(I_{n}-A_{i}\right)^{-1}\left(I_{n}-B_{i}\right)^{-1}\left(I_{n}-A_{i-1}\right)^{-1} \cdots\left(I_{n}-A_{1}\right)^{-1}\left(I_{n}-B_{1}\right)^{-1}
$$

in any ring in which all the terms on the RHS are invertible, it suffices to show that the terms $I_{n}-\widetilde{\tau}\left(A_{j}\right)$ and $I_{n}-\widetilde{\tau}\left(B_{j}\right)$ are invertible in $Q\left(M_{r}, \sigma\left(k_{1}, \ldots, k_{r}\right)\right)$ for all $j \leq i$. Since $B_{j}^{2}=0$, the matrices $I_{n}-B_{j}$ are obviously invertible. On the other hand, since, for $j \geq 1$, we have $v_{j} P v_{j} \cong K_{r-i}[z]$, we get that

$$
\operatorname{det}\left(I_{n}-\widetilde{\tau}\left(A_{j}\right)\right)=1-f_{j}\left(\alpha_{j}\right),
$$


where $f_{j} \in L[z]$ satisfies $f_{j}(0)=0$. It follows that $e\left(p_{j}\right)-f_{j}\left(\alpha_{j}\right) \in \Sigma\left(p_{j}\right) \subseteq \Sigma$ (see (2.6) and (2.7)) and thus $I_{n}-\widetilde{\tau}\left(A_{j}\right)$ is invertible in $Q\left(M_{r}, \sigma\left(k_{1}, \ldots, k_{r}\right)\right)$ for all $j$. It follows that there is a unique extension of $\widetilde{\tau}$ to a $K_{r-i}$-algebra map

$$
\tau_{i}: Q_{K_{r-i}}\left(E_{H_{i}}\right) \longrightarrow\left(\sum_{j=0}^{i} e\left(p_{i}\right)\right) Q\left(M_{r}, \sigma\left(k_{1}, \ldots, k_{r}\right)\right)\left(\sum_{j=0}^{i} e\left(p_{i}\right)\right) .
$$

Since the composition $\gamma \tau_{i}$ is the identity on $P_{K_{r-i}}\left(E_{H_{i}}\right)$, it must be the identity also on $Q_{K_{r-i}}\left(E_{H_{i}}\right)$.

We have shown that the map $\gamma$ is surjective. The injectivity of $\gamma$ follows easily from Lemma 2.13 .

We are now ready to show the following particular case of our main result:

Theorem 6.3. Let $M$ be a finitely generated primitive monoid such that all its primes are free. Assume that $\mathbb{P}(M)$ has a greatest element $p$ and that $[t, p]$ is a chain for every $t \in \mathbb{P}(M)$. Then $Q_{K}(M)=Q_{K}(\mathbb{P}(M))$ is a von Neumann regular ring and the natural monoid homomorphism

$$
\psi: M \rightarrow \mathcal{V}\left(Q_{K}(M)\right)
$$

is an isomorphism.

Proof. Write $n_{p}:=|\mathrm{L}(\mathbb{P}, p)|$ for $p \in \mathbb{P}:=\mathbb{P}(M)$. We will use the notation of Proposition 6.1 For each $S \in \mathcal{S}^{0}(p)$ of the form $S=\left\{p_{0}, p_{1}, \ldots, p_{s}\right\}$, where $p_{0}<$ $p_{1}<\cdots<p_{s}=p$ is a maximal chain in $\mathbb{P}$, we consider the $K$-algebra $Q(S)$ defined by $Q(S)=Q\left(M(S), \sigma\left(k_{1}, \ldots, k_{s}\right)\right)$, where $k_{1}=1$ and $k_{2}=n_{p_{1}}$, and in general $k_{j}=k_{j-1}+n_{p_{j-1}}-1$, for $j=2, \ldots, s$. Indeed we will find it more useful to use simply the notation $Q(S)=Q\left(M(S),\left(1, n_{p_{1}}, \ldots, n_{p_{s-1}}\right)\right)$ to denote this algebra. Recall that in $Q(S)$ the $K$-endomorphisms $\sigma^{p}: L \rightarrow L$ used in relation (2.8) are given by

$$
\sigma^{p_{1}}\left(t_{\ell}\right)=t_{\ell}, \quad \sigma^{p_{j}}\left(t_{\ell}\right)=t_{\ell+n_{j-1}-1} \quad(2 \leq j \leq s) .
$$

We are going to give a corresponding definition of $Q(T)$ for every $T \in \mathcal{S}^{i}(p)$. Take any $T \in \mathcal{S}^{i}(p)$. Then $T$ is a subset of $\mathbb{P}$ of the form

$$
T=\left\{p_{i}, p_{i+1}, \ldots, p_{r}\right\} \sqcup\left\{q \in \mathbb{P}: q<p_{i}\right\},
$$

where $p_{i}<p_{i+1}<\cdots<p_{r}=p$ is a chain that cannot be refined. (This uses of course our hypothesis that $[t, p]$ is a chain for every $t$ in $\mathbb{P}$.) Define

$$
Q(T)=Q_{K}\left(M(T),\left(1, n_{p_{i+1}}, \ldots, n_{p_{r-1}}\right)\right),
$$

where the $K$-algebra $Q_{K}\left(M(T),\left(1, n_{p_{i+1}}, \ldots, n_{p_{r-1}}\right)\right)$ is the one defined in 2.1 with the only difference that we use for $\sigma^{p_{i+1}}, \sigma^{p_{i+2}}, \ldots, \sigma^{p_{r}}$ the $K$-endomorphisms of $L$ defined by:

$$
\sigma^{p_{i+1}}\left(t_{\ell}\right)=t_{\ell}, \quad \sigma^{p_{j}}\left(t_{\ell}\right)=t_{\ell+n_{j-1}-1} \quad(i+2 \leq j \leq r) .
$$

All the $\sigma^{q}$ for $q \leq p_{i}$ are the same as in the definition of $Q_{K}(M(T))$, and they agree with the ones used for these primes in the definition of $Q_{K}(\mathbb{P}(M))$, i.e. $\sigma^{q}\left(t_{\ell}\right)=$ $t_{\ell+n_{q}-1}$ when $q \leq p_{i}$.

We are going to prove by induction on $i$ the following statement:

$\left(\mathrm{S}_{i}\right)$ For every $0 \leq i \leq r$ and every $T \in \mathcal{S}^{i}(p)$, the $K$-algebra $Q(T)$ is von Neumann regular and $\mathcal{V}(Q(T)) \cong M(T)$. In particular $Q(T)$ is a strongly separative von Neumann regular ring. 
For $i=0$, this is given by Proposition 6.2. Assume that $r \geq i>0$ and that statement $\left(\mathrm{S}_{i-1}\right)$ holds. We are going to show $\left(\mathrm{S}_{i}\right)$. Let $\left\{T_{1}, \ldots, T_{k}\right\}$ be a nontrivial class in $\mathcal{S}^{i-1}(p)$, and let

$$
q_{j}<p_{i}<p_{i+1}<\cdots<p_{r}=p
$$

be the chain corresponding to $T_{j}$, for $j=1, \ldots, k$. In order to simplify notation, we will denote $n_{\ell}=n_{p_{\ell}}$ for $i \leq \ell \leq r$, and similarly $\alpha_{i j}:=\alpha_{p_{i}, q_{j}}, \beta_{i j}:=\beta_{p_{i}, q_{j}}$, $\alpha_{\ell}:=\alpha_{p_{\ell}, p_{\ell-1}}, \beta_{\ell}:=\beta_{p_{\ell}, p_{\ell-1}}$ for $i+1 \leq \ell \leq r$. Let $T \in \mathcal{S}^{i}(p)$ be given by

$$
T=\left\{p_{i}, p_{i+1}, \ldots, p_{r}\right\} \sqcup \bigsqcup_{j=1}^{k}\left\{t \in T_{j}: t<p_{i}\right\} .
$$

Observe that $\mathrm{L}\left(T, p_{i}\right)=\mathrm{L}\left(\mathbb{P}, p_{i}\right)=\left\{q_{1}, \ldots, q_{k}\right\}$, so that $k=n_{i}$, and $\mathrm{L}\left(T, p_{u}\right)=$ $\left\{p_{u-1}\right\}$ for $r \geq u \geq i+1$. By Proposition 4.6 we see that $M(T)$ is the pullback of the family of natural maps $M\left(T_{j}\right) \rightarrow M(\Lambda)$, where $\Lambda$ is the chain $\left\{p_{i}, \ldots, p_{r}\right\}$. (In categorical terms, the family of maps $\left\{M(T) \rightarrow M\left(T_{j}\right): j=1, \ldots, k\right\}$, defined by sending all $q \in T$, with $q \leq q_{\ell}$ for $\ell \neq j$, to 0 , are the limit of the family of maps $\left\{M\left(T_{j}\right) \rightarrow M(\Lambda): j=1, \ldots, k\right\}$ in the category of monoids.)

Set $Q:=Q\left(\Lambda,\left(n_{i}+1, n_{i+1}, \ldots, n_{r-1}\right)\right)$. Let $I_{j}$ be the ideal of $Q\left(T_{j}\right)$ generated by $e\left(q_{j}\right)$. Then there is a natural isomorphism $\tilde{\pi}_{j}: Q\left(T_{j}\right) / I_{j} \rightarrow Q$ which is essentially the identity on generators involving $p_{i+1}, \ldots, p_{r}$ and which, in level $i$, acts as follows:

$$
\begin{gathered}
\tilde{\pi}_{j}\left(e\left(p_{i}\right) t_{\ell}\right)= \begin{cases}e\left(p_{i}\right) t_{\ell} & \text { if } \ell<j, \\
e\left(p_{i}\right) t_{\ell+1} & \text { if } \ell \geq j,\end{cases} \\
\tilde{\pi}_{j}\left(\alpha_{i j}\right)=e\left(p_{i}\right) t_{j} .
\end{gathered}
$$

Let $\pi_{j}: Q\left(T_{j}\right) \longrightarrow Q$ be the map defined by the composition of the canonical projection $Q\left(T_{j}\right) \rightarrow Q\left(T_{j}\right) / I_{j}$ and the isomorphism $\tilde{\pi}_{j}$. Then the $\pi_{j}$ are surjective maps with kernel $I_{j}$. Now, for $1 \leq j \leq k$, consider the $K$-algebra morphism $\rho_{j}: Q(T) \rightarrow Q\left(T_{j}\right)$ which annihilates all $e\left(q_{\ell}\right)$ with $\ell \neq j$, is defined as the identity in levels $i+1, \ldots, r$ and also below $q_{j}$, and on level $i$ is defined as follows:

$$
\begin{gathered}
\rho_{j}\left(\alpha_{i \ell}\right)= \begin{cases}e\left(p_{i}\right) t_{\sigma_{j}(\ell)} & \text { if } \ell \neq j, \\
\alpha_{i j} & \text { if } \ell=j,\end{cases} \\
\rho_{j}\left(\beta_{i \ell}\right)= \begin{cases}0 & \text { if } \ell \neq j, \\
\beta_{i j} & \text { if } \ell=j,\end{cases} \\
\rho_{j}\left(e\left(p_{i}\right) t_{u}\right)=e\left(p_{i}\right) t_{u+k-1} .
\end{gathered}
$$

It is easily seen that these assignments give a well-defined morphism $\rho_{j}$; that is, all relations between generators in Definition 2.1 are preserved and the images by $\rho_{j}$ of maps in $\Sigma \cup \Sigma_{1}$ are invertible in $Q\left(T_{j}\right)$. The homomorphism $\rho_{j}$ is clearly surjective. We have $\pi_{j} \circ \rho_{j}=\pi_{j^{\prime}} \circ \rho_{j^{\prime}}$ for all $j, j^{\prime}$, and so we get a canonical map $\rho: Q(T) \rightarrow P$, where $P$ denotes the limit (pullback) of the maps $\pi_{j}: Q\left(T_{j}\right) \rightarrow Q$. We want to see that $\rho$ is an isomorphism. Observe that $P$ has an ideal of the form $I:=I_{1} \times \cdots \times I_{k}$ and that $P / I \cong Q$ canonically. The idea to show that $\rho$ is an isomorphism is to prove, using the results in Section 2, that the same structure holds in $Q(T)$.

Let $G_{j}$ be the ideal of $Q(T)$ generated by $e\left(q_{j}\right)$. Let $A_{j}=T \downarrow q_{j}=\{x \in T: x \leq$ $\left.q_{j}\right\}$, which is a lower subset of $T$. By construction of $T$, we have that $A_{j} \cap A_{\ell}=\emptyset$ for $j \neq \ell$. It follows from (the proof) of Proposition 2.15(2) that the sum $\sum_{j=1}^{k} G_{j}$ 
is a direct sum. Observe that the kernel of the map $\pi_{j} \circ \rho_{j}$ is precisely $\bigoplus_{j=1}^{k} G_{j}$. On the other hand, the kernel of the map $\rho_{j}: Q(T) \rightarrow Q\left(T_{j}\right)$ is precisely the ideal $\bigoplus_{\ell \neq j} G_{\ell}$, and it follows that $\rho_{j}$ induces an isomorphism $G_{j} \cong I_{j}$ for $j=1, \ldots, k$.

Hence we have a commutative diagram with exact rows:

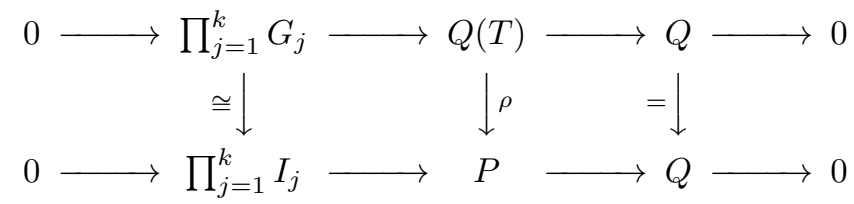

and thus $\rho: Q(T) \rightarrow P$ is an isomorphism. It follows from Theorem 3.2 and $\left(\mathrm{S}_{i-1}\right)$ that $Q(T)$ is a strongly separative von Neumann regular ring.

By the induction hypothesis, $\left(\mathrm{S}_{i-1}\right)$ holds, so that $Q\left(T_{j}\right)$ is a von Neumann regular ring and the natural map $M\left(T_{j}\right) \rightarrow \mathcal{V}\left(Q\left(T_{j}\right)\right)$ is an isomorphism, for $1 \leq$ $j \leq k$. In particular we see that $K_{0}\left(I_{j}\right) \cong \mathbb{Z}$, a generator being $\left[e\left(p_{i}, q_{j}\right)\right]=\left[e\left(q_{j}\right)\right]$.

Now let us show that every idempotent $e$ in $Q(T)$ such that $Q(T) e Q(T) \nsubseteq$ $\prod_{j=1}^{k} G_{j}$ must satisfy $Q(T) e Q(T)=Q(T) e\left(p_{u}\right) Q(T)=Q(T)\left(\sum_{q \leq p_{u}} e(q)\right) Q(T)$ for some $u=i, \ldots, r$. Indeed we have $Q(T) e Q(T)+\prod_{j=1}^{k} G_{j}=Q(T) e\left(p_{u}\right) Q(T)$, for some $u=i, \ldots, r$, and so

$$
e\left(p_{u}\right) \oplus f_{1} \sim m \cdot e \oplus f_{2},
$$

where $f_{2}$ is a finite direct sum of basic idempotents in $G_{j}, j=1, \ldots, k$. Thus $e\left(p_{u}\right) \sim e\left(p_{u}\right) \oplus f_{2}$ and we get

$$
e\left(p_{u}\right) \oplus f_{1} \oplus f_{2} \sim m \cdot e \oplus f_{2} .
$$

Since $Q(T)$ is strongly separative, we get $e\left(p_{u}\right) \oplus f_{1} \sim m \cdot e$ and so $Q(T) e\left(p_{u}\right) Q(T)=$ $Q(T) e Q(T)$.

Therefore, we only need to check the conditions (3.1) of Theorem 3.2 for the idempotents $\sum_{q \leq p_{u}} e(q), i \leq u \leq r$, and indeed, without loss of generality, we can assume that $e=1=\left(1_{Q\left(T_{1}\right)}, \ldots, 1_{Q\left(T_{k}\right)}\right)$. The diagrams are of the form:

$$
K_{1}\left(Q\left(T_{j}\right)\right) \stackrel{\left(\pi_{j}\right)_{*}}{\longrightarrow} K_{1}(Q) \stackrel{\partial_{j}}{\longrightarrow} K_{0}\left(I_{j}\right) \stackrel{0}{\longrightarrow} K_{0}\left(Q\left(T_{j}\right)\right) .
$$

By (6.3) and the definition of the connecting map, we get $\partial_{j}\left(\left[e\left(p_{i}\right) t_{j}\right]\right)=-\left[e\left(p_{i}, q_{j}\right)\right]$. By using (6.2) we see that, for $\ell \neq j$, we have $\left(\pi_{\ell}\right)_{*}\left(\left[e\left(p_{i}\right) t_{j}\right]\right)=\left[e\left(p_{i}\right) t_{j}\right]$ if $j<\ell$ and $\left(\pi_{\ell}\right)_{*}\left(\left[e\left(p_{i}\right) t_{j-1}\right]\right)=\left[e\left(p_{i}\right) t_{j}\right]$ if $j \geq \ell+1$. Thus for $j=1, \ldots, k$ we have

$$
K_{1}(Q)=\left(\pi_{j}\right)_{*}\left(K_{1}\left(Q\left(T_{j}\right)\right)\right)+\left(\bigcap_{\ell \neq j}\left(\pi_{\ell}\right)_{*}\left(K_{1}\left(Q\left(T_{\ell}\right)\right)\right)\right) .
$$

It follows from Theorem 3.2 that the natural map $M(T) \rightarrow \mathcal{V}(Q(T))$ is an isomorphism.

This concludes the proof of Theorem 6.3 .

We turn now to the general case. In this case, the strategy is to analyze how $M(\mathbb{P})$ is obtained from the different monoids $M(\mathbb{F}(p))$, where $p$ ranges over the maximal elements of $\mathbb{P}$.

For a maximal element $p$ of a poset $\mathbb{P}$, the poset $\mathbb{P} \downarrow p$ has $p$ as a greatest element, so we can use the construction in Proposition 6.1 to obtain a poset $\mathbb{F}(p)$ and a surjective order-preserving map $\Psi: \mathbb{F}(p) \rightarrow \mathbb{P} \downarrow p$. The map $\Psi$ preserves chains, that is, if $S$ is a chain in $\mathbb{F}(p)$, then $\Psi$ restricts to a bijection from $S$ to $\Psi(S)$. Moreover it is easy to see that the map $S \rightarrow \Psi(S)$ is a bijection from the set of 
maximal chains of $\mathbb{F}(p)$ onto the set $\mathcal{S}^{0}(p)$ of maximal chains of $\mathbb{P} \downarrow p$. We also recall two fundamental properties of $\mathbb{F}(p)$ :

(1) For every $t \in \mathbb{F}(p)$ the interval $[t, p]$ is a chain (Proposition 5.1(i)).

(2) For $t_{1}, t_{2} \in \mathbb{F}(p)$, if $\Psi\left(\left[t_{1}, p\right]\right)=\Psi\left(\left[t_{2}, p\right]\right)$, then $t_{1}=t_{2}$. (This follows directly from the construction of $\mathbb{F}(p)$.)

Lemma 6.4. For every $q \in \mathbb{F}(p)$, the map $\Psi$ induces a bijection from $\mathrm{L}(\mathbb{F}(p), q)$ onto $\mathrm{L}(\mathbb{P} \downarrow p, \Psi(q))$.

Proof. Write $\mathcal{T}:=\mathcal{T}(\mathbb{P} \downarrow p)$, where $\mathcal{T}(\mathbb{P} \downarrow p)$ is the quiver associated to the poset $\mathbb{P} \downarrow p$; see Definition 2.1. For $q^{\prime} \in \mathbb{P} \downarrow p$ there is a bijection betweeen $\Psi^{-1}\left(q^{\prime}\right)$ and the set of paths in $\mathcal{T}$ from $p$ to $q^{\prime}$. If $\Psi(q)=q^{\prime}$, then an element in $\mathrm{L}(\mathbb{F}(p), q)$ corresponds to an enlargement of the path in $\mathcal{T}$ from $p$ to $q^{\prime}$ corresponding to $q$, by an arrow from $q^{\prime}$ to an element in $\mathrm{L}(\mathbb{P} \downarrow p, q)$. This gives the result.

Since $\Psi\left(q^{\prime}\right)<\Psi(q)$ whenever $q^{\prime}<q$ in $\mathbb{F}(p)$, the map $\Psi: \mathbb{F}(p) \rightarrow \mathbb{P} \downarrow p$ can be extended to a surjective monoid homomorphism, denoted in the same way,

$$
\Psi: M(\mathbb{F}(p)) \longrightarrow M(\mathbb{P} \downarrow p)=M(\mathbb{P}) \mid p .
$$

The depth of an element $q$ in a poset $\mathbb{P}$ is the maximum length of a chain of the form $q=q_{0}<q_{1}<\cdots<q_{t}$. Let us denote the poset of elements of $\mathbb{P}$ of depth $\leq s$ by $\mathbb{P}_{s}$.

Proposition 6.5. Let $M$ be a finitely generated primitive monoid such that all primes of $M$ are free. For a maximal element $p$ of $\mathbb{P}(M)$, consider the monoid homomorphism $\Psi: M(\mathbb{F}(p)) \rightarrow M \mid p$ defined above. Assume that $p$ has height $r$. For $0 \leq i \leq r$, there are primitive monoids $M^{i}(p)$ such that, for $0 \leq i<j \leq r$, there are surjective monoid homomorphisms $\Psi_{j i}: M^{i}(p) \rightarrow M^{j}(p)$ with $\Psi_{j i}=\Psi_{j k} \Psi_{k i}$ when $i<k<j$, such that the following properties are satisfied:

(i) $M^{0}(p)=M(\mathbb{F}(p)), M^{r}(p)=M \mid p$ and $\Psi_{r 0}=\Psi$.

(ii) $\Psi_{i 0}$ induces an isomorphism of posets from $\left(\mathbb{P}\left(M^{0}(p)\right)\right)_{r-i}$ onto $\left(\mathbb{P}\left(M^{i}(p)\right)\right)_{r-i}$.

(iii) $\Psi_{r i}$ induces an isomorphism between $M^{i}(p) \mid q$ and $M \mid \Psi_{r i}(q)$ for $q \in$ $\mathbb{P}\left(M^{i}(p)\right)$ of depth $\geq r-i-1$.

(iv) $\left(M^{i}(p) \mid q_{1}\right) \cap\left(M^{i}(p) \mid q_{2}\right)=0$ for two incomparable elements $q_{1}$ and $q_{2}$ in $\mathbb{P}\left(M^{i}(p)\right)$ of depth $\leq r-i-1$.

The monoid $M^{i+1}(p)$ (respectively, $M$ ) is obtained from the monoid $M^{i}(p)$ (respectively, $\left.\prod_{p \in \operatorname{Max}(\mathbb{P})} M \mid p\right)$ by a finite sequence of crowned pushout diagrams of the form considered in Lemma 4.4 .

Proof. We proceed by induction on $i$.

Observe that order-ideals in $M^{0}(p):=M(\mathbb{F}(p))$ are in bijection with lower subsets of $\mathbb{F}(p)$; see Proposition 1.2. If $q_{1}$ and $q_{2}$ are incomparable elements of $\mathbb{F}(p)$, then $\left(\mathbb{F}(p) \downarrow q_{1}\right) \cap\left(\mathbb{F}(p) \downarrow q_{2}\right)=\emptyset$, because $[t, p]$ is a chain for every $t \in \mathbb{F}(p)$. Hence property (iv) holds for $M^{0}(p)$. Property (ii) holds vacuously, and property (iii) follows from Lemma 6.4.

Assume that $M^{i}(p)$ and $\Psi_{i 0}, \Psi_{r i}$ have been constructed, where $0 \leq i<r-$ 1 , so that properties (i)-(iv) hold. We need to build $M^{i+1}(p)$ so that the map $\Psi_{r i}: M^{i}(p) \rightarrow M \mid p$ factors through $M^{i+1}(p)$. Write $\mathbb{P}^{i}:=\mathbb{P}\left(M^{i}(p)\right)$. Let $q$ be an element in $\mathbb{P}^{i}$ of depth $r-i-2$. Consider the set $\mathrm{L}\left(\mathbb{P}^{i}, q\right)=\left\{q_{1}, \ldots, q_{k}\right\}$ of lower covers of $q$ in $\mathbb{P}^{i}$. Observe that the elements $q_{u}$ have depth exactly $r-i-1$. Indeed, 
$q_{u}$ has depth $\leq r-i-1$, and thus by (ii), $\left[q_{u}, p\right] \cong\left[\Psi_{i 0}^{-1}\left(q_{u}\right), p\right]$, so that $\left[q_{u}, p\right]$ is a chain. It follows that the depth of $q_{u}$ is exactly $r-i-1$.

Since $q_{1}, \ldots, q_{k}$ are mutually incomparable, it follows from (iv) that $\left(M^{i}(p) \mid\right.$ $\left.q_{u}\right) \cap\left(M^{i}(p) \mid q_{v}\right)=0$ for $u \neq v$. Since the depth of $q_{j}$ is $r-i-1$, it follows from (iii) that

$$
\Psi_{r i \mid\left(M^{i}(p) \mid q_{j}\right)}: M^{i}(p)\left|q_{j} \longrightarrow M\right| \Psi_{r i}\left(q_{j}\right)
$$

is an isomorphism, for $j=1, \ldots, k$.

Consider the order-ideals of $M^{i}(p)$ given by:

$$
Z_{u}:=\left(\left(\Psi_{r i}\right)_{\mid\left(M^{i}(p) \mid q_{u}\right)}\right)^{-1}\left(\Psi_{r i}\left(M^{i}(p) \mid q_{1}\right) \cap \Psi_{r i}\left(M^{i}(p) \mid q_{2}\right)\right)
$$

for $u=1,2$. The map

$$
\varphi=\left(\left(\left(\Psi_{r i}\right)_{\mid\left(M^{i}(p) \mid q_{2}\right)}\right)^{-1} \circ\left(\Psi_{r i}\right)_{\mid\left(M^{i}(p) \mid q_{1}\right)}\right)_{\mid Z_{1}}: Z_{1} \longrightarrow Z_{2}
$$

is a monoid isomorphism, and $Z_{u} \subseteq\left(M^{i}(p) \mid q_{u}\right)$ for $u=1,2$. Observe that, by Lemma 6.4, condition (ii), and the fact that $\Psi=\Psi_{r i} \Psi_{i 0}$, it follows that $\Psi_{r i}$ induces a bijection from $\mathrm{L}\left(\mathbb{P}^{i}, q\right)$ onto $\mathrm{L}\left(\mathbb{P} \downarrow p, \Psi_{r i}(q)\right)$. This implies that $Z_{u}$ is strictly contained in $M^{i}(p) \mid q_{u}$ for $u=1,2$.

By (iv) we have $Z_{1} \cap Z_{2}=0$. So we can consider the crowned pushout $M^{\prime}$ of $\left(M^{i}(p), Z_{1}, Z_{2}, \varphi\right)$; see Section 4. By Lemma 4.4. $M^{\prime}$ is a primitive monoid with all primes free, and $\mathbb{P}\left(M^{\prime}\right)=\mathbb{P}^{i} \backslash \mathbb{P}\left(Z_{2}\right)$ with the order structure given by the restriction of the order in $\mathbb{P}^{i}$ and the additional relations, for $p \in \mathbb{P}\left(Z_{1}\right)$ and $q \in \mathbb{P}^{i} \backslash\left(\mathbb{P}\left(Z_{1}\right) \cup \mathbb{P}\left(Z_{2}\right)\right)$, given by $p<q$ if $\varphi(p)<q$ in $\mathbb{P}^{i}$. Moreover, the map $\Psi_{r i}: M^{i}(p) \rightarrow M$ factors as

$$
M^{i}(p) \stackrel{\mu^{\prime}}{\longrightarrow} M^{\prime} \stackrel{\lambda^{\prime}}{\longrightarrow} M,
$$

where the map $\mu^{\prime}: M^{i}(p) \rightarrow M^{\prime}$ can be identified by Lemma 4.4 with the natural identification map sending $p$ and $\varphi(p)$ to $p$ for $p \in \mathbb{P}\left(Z_{1}\right)$, and sending $p$ to $p$ for $p \in \mathbb{P}^{i} \backslash\left(\mathbb{P}\left(Z_{1}\right) \cup \mathbb{P}\left(Z_{2}\right)\right)$. Moreover, the map $\lambda^{\prime}$ induces an isomorphism from $M^{\prime} \mid\left\{q_{1}, q_{2}\right\}$ onto $M \mid\left\{\Psi_{r i}\left(q_{1}\right), \Psi_{r i}\left(q_{2}\right)\right\}$. Proceeding in this way, we obtain a new primitive monoid $M^{\prime \prime}$, such that all primes of $M^{\prime \prime}$ are free, with a factorization of the map $\Psi_{r i}: M^{i}(p) \rightarrow M$ of the form

$$
M^{i}(p) \stackrel{\mu^{\prime \prime}}{\longrightarrow} M^{\prime \prime} \stackrel{\lambda^{\prime \prime}}{\longrightarrow} M,
$$

such that $\lambda^{\prime \prime}$ induces an isomorphism from $M^{\prime \prime} \mid\left\{q_{1}, \ldots, q_{k}\right\}$ onto $M \mid\left\{\Psi_{r i}\left(q_{1}\right), \ldots\right.$, $\left.\Psi_{r i}\left(q_{k}\right)\right\}$, and such that $\mu^{\prime \prime}$ is the identity on all the primes of depth $\leq r-i-1$. Clearly the map $\lambda^{\prime \prime}$ induces an order isomorphism from $\mathbb{P}\left(M^{\prime \prime}\right) \downarrow q$ onto $\mathbb{P} \downarrow \Psi_{r i}(q)$, and thus a monoid isomorphism from $M^{\prime \prime}(p) \mid q$ onto $M \mid \Psi_{r i}(q)$.

Proceeding in this way with all the primes $q$ in $M^{i}(p)$ of depth $r-i-2$, we get a factorization of the map $\Psi_{r i}$ as

$$
M^{i}(p) \stackrel{\Psi_{i+1, i}}{\longrightarrow} M^{i+1}(p) \stackrel{\Psi_{r, i+1}}{\longrightarrow} M \mid p,
$$

with the desired properties. Note that for $i=r-1$, we get an isomorphism $\Psi_{r, r-1}: M^{r-1}(p) \rightarrow M \mid p$.

Finally observe that a similar process can be used to get $M$ from $\prod_{p \in \operatorname{Max}(\mathbb{P})} M \mid p$ by a finite sequence of crowned pushouts.

Let $\mathbb{P}$ be a poset, and let $A$ be a lower subset of $\mathbb{P}$. Put

$$
\partial A=\{p \in \mathbb{P}: \mathrm{L}(\mathbb{P}, p) \cap A \neq \emptyset\} \cup A .
$$


Observe that, as explained at the beginning of this section (Step 2), the next theorem, together with Theorem 6.3 and Proposition 6.5. completes the proof of Theorem 2.3 , because the basic steps used to build $M(\mathbb{P})$ from the monoids $M(\mathbb{F}(p))$, for $p \in \operatorname{Max}(\mathbb{P})$, are of the form described in the theorem (and moreover Theorem 2.3 holds for the monoids $M(\mathbb{F}(p))$ by Theorem 6.3).

Theorem 6.6. Let $M$ be a finitely generated primitive monoid with all primes free. Assume that $\mathbb{P}:=\mathbb{P}(M)$ contains two lower subsets $A, A^{\prime}$ such that $\partial A \cap$ $\partial A^{\prime}=\emptyset$, and assume moreover that there is an order isomorphism $\varphi: A \rightarrow A^{\prime}$. Let $\varphi: M(A) \rightarrow M\left(A^{\prime}\right)$ denote the induced monoid isomorphism, and let $M^{\prime}$ be the crowned pushout of $\left(M, M(A), M\left(A^{\prime}\right), \varphi\right)$.

Suppose that $Q_{K}(M)$ is von Neumann regular and that the canonical map $\psi_{M}$ : $M \rightarrow \mathcal{V}\left(Q_{K}(M)\right)$ is an isomorphism. Then $Q_{K}\left(M^{\prime}\right)$ is also von Neumann regular and the map $\psi_{M^{\prime}}: M^{\prime} \rightarrow \mathcal{V}\left(Q_{K}\left(M^{\prime}\right)\right)$ is an isomorphism.

Proof. Obviously we can assume that the order isomorphism $\varphi: A \rightarrow A^{\prime}$ respects the labelling of the arrows of the quivers $\mathcal{T}(A)$ and $\mathcal{T}\left(A^{\prime}\right)$ associated with $A$ and $A^{\prime}$ respectively; see Definition 2.1. Consequently we get a $K$-algebra isomorphism, denoted in the same way, $\varphi: Q_{K}(A) \rightarrow Q_{K}\left(A^{\prime}\right)$.

We have a natural isomorphism, still denoted by $\varphi$, from $e(A) Q_{K}(M) e(A)$ onto $e\left(A^{\prime}\right) Q_{K}(M) e\left(A^{\prime}\right)$ given by the composition of the isomorphisms

$$
e(A) Q_{K}(M) e(A) \longrightarrow Q_{K}(A) \longrightarrow Q_{K}\left(A^{\prime}\right) \longrightarrow e\left(A^{\prime}\right) Q_{K}(M) e\left(A^{\prime}\right),
$$

where the first and third maps are isomorphisms by Theorem 2.14. It follows that $I(A)$ and $I\left(A^{\prime}\right)$ are Morita equivalent (where $I(A)$ (resp. $I\left(A^{\prime}\right)$ ) denotes the ideal of $Q_{K}(M)$ generated by $e(A)$ (resp. $\left.e\left(A^{\prime}\right)\right)$ ). Write $Q:=Q_{K}(M)$. Take $Q_{1}=Q / I\left(A^{\prime}\right)$ and $Q_{2}=Q / I(A)$, and consider the $Q_{1}-Q_{2}$-bimodule $N=Q e(A) \otimes_{e(A) Q e(A)} e\left(A^{\prime}\right) Q$ and the $Q_{2}$ - $Q_{1}$-bimodule $M=Q e\left(A^{\prime}\right) \otimes_{e}\left(A^{\prime}\right) Q e\left(A^{\prime}\right) e(A) Q$, where $e(A) Q e(A)$ acts on $e\left(A^{\prime}\right) Q$ by $x \cdot y=\varphi(x) y$ for $x \in e(A) Q e(A)$ and $y \in e\left(A^{\prime}\right) Q$, and similarly for the action of $e\left(A^{\prime}\right) Q e\left(A^{\prime}\right)$ on $e(A) Q$. Let $U$ be the ring described in the proof of Proposition 4.5, so that $U$ is the ring of all matrices

$$
X=\left(\begin{array}{cc}
q+I\left(A^{\prime}\right) & n \\
m & q+I(A)
\end{array}\right)
$$

in $\left(\begin{array}{cc}Q_{1} & N \\ M & Q_{2}\end{array}\right)$. By Proposition 4.5 there is an injective unital $K$-algebra morphism $\omega: Q \rightarrow U$ given by $\omega(q)=\operatorname{diag}\left(q+I\left(A^{\prime}\right), q+I(A)\right)$. Moreover $U$ is von Neumann regular, and $\mathcal{V}(U) \cong M^{\prime}$ in a natural way, and we have an isomorphism

$$
Q /\left(I(A)+I\left(A^{\prime}\right)\right) \cong U / J
$$

where

$$
J=\left(\begin{array}{cc}
I(A) & N \\
M & I\left(A^{\prime}\right)
\end{array}\right)
$$

Set $e=1_{U}-\operatorname{diag}\left(0, e\left(A^{\prime}\right)\right) \in U$, and observe that $e$ is a full idempotent in $U$, that is, $U e U=U$. It follows that $\mathcal{V}(e U e) \cong M^{\prime}$. We are going to show that there is an isomorphism $\delta: Q_{K}\left(M^{\prime}\right) \rightarrow e U e$ such that the induced map

$$
\mathcal{V}\left(\delta^{-1}\right): M^{\prime} \cong \mathcal{V}(e U e) \rightarrow \mathcal{V}\left(Q_{K}\left(M^{\prime}\right)\right)
$$

is the canonical map $M^{\prime} \rightarrow \mathcal{V}\left(Q_{K}\left(M^{\prime}\right)\right)$. 
We define the map $\delta$ on the canonical generators of $Q_{K}\left(M^{\prime}\right)$, given in Definition 2.1. The reader can easily show that the defining relations are satisfied in $e U e$ and that the map $\mathcal{A} \rightarrow e U e$ so defined is $\left(\Sigma \cup \Sigma_{1}\right)$-inverting.

By Lemma 4.4, $\mathbb{P}^{\prime}:=\mathbb{P}\left(M^{\prime}\right)=\mathbb{P} \backslash A^{\prime}$, with the order relation induced by the order relation in $\mathbb{P}$ and the additional relations $q<p$ whenever $q \in A, p \in \mathbb{P}^{\prime} \backslash A$, and $\varphi(q)<p$ in $\mathbb{P}$. Define $\delta(e(p))=\omega(e(p)) \in U$ for $p \in \mathbb{P}^{\prime}$. Observe that $\left\{\omega(e(p)): p \in \mathbb{P}^{\prime}\right\}$ is an orthogonal family of idempotents in $e U e$ with sum $e$. Now for $q, p \in \mathbb{P}^{\prime}$ such that $q \in \mathrm{L}(\mathbb{P}, p)$, define $\delta(e(p, q))=\omega(e(p, q)), \delta\left(\alpha_{p, q}\right)=\omega\left(\alpha_{p, q}\right)$, and $\delta\left(\beta_{p, q}\right)=\omega\left(\beta_{p, q}\right)$. If $q \in A, p \in \mathbb{P}^{\prime} \backslash A$ and $\varphi(q) \in \mathrm{L}(\mathbb{P}, p)$, then define

$$
\begin{aligned}
& \delta(e(p, q))=\omega(e(p, \varphi(q)))=\left(\begin{array}{cc}
0 & 0 \\
0 & e(p, \varphi(q))
\end{array}\right), \\
& \delta\left(\alpha_{p, q}\right)=\omega\left(\alpha_{p, \varphi(q)}\right) \text {, and } \\
& \qquad\left(\beta_{p, q}\right)=\left(\begin{array}{cc}
0 & 0 \\
\beta_{p, \varphi(q)} \otimes e(q) & 0
\end{array}\right) .
\end{aligned}
$$

Note that this is well defined by our hypothesis that $\partial A \cap \partial A^{\prime}=\emptyset$.

Now it is straightforward to show that $\delta$ is an isomorphism. Indeed the induced map $Q_{K}\left(M^{\prime}\right) / I_{Q_{K}\left(M^{\prime}\right)}(A) \rightarrow$ eUe/eJe $=Q /\left(I(A)+I\left(A^{\prime}\right)\right)$ is an isomorphism by Remark 2.17, using our assumption that $\partial A \cap \partial A^{\prime}=\emptyset$. The restriction map $\bar{\delta}: I_{Q_{K}\left(M^{\prime}\right)}(A) \rightarrow e J e$ is also an isomorphism. For, note that elements in $Q_{K}\left(M^{\prime}\right)_{\left(\gamma_{1}, \gamma_{2}\right)}$ with $r\left(\gamma_{1}\right)=r\left(\gamma_{2}\right) \in A$ can be classified in four classes, depending on whether each $\gamma_{i}$ contains an arrow $(p, q)$ with $q \in A, p \in \mathbb{P}^{\prime} \backslash A$ and $\varphi(q) \in \mathrm{L}(\mathbb{P}, p)$, or does not contain it. Each of the four classes corresponds to a corner in

$$
\begin{aligned}
& e J e= \\
& \left(\begin{array}{cc}
I(A) & Q e(A) \otimes_{e(A) Q e(A)} e\left(A^{\prime}\right) Q\left(1-e\left(A^{\prime}\right)\right) \\
\left(1-e\left(A^{\prime}\right)\right) Q e\left(A^{\prime}\right) \otimes_{e\left(A^{\prime}\right) Q e\left(A^{\prime}\right)} e(A) Q & \left(1-e\left(A^{\prime}\right)\right) I\left(A^{\prime}\right)\left(1-e\left(A^{\prime}\right)\right)
\end{array}\right) .
\end{aligned}
$$

Thus, one obtains from Proposition 2.15 that $\bar{\delta}$ is surjective. In order to show injectivity of $\bar{\delta}$, take a nonzero element $x$ in $I_{Q_{K}\left(M^{\prime}\right)}(A)$. By Lemma 2.13, there exist $p \in A$ and $z_{1}, z_{2} \in Q_{K}\left(M^{\prime}\right)$ such that $z_{1} x z_{2}$ has the trivial pair of paths $(p, p)$ in the support, and all the other elements in the support of $z_{1} x z_{2}$ are of the form $\left(\gamma_{1}, \gamma_{2}\right)$, where $\gamma_{1}$ and $\gamma_{2}$ are paths in $\mathcal{T}(A)$ starting in $p$ and ending in a common vertex. Then we have that $\delta\left(z_{1} x z_{2}\right) \neq 0$, because the component in $Q_{K}\left(M^{\prime}\right)_{(p, p)}$ of $z_{1} x z_{2}$ is sent by $\delta$ to a nonzero diagonal element in $e J e$.

Therefore the map $\bar{\delta}$ is an isomorphism and consequently so is the map $\delta$, as desired.

\section{ACKNOWLEDGEMENTS}

The author is grateful to Ken Goodearl for his useful comments on a preliminary version of this paper, and to the anonymous referee for his or her very careful reading of the paper and stimulating suggestions.

\section{REFERENCES}

[1] G. Abrams, Morita equivalence for rings with local units. Comm. Algebra, 11 (1983), 801837. MR695890 (85b:16037)

[2] G. Abrams, G. Aranda Pino, The Leavitt path algebra of a graph, J. Algebra, 293 (2005), 319-334. MR2172342 (2007b:46085) 
[3] G. Abrams, G. Aranda Pino, Purely infinite simple Leavitt path algebras, J. Pure Appl. Algebra, 207 (2006), 553-563. MR 2265539 (2007f:16062)

[4] P. N. Ánh, L. Márki, Morita equivalence for rings without identity, Tsukuba J. Math., 11 (1987), 1-16. MR899719 (88h:16054)

[5] P. ARA, Extensions of exchange rings, J. Algebra, 197 (1997), 409-423. MR 1483771 (98j:16021)

[6] P. ARA, The realization problem for von Neumann regular rings, Proceedings of the Fifth China-Japan-Korea International Symposium on Ring Theory, Tokyo 2007, (eds. H. Marubayashi, K. Masaike, K. Oshiro, M. Sato); World Scientific, 2009, pp. 21-37.

[7] P. Ara, M. Brustenga, The regular algebra of a quiver, J. Algebra, 309 (2007), 207-235. MR2301238 (2008a:16019)

[8] P. Ara, M. Brustenga, Mixed quiver algebras, Preprint, arXiv:0909.0421v1 [math.RA].

[9] P. Ara, A. Facchini, Direct sum decompositions of modules, almost trace ideals, and pullbacks of monoids, Forum Math., 18 (2006), 365-389. MR2237927 (2007d:16012)

[10] P. Ara, K. R. Goodearl, E. Pardo, $K_{0}$ of purely infinite simple regular rings, $K$-Theory, 26 (2002), 69-100. MR.1918211 (2004e:19001)

[11] P. Ara, K. R. Goodearl, K. C. O'Meara, E. Pardo, Separative cancellation for projective modules over exchange rings, Israel J. Math., 105 (1998), 105-137. MR.1639739 (99g:16006)

[12] P. Ara, K. R. Goodearl, K. C. O'Meara, R. Raphael, $K_{1}$ of separative exchange rings and $C^{*}$-algebras with real rank zero, Pacific J. Math., 195 (2000), 261-275. MR 1782176 (2001m:46155)

[13] P. Ara, M. A. Moreno, E. Pardo, Nonstable $K$-theory for graph algebras, Algebras Represent. Theory, 10 (2007), 157-178. MR2310414(2008b:46094)

[14] P. Ara, F. Perera, F. Wehrung, Finitely generated antisymmetric graph monoids, J. Algebra, 320 (2008), 1963-1982. MR2437639

[15] G. M. Bergman, Modules over coproducts of rings, Trans. Amer. Math. Soc., 200 (1974), 1-32. MR0357502(50:9970)

[16] G. M. Bergman, Coproducts and some universal ring constructions, Trans. Amer. Math. Soc., 200 (1974), 33-88. MR0357503 (50:9971)

[17] G. M. Bergman, W. Dicks, Universal derivations and universal ring constructions, Pacific J. Math., 79 (1978), 293-337. MR.531320 (81b:16024)

[18] G. Brookfield, Cancellation in primely generated refinement monoids, Algebra Universalis, 46 (2001), 342-371. MR1857203 (2002g:20101)

[19] P. M. Cohn, "Free Rings and Their Relations", Second Edition, London Math. Soc. Monographs 19, Academic Press, London, 1985. MR800091 (87e:16006)

[20] K. R. Davidson, " $C^{*}$-algebras by example", Fields Institute Monographs, 6, American Mathematical Society, Providence, RI, 1996. MR1402012 (97i:46095)

[21] N. Dubrovin, P. Príhoda, G. Punisnki, Projective modules over the Gerasimov-Sakhaev counterexample, J. Algebra, 319 (2008), 3259-3279. MR2408317

[22] J. L. García, J.J. Simón, Morita equivalence for idempotent rings, J. Pure Appl. Algebra, 76 (1991), 39-56. MR1140639 (93b:16010)

[23] J. M. HowIE, "Fundamentals of semigroup theory", London Mathematical Society Monographs, New Series, 12, Oxford Science Publications. The Clarendon Press, Oxford University Press, New York, 1995. MR.1455373 (98e:20059)

[24] K. R. Goodearl, "Von Neumann Regular Rings", Pitman, London, 1979; Second Ed., Krieger, Malabar, FL, 1991. MR.533669 (80e:16011)

[25] K. R. Goodearl, "von Neumann regular rings and direct sum decomposition problems", Abelian groups and modules (Padova, 1994), Math. Appl. 343, 249-255, Kluwer Acad. Publ., Dordrecht, 1995. MR 1378203

[26] K. R. Goodearl, Leavitt path algebras and direct limits, Contemporary Mathematics 480 (2009), 165-187.

[27] N. Jacobson, Some remarks on one-sided inverses, Proc. Amer. Math. Soc., 1 (1950), 352355. MR0036223(12:75e)

[28] P. Menal, J. Moncasi, On regular rings with stable range 2, J. Pure Applied Algebra, 24 (1982), 25-40. MR647578 (83g:16025)

[29] J. Milnor, Introduction to algebraic $K$-theory, Princeton University Press, Princeton, 1971. MR0349811 (50:2304) 
[30] W. K. Nicholson, Lifting idempotents and exchange rings, Trans. Amer. Math. Soc., 229 (1977), 269-278. MR0439876 (55:12757)

[31] F. Perera, Lifting units modulo exchange ideals and $C^{*}$-algebras with real rank zero, $J$. Reine Angew. Math., 522 (2000), 51-62. MR.1758574 (2001g:46149)

[32] R. S. PierCe, "Countable Boolean Algebras", in Handbook of Boolean Algebras, Vol. 3, edited by J. D. Monk with R. Bonnet, Elsevier, 1989, 775-876. MR991610

[33] I. Raeburn, Graph algebras, CBMS Reg. Conf. Ser. Math., vol. 103, Amer. Math. Soc., Providence, RI, 2005. MR2135030 (2005k:46141)

[34] J. Rosenberg, "Algebraic $K$-Theory and Its Applications", Springer-Verlag, Graduate Texts in Math., 147, 1994. MR.1282290(95e:19001)

[35] A. H. Schofield, "Representation of Rings over Skew Fields", London Math. Soc. Lecture Note Series 92, Cambridge Univ. Press, Cambridge, UK, 1985. MR800853 (87c:16001)

[36] F. Wehrung, Non-measurability properties of interpolation vector spaces, Israel J. Math., 103 (1998), 177-206. MR:1613568 (99g:06023)

[37] F. Wehrung, The dimension monoid of a lattice, Algebra Universalis, 40 (1998), 247-411. MR1668068 (2000i:06014)

Departament de Matemàtiques, Universitat Autònoma de Barcelona, 08193, BelLATERra (BARCElona), SPAin

E-mail address: para@mat.uab.cat 